\title{
A continuously pumped reservoir of ultracold atoms
}

Der QUEST-Leibniz-Forschungsschule der Gottfried Wilhelm Leibniz Universität Hannover

zur Erlangung des Grades

Doktor der Naturwissenschaften

- Dr. rer. nat. -

genehmigte Dissertation von

Dipl.-Phys. Jan Mahnke, geboren am 29.05.1984 in Hannover 
Referent:

Prof. Dr. Wolfgang Ertmer,

Institut für Quantenoptik, Hannover

Korreferent: $\quad$ PD Dr. Carsten Klempt,

Institut für Quantenoptik, Hannover

Tag der Promotion: 12. Oktober 2015 


\section{Zusammenfassung}

In den letzten Jahrzenten wurde der experimentelle Zugang zum Quantenregime in einer Vielzahl von Systemen erreicht, unter anderem in dünnen, ultrakalten Gasen, gefangenen Ionen, mikromechanischen Oszillatoren, und supraleitenden Schaltkreisen. Diese Systeme ermöglichen spannende Anwendungen wie Quantencomputer, Quantenkommunikation, Quantenmetrologie und Quantensimulation. Die quantenmechanischen Besonderheiten eines physikalischen Systems treten erst durch Kühlung zur Tage, wenn thermische Anregungen vernachlässigt werden können.

Bislang werden die kältesten Temperaturen in ultrakalten Gasen durch die Kombination von Laserkühlung mit Evaporation erreicht, was inhärent zur Produktion einzelner Ensembles führt. Viele Anwendungen könnten jedoch von einer kontinuierlichen Quelle ultrakalter Atome profitieren, zum Beispiel das sympathetische Kühlen von Molekülen oder nanoskopischen Objekten. Idealerweise wird diese Quelle ohne Licht betrieben, da die meisten Moleküle viele interne Zustände besitzen und somit Licht vieler Wellenlängen absorbieren können. Eine weitere vielversprechende Anwendung kontinuierlicher Quellen ultrakalter Materie ist die Atominterferometrie. Diese Interferometer messen inertiale Kräfte, Zeit und Gravitation. Derzeit wird jedes Ensemble bei der Auswertung zerstört und die Messung muss unterbrochen werden um ein weiteres zu erzeugen. Kontinuierliche Quellen können dazu beitragen diese Limitierung aufzuheben.

Im Mikrokelvin-Bereich existiert bislang keine kontinuierliche Quelle. Diese Arbeit präsentiert die erste Präparation einer solchen ultrakalten Atomwolke, die kontinuierlich nachgeladen wird und vor Licht geschützt ist. Das kontinuierliche Reservoir wird erzeugt indem ein magnetisch geführter Atomstrahl in ein längliches, magnetisches Fallenpotenzial geladen wird. Dieses statische Potenzial hat eine Eingangsbarriere, die von den Atomen überwunden werden kann. In der Falle stoßen die Atome miteinander und verteilen ihre kinetische Energie um. Atome, die Energie hinzugewinnen können die Falle verlassen, aber Atome, die Energie verlieren werden gefangen. Ein Zustand mit $3.8 \times 10^{7}$ Atomen bei einer Temperatur von $102 \mu \mathrm{K}$ wird erreicht und aufrechterhalten. Der Aufbau des kontinuierlichen Ladeverfahrens wird in dieser Arbeit beschrieben. Ein Schlüsselelement ist ein mesoskopischer Atomchip, der alle Magnetfelder für das kontinuierliche Ladeverfahren bereitstellt. Die anfängliche Atomquelle ist ein zweistufiges, magnetooptisches Fallensystem mit hohem Fluss. Aus den vorgekühlten Atomen wird ein Atomstrahl erzeugt und durch eine Streulichtabschirmung zu der statischen Falle geführt.

Das Kühlverfahren stellt keine besonderen Anforderungen an die atomaren Eigenschaften und kann somit auf eine breite Auswahl von Teilchen übertragen werden. Die Resultate bieten gute Perspektiven für die Anwendung als kontinuierliche Quelle ultrakalter Atome, entweder als Kühlmedium zum sympathetischen Kühlen oder zur Verwendung in der Metrologie.

Schlagwörter: ultrakalte Quantengase, kontinuierliche Quellen, mesoskopischer Atomchip 


\section{Abstract}

In the last decades, experimental access to the quantum regime has been gained in a large variety of systems, including dilute ultracold gases, trapped ions, micromechanical oscillators, superconducting circuits, and many more. These systems allow for such exciting applications as quantum computing and communication, quantum metrology and quantum simulation. A physical system's quantum mechanical features can only be revealed by cooling it until the thermal excitations are negligible.

To date, the coldest temperatures are reached with ultracold gases by a combination of laser cooling and evaporation, inherently producing individual ensembles. However, many applications can greatly benefit from a continuous source of ultracold atoms, for example sympathetic cooling of molecules or nanoscopic objects. Ideally, this cooling source operates without light, as most molecules have a rich internal structure and can absorb photons of many wavelengths. Another promising application of continuous sources of ultracold matter is atom interferometry. Such interferometers perform measurements of inertial forces, time and gravity. Currently, each cold sample is destroyed by its evaluation and the measurement is interrupted by the preparation of a new samples. Continuous sources can help to eliminate this restriction. So far, no dark and continuous sources exist in the microkelvin regime.

This thesis presents the first preparation of such an ultracold atom cloud which is continuously replenished and protected from light. The continuously refilled reservoir is prepared by loading a magnetically guided atom beam into an elongated magnetic trapping potential. This static potential has an entrance barrier which allows atoms from a guided beam to enter the trap. Once in the trap, the atoms undergo collisions and redistribute their kinetic energy. Those gaining energy can escape again, but those losing energy are trapped. A steady state with $3.8 \times 10^{7}$ atoms atoms at a temperature of $102 \mu \mathrm{K}$ is reached and maintained. The setup of the continuous loading scheme is described in this thesis. A key component is a mesoscopic atom chip which produces all magnetic fields for the continuous loading scheme. The initial atom source is a high-flux double magneto-optical trap system. An atomic beam is created out of the precooled atoms and guided to the static trap through a stray light protection allowing for a dark retrapping region.

The cooling scheme does not rely on any specific atomic properties of the atoms and can thus be applied to a wide range of particles. The results offer exciting perspectives for an application as a continuous source of ultracold atoms, either as a coolant for sympathetic cooling or as a resource for metrology.

Keywords: ultracold quantum gases, continuous sources, mesoscopic atom chip 


\section{Contents}

1. Introduction to dark and continuous cooling 1

1.1. Introduction . . . . . . . . . . . . . . . . . . . . 1

1.2. Temperature and cooling . . . . . . . . . . . . . 2

1.3. Dark cooling. . . . . . . . . . . . . . . . 7

1.3.1. Buffer gas cooling . . . . . . . . . . . . . 7

1.4. Continuous cooling . . . . . . . . . . . . . . 8

1.4.1. Zeeman slowers . . . . . . . . . . . . . . 8

1.4.2. Stark decelerator . . . . . . . . . . . . . . . . . 10

1.4.3. Two-dimensional magneto-optical trap . . . . . . . . . . 11

1.4.4. Sympathetic cooling of molecular ions . . . . . . . . . . . . 12

1.5. Continuous and dark cooling . . . . . . . . . . . . . 13

1.5.1. ${ }^{3} \mathrm{He}-{ }^{4} \mathrm{He}$ dilution refrigerator . . . . . . . . . . . . . . 13

1.6. Summary . . . . . . . . . . . . . . . . . . . . 14

2. Experimental apparatus $\quad \mathbf{1 5}$

2.1. Experimental concept . . . . . . . . . . . . . . . . 15

2.2. Vacuum system . . . . . . . . . . . . . . . . . . 17

2.3. Rubidium laser system . . . . . . . . . . . . . . . . . . . . 19

2.4. Electronics and current control . . . . . . . . . . . . . . . . . 21

2.4.1. Current control with MOSFET circuits . . . . . . . . . 22

2.4.2. Analog PI controller for the chip currents . . . . . . . . . . . 23

2.5. Computer-based experimental control . . . . . . . . . . . . 24

2.5.1. Experimental control hardware . . . . . . . . . . . . 24

2.5.2. Experimental control software . . . . . . . . . . . . 25

2.5.3. Automated experimental optimization . . . . . . . . . 25

2.6. Atom chip traps . . . . . . . . . . . . . . . . . . . . 27

2.6.1. Development of atom chip traps . . . . . . . . . . . 27

2.6.2. Magnetic trapping . . . . . . . . . . . . . . 28

2.6.3. Principles of atom chip traps . . . . . . . . . . . . . . . 29

2.7. Mesoscopic atom chip . . . . . . . . . . . . . . . . . . 33

2.8. Double MOT system . . . . . . . . . . . . . . . 35

2.9. Molasses and state preparation . . . . . . . . . . . . 37

3. Continuously pumped reservoir of ultracold atoms 41

3.1. Preparation of the atomic beam . . . . . . . . . . . . . . . . 43 


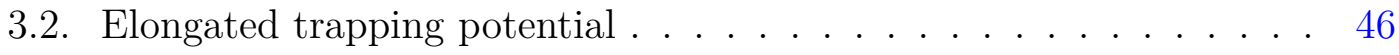

3.3. Continuous loading . . . . . . . . . . . . . . . . . . 48

3.4. Trap enhancement with a dipole beam . . . . . . . . . . . . 52

4. Microwave sidebands for laser cooling by direct modulation of a tapered amplifier

4.1. Compact laser systems . . . . . . . . . . . . . . . . . 55

4.2. Modulating a tapered amplifier . . . . . . . . . . . 56

4.2.1. Tapered amplifier introduction . . . . . . . . . . . . 56

4.2.2. Tapered amplifier setup . . . . . . . . . . . . . . . 57

4.2.3. Microwave source for the modulation of the supply current . 59

4.2.4. Modulation setup . . . . . . . . . . . . . . . . 60

4.3. Implementation in an experimental environment . . . . . . . . 62

4.4. Characterization of the sidebands using a magneto-optical trap . . . 65

4.5. Conclusion . . . . . . . . . . . . . . . . . 67

$\begin{array}{ll}\text { 5. Outlook } & 69\end{array}$

$\begin{array}{ll}\text { A. Vacuum components } & 73\end{array}$

B. Rubidium $87 \mathrm{D}_{2}$ transition hyperfine structure $\quad 75$

$\begin{array}{ll}\text { C. Tapered amplifier housing } & 77\end{array}$

$\begin{array}{ll}\text { Bibliography } & 81\end{array}$

$\begin{array}{ll}\text { Publications } & 93\end{array}$ 


\section{Introduction to dark and continuous cooling}

\subsection{Introduction}

Laser cooling has sparked the field of cold atom optics and has enabled the early deceleration and stopping of atomic beams in 1982 [1-3] and the development of the magneto-optical trap in 1987 [4-6] as well as modern atom experiments capable of producing Bose-Einstein condensates [7-9] at a $1 \mathrm{~Hz}$ rate [10]. The diverse applications put increasingly specialized requirements on sources of cold particles besides temperature, number of particles and density. The work presented in this thesis offers intriguing benefits, a truly continuous ultracold sample which is completely shielded from any light sources. Two distinct applications were especially motivating to set these goals: Atom interferometry and sympathetic cooling of molecules.

Atom interferometry is an active field of research which has lead to the development of measurement devices with unprecedented precision for rotation [11-13], acceleration [14,15], gravity [16], gravity gradients [17,18] and time [19-21]. Even proposals for the detection of gravitational waves by atom interferometry are discussed [22-25]. All of these interferometry schemes share that they prepare a cold sample, perform a measurement destroying the sample and start over. For these experiments, truly continuous sources of ultracold particles are highly desirable. In particular, optical clocks - the most precise frequency standards to date - are currently limited by the Dick effect [26], which could be avoided by a continuous interrogation scheme. Similar restrictions exist for inertial sensors based on atom interferometry.

Another field of research that would benefit from a continuous and dark reservoir of ultracold atoms is the sympathetic cooling of molecules. Compared to atoms, molecules have a rich internal structure which opens interesting opportunities for tests of fundamental constants [27], quantum chemistry [28] and the development of quantum computers [29]. Even though in high demand, the preparation of cold molecules is still a difficult task. The rich internal structure of molecules allows very rarely for effective cooling transitions and thus laser cooling is impossible for most molecules. One promising way to cool a wide range of molecules is the use of sympathetic cooling. Here, the molecules are brought into contact with a pre-cooled cloud of atoms and is thus cooled sympathetically through collisions. 
The problem with most cold atom sources today is that most of them employ laser light for cooling. Unfortunately, the internal structure of most molecules is rich enough, so that almost every light frequency can be absorbed and efficient cooling is hindered. To avoid this, a cooling agent without laser light is needed. This application also highly benefits from a continuous source, which greatly increases the cooling time and allows for cold temperatures even for not optimal collision properties.

In this thesis, a continuously replenished reservoir of ultracold atoms is produced in the microkelvin regime. The experiment is based on a proposal by the group of D. Guery-Odelin and J. Dalibard [30]. The core idea is to trap atoms from a guided atomic beam in a static and conservative magnetic trap. This trap consists of a highly elongated potential with an entrance barrier. Incoming atoms pass this barrier and undergo collisions within the trap. These collisions result in a redistribution of kinetic energy. Those atoms gaining energy can leave the trap, while atoms losing energy are trapped. due to a continuous beam, the reservoir of ultracold atoms is continuously replenished and can serve as a permanent source of ultracold atoms. The magnetic fields for the trap and guide are completely created by a mesoscopic atom chip and no laser light is needed for the accumulation process.

The thesis is structured as follows: This chapter gives an introduction to temperature and cooling in general as well as continuous and dark cooling. Subsequently, several experiments of the field are presented to show the current state and the relation to this work. Chapter 2 describes the main components of the apparatus used to create the reservoir. It shows the vacuum and the laser system, as well as the current control for the atom chip and the computer based experimental control. The mesoscopic atom chip is introduced after a general introduction to atom chip traps. Finally, the double magneto-optical trap setup and the following state preparation is described. In Chapter 3, the characterization and loading process of the static trap is described, starting with the preparation of the atomic beam and the creation of the elongated trapping potential. The continuous loading process and enhancements are presented. Last, Chapter 4 describes an extension of the laser system that eliminated the need for one of the two master lasers by a microwave modulation of a tapered amplifier. The creation of microwave sidebands by the modulation of the tapered amplifier current is presented. The performance is evaluated and the system is applied to operate the magneto-optical trap.

\subsection{Temperature and cooling}

Temperature affects many aspects from daily life to science and technology. Life itself is highly dependent on temperature, because the rate of many biological processes is related to it. Even though temperature plays a vital role in daily life, the temperature range we can naturally experience is within a few $10^{\circ} \mathrm{C}$. The control of high temperature was a major driving force in the development of the modern 
world: From the control of combustion and steam with major advancements during industrialization times to modern breakthroughs in science and technology. Exploration of the high-energy scale lead to plasmas, nuclear fission and fundamental research in particle accelerators. Although younger, low-energy physics is no less exciting. Many interesting phenomena can be observed when exploring the cold, like superconducting materials with zero electrical resistance, superfluids without viscosity as first seen in liquid helium or the possibility to study quantum physics in macroscopic structures. A range of temperatures for comparison is given in Fig. 1.1.

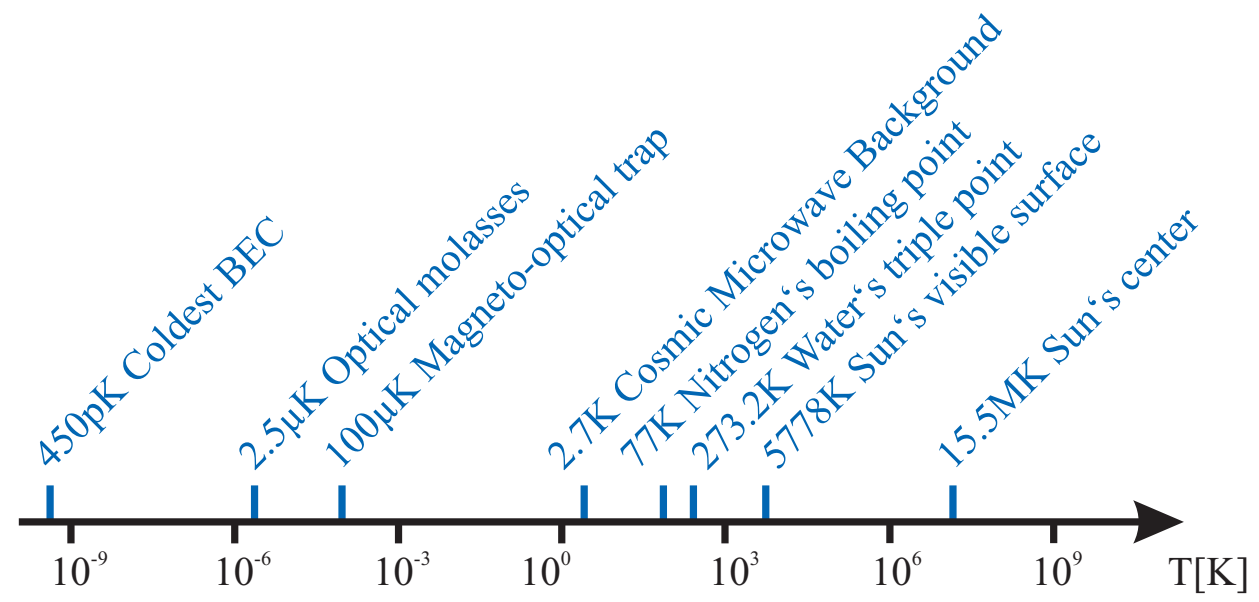

Figure 1.1.: Examples of temperature. Some absolute temperatures to scale, including the coldest BEC [31], optical molasses [32], cosmic microwave background radiation [33] and temperatures of the sun's surface and center [34]. On this logarithmic scale, the temperature of water's triple point is "closer" to the sun's core temperature than to the coldest temperatures achieved in the laboratory.

The vast effects of temperature makes it important to exactly determine its value. Over the time, a number of methods were developed to measure the temperature over the entire range. Many physical properties are exploited for the measurements. One example is the change of geometrical properties, as most bodies expand when temperature increases. This is used in thermometers based on liquid mercury or alcohol or in bimetallic strips. The thermoelectric effect is utilized in the widespread thermocouples, that can measure over a wide temperature range depending on the alloy combination used. Crystal oscillators change their oscillation frequency with the temperature and can convert a temperature measurement in a highly sensitive frequency measurement. The thermal radiation of a given object changes characteristically with the temperature and is especially well suited to measure hot bodies with non-contacting pyrometers. 
Given the measuring applications in very different temperature regimes, many temperature units with different calibration points came into use and are in part still used today. Except for the United States, which use the Fahrenheit scale $\left({ }^{\circ} \mathrm{F}\right)$, most countries today use the Celsius scale $\left({ }^{\circ} \mathrm{C}\right)$ for most of the common temperature measurements. The Celsius scale was originally defined by the freezing point of water at $0{ }^{\circ} \mathrm{C}$ and the boiling point of water at $100^{\circ} \mathrm{C}$, both at sea level pressure. Since 1954, the Celsius scale is defined by two different points and also directly fixed to the Kelvin scale (K): The lowest temperature possible, absolute zero, is defined as $-273.15^{\circ} \mathrm{C}$ and $0 \mathrm{~K}$ and the reproducible triple point of specially purified water as $0.01{ }^{\circ} \mathrm{C}$ and $273.16 \mathrm{~K}$. The Kelvin is the SI base unit for thermodynamic temperature [35] and widely used in scientific applications.

An illustrative physical description of the temperature can be given for dilute gases by the kinetic theory of gases. It assumes an ideal gas, which is comprised of small particles that only undergo elastic collisions with themselves or the wall of their container. It also assumes that there is no interaction among the particles other than direct collisions. Under these conditions, there is a simple relation between the macroscopic quantity temperature $T$ and the microscopic property of the particles, the mean kinetic energy $\bar{E}_{k i n}$. It is given by [36]

$$
\bar{E}_{k i n}=\frac{m}{2} \overline{v^{2}}=\frac{3}{2} k_{B} T
$$

with the Boltzmann constant $k_{B}=1.3806488(13) \times 10^{-23} \mathrm{~J} / \mathrm{K}$ [37]. This description is particularly well fitting for atomic gases in three dimensions but gets inaccurate for molecular gases with their internal degrees of freedom, like rotation and vibration. A more general description states that the mean kinetic energy of an atom at a temperature $T$ is given by

$$
\bar{E}_{k i n}=\frac{1}{2} k_{B} T \text { per degree of freedom. }
$$

Equation (1.1) sets a lower bound on the temperature. Atoms at rest would have the lowest temperature possible, absolute zero at $0 \mathrm{~K}$. The temperature can get extremely close to $0 \mathrm{~K}$ with modern cooling methods. To understand cooling, the reduction of mean energy, one has to take the distribution of energy into account. Equation (1.1) relates the mean kinetic energy to the temperature, but it does not give any information about the distribution of the energy among the particles. The distribution of the velocity among the particles in an ideal gas at thermal equilibrium can be described by the Maxwell-Boltzmann distribution [36]

$$
f(v)=\left(\frac{m}{2 \pi k_{B} T}\right)^{3 / 2} \cdot 4 \pi v^{2} \cdot e^{-m v^{2} / 2 k_{B} T} .
$$

This characteristic probability distribution gives the probability to find a particle of the ensemble at a given velocity. As an illustration, Fig. 1.2 shows the 


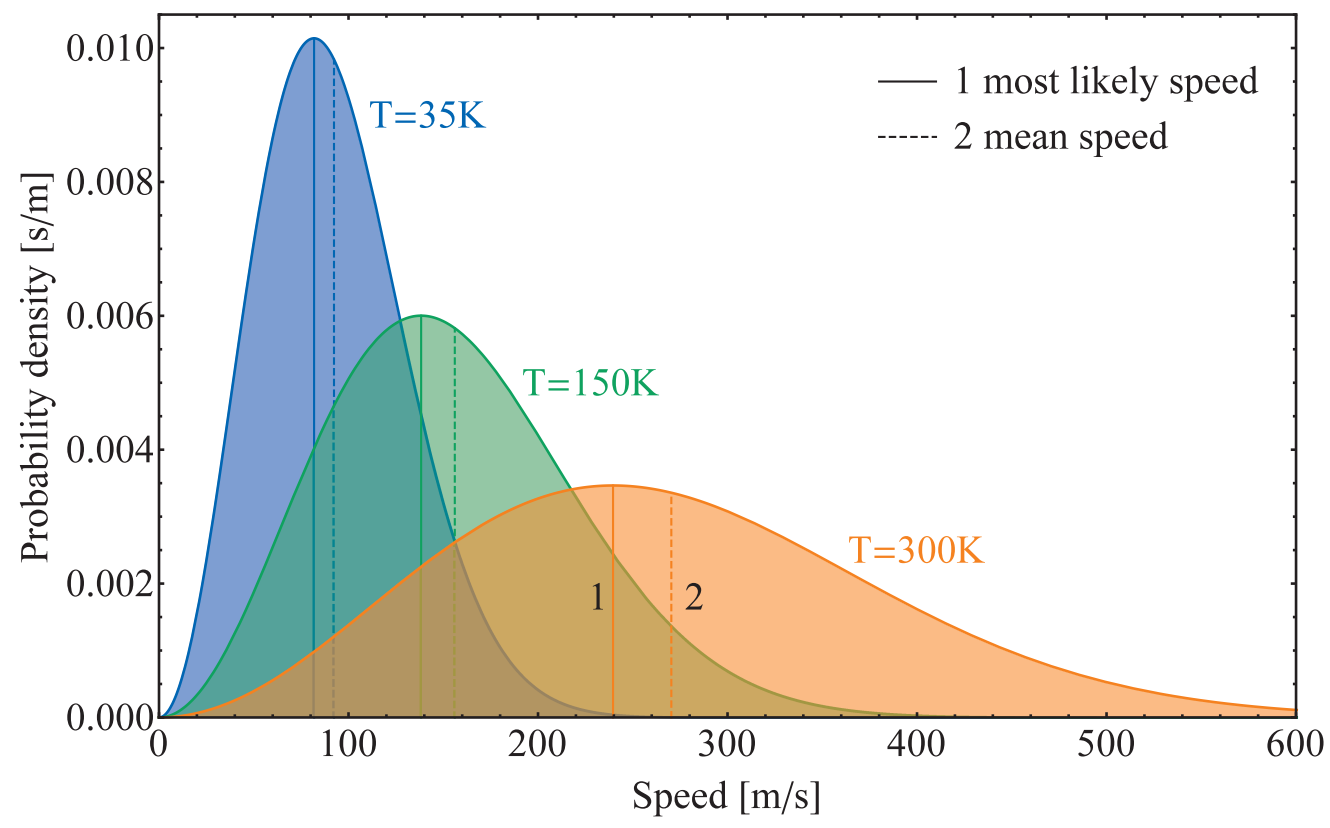

Figure 1.2.: Maxwell-Boltzmann distribution. Three distributions for an idealized gas with a particle mass equal to ${ }^{87} \mathrm{Rb}$ at $300 \mathrm{~K}, 150 \mathrm{~K}$ and $35 \mathrm{~K}$. The most likely and mean speed are given by a solid and dashed line.

Maxwell-Boltzmann distribution for an idealized gas of particles with the mass of ${ }^{87} \mathrm{Rb}$ at three different temperatures with the most likely and the mean velocity. Cooling techniques have to take into account the inhomogeneous distribution of energy among the particles. For example, evaporative cooling techniques rely on the removal of particles with well above average energies. Evaporative cooling, in combination with other modern techniques like laser cooling, can decrease the temperature into the ultracold regime.

In many cases, as temperature drops into the nanokelvin regime, the quantum nature of dilute gases becomes apparent. At such low temperatures, bosonic gases like ${ }^{87} \mathrm{Rb}$ vapor enter a phase called the Bose-Einstein condensate (short: BEC), where the quantum nature dominates (see Fig. 1.3). When still at high temperatures, dilute gases can be described as ideal gases and the particles behave similar to billiard balls undergoing elastic collisions. At low temperatures, the quantummechanical wave-particle duality becomes relevant and the particles have to be described as wave packets with an extent on the order of the thermal de Broglie wavelength [38]

$$
\lambda_{d B}=\sqrt{\frac{2 \pi \hbar^{2}}{m k_{B} T}} .
$$

Below the critical temperature $T_{c}$, the size of the wave packets becomes compa- 

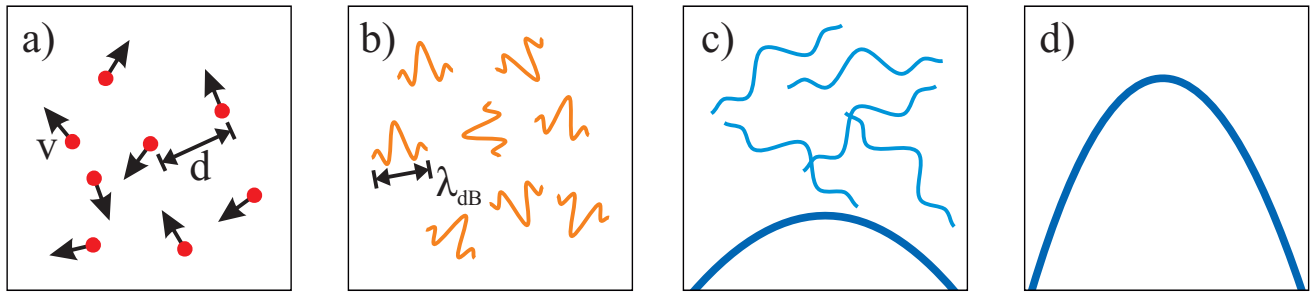

Figure 1.3.: Bose-Einstein condensation. a) High temperature: Classical description as an idealized gas. b) Low temperature: Behavior of wave packets with extension $\lambda_{d B}$. c) Temperature reaches critical temperature: $\lambda_{d B}$ comparable to distance between atoms and condensation starts. d) Temperature approaches zero: Pure Bose-Einstein condensate. The image is based on [38].

rable to the mean distance between the particles and they start to overlap. The indistinguishable bosons undergo a phase transition and enter the quantum degenerate regime where atoms coherently occupy the same quantum mechanical state, forming the Bose-Einstein condensate. A good measure to quantify the macroscopic occupation is the phase-space density $\mathcal{D}$, that states the number of atoms in a cube with edge length of $\lambda_{d B}[38]$

$$
\mathcal{D}=n \cdot \lambda_{d B}^{3}
$$

with the spatial density $n$. When cooling into the quantum degenerate regime, the phase-space density presents a better indicator for the efficiency of the cooling process than the consideration of the temperature. For example, the adiabatic expansion of an atomic gas can decrease its temperature, but the density also decreases and phase-space density is preserved.

The Bose-Einstein condensate was already predicted in 1925 by A. Einstein [39], based on the work by S. N. Bose on the statistical description of photons [40]. However, the advanced description of interactions in real matter proved to be a challenge. The discovery of superfluid features of liquid helium [41, 42] in 1937 was a cornerstone in the further description of Bose-Einstein condensates by F. London [43] and O. Penrose and L. Onsager [44]. The strong interaction within a liquid of superfluid helium-4 allowed only a small fraction of the atoms to condense and thus require a more advanced description of the condensation process. At this point, a major goal was to show Bose-Einstein condensation in dilute gases.

Along the way, a number of important steps had to be taken. The development of the laser around $1960[45,46]$ was a prerequisite for laser cooling of atoms. Starting in 1982, the deceleration and stopping of atomic beams was achieved [13]. In 1986, the slowing and trapping of atoms in a three-dimensional magnetooptical trap was proposed [47] and realized in 1987 [48]. The proposal [49,50] and execution [51] of evaporative cooling, first with atomic hydrogen in 1988, presented 
the last necessary ingredients for the long-awaited experimental realization of the Bose-Einstein condensation of rubidium, sodium and lithium in 1995 [7-9].

These dilute clouds of condensed, bosonic alkali atoms are weakly interacting and can therefore often be described with a mean-field theory. This description dates back on an approach made by N. Bogolubov [52] in 1947 and states that all the particles in the condensate can be described by a common wave function. The time evolution of such a condensate wave function is given by a nonlinear Schrödinger equation, the Gross-Pitaevskii equation [53,54]. For typical experimental conditions, this condensate wave functions can reach an extent on the order of hundred micrometers, enabling a direct detection of the density distribution through absorption imaging. This unique feature allows for the direct observation of quantum phenomena on macroscopic length scales and is one reason for the huge interest in the field of ultracold gases.

\subsection{Dark cooling}

In dark cooling schemes, a cold ensemble is created which does not suffer from any light. This type of cooling is rather seldom due to the ubiquitous laser cooling of atoms and the widespread optical dipole traps. Even a low-power optical pumping beam, as used in an atomic diode [55], provide and irradiation with potentially unwanted effects. In this section, buffer gas cooling is presented as an example of a dark cooling scheme. If desired, this cooling scheme could also become a continuous scheme with the right choice of a buffer gas supply.

\subsubsection{Buffer gas cooling}

Buffer gas cooling employs a cold gas to cool other atoms or molecules through thermalization when brought into contact. Buffer gas cooling was one of the earliest cooling schemes that reached millikelvin temperatures without the use of laser light. In 1995, J.M. Doyle and colleagues presented a possible experimental setup that used ${ }^{3} \mathrm{He}$ as a buffer gas, cooling paramagnetic atoms or molecules [56]. For $\mathrm{O}_{2}$ molecules, they calculated a thermalization temperature of $240 \mathrm{mK}$, cold enough to be loaded into a magnetic trap. After the loading process, the buffer gas could be removed and evaporative cooling could be used to further increase the phase-space density.

In 2009, S.C. Doret, C.B. Connolly, W. Ketterle and J.M. Doyle presented an experiment which used solely buffer gas cooling and evaporative cooling to reach Bose-Einstein condensation [57] in ${ }^{4} \mathrm{He}^{*}$. They employ a dilution refrigerator (see Chap. 1.5.1) to maintain a plastic container at $200 \mathrm{mK}$ and load it with a gas of ${ }^{4} \mathrm{He}$. A small fraction of the gas is converted to ${ }^{4} \mathrm{He}^{*}$ with an $\mathrm{rf}$ discharge. The ${ }^{4} \mathrm{He}^{*}$ is trapped and cooled to $500 \mathrm{mK}$ by collisions with the buffer gas. Afterwards, the buffer gas is removed. A series of compression and evaporation phases lead 
to a temperature reduction and, at about $5 \mu \mathrm{K}$, a Bose-Einstein condensate forms with $2 \times 10^{6}$ atoms. These buffer gas cooling schemes have reached the millikelvin regime and combined with evaporative cooling, they are among the coldest samples produced without the application of laser light.

\subsection{Continuous cooling}

A continuous cooling scheme is a process that could in principle run indefinitely. In reality, these schemes often have technical reasons that limit the cooling time, for example cool off time for heat dissipation. This chapter presents several continuous cooling schemes, including wide-spread atomic beam sources: Zeeman slowers, Stark decelerators and two-dimensional magneto-optical traps. Sympathetic cooling can also be continuous when a bath is replenished. This is true for the cooling scheme for molecular ions by E.R. Hudson and colleagues which is presented in this section. This cooling scheme can potentially also be a dark cooling scheme, when a dark heat bath is chosen and the trapping potentials can be realized without laser light.

\subsubsection{Zeeman slowers}

Zeeman slowers constitute an important step to produce neutral atomic beams that are slow enough to be trapped. They work by illuminating an atomic beam with a counter-propagating laser beam. The atoms scatter photons from this laser beam when it is resonant to an atomic transition. Because the laser beam is counterpropagating, the scattered photons transfers a mean momentum to the atoms that reduces their velocity. This works as long as the Doppler shift from the moving atoms is canceled by an appropriate light shift. However, when the atoms slow down the Doppler shift reduces and the atoms shift out of resonance quickly. So, although deflection of atoms by resonant light was demonstrated by R. Frisch already in 1933 [58] it took until 1982 when laser development enabled W. D. Phillips and $\mathrm{H}$. Metcalf to demonstrate a method to compensate the varying Doppler shift and to employ laser light to decelerate an atomic beam considerably [1].

In their experiment, W. D. Phillips and H. Metcalf show that a spatially varying magnetic field can introduce a tailored Zeeman shift which is able to compensate Doppler shift of the decelerating atoms. As a source, they use an oven to create a collimated supersonic sodium beam. This beam is directed through a 60-cm long solenoid with a decreasing amount of windings over its length, which is used to create the desired decreasing magnetic field. This brings the atoms into constant resonance with the fixed-frequency laser.

As an estimate of the photons needed in the cooling process they consider cooling with a frequency $\nu$ on the $3 S$ to $3 P$ transition of sodium. Cooling on this line changes the velocity of the sodium atoms by scattering $n$ photons by 


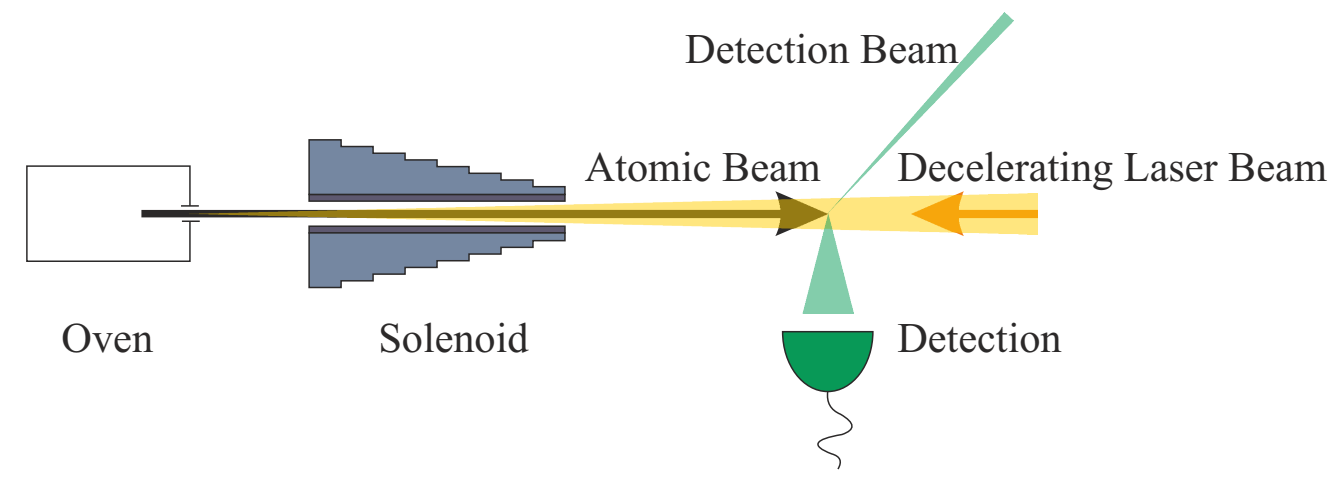

Figure 1.4.: Typical Zeeman slower setup. A hot, low-divergence atomic beam originates from an oven. It is overlayed with a counter-propagating decelerating laser beam focused at the nozzle of the oven. To continuously shift the atoms into resonance, they pass a solenoid. It produces a spatially varying magnetic field with a varying Zeeman shift to correct for the Doppler shift of the decelerating atoms. The length of the solenoid ranges from several ten centimeters to meters. After passing of the solenoid, the beam can either be analyzed with a detection or used for further experiments.

$n h \nu / M c \approx 3 n \mathrm{~cm} / \mathrm{s}$, with the mass of a sodium atom $M$, the speed of light $c$ and the Planck constant $h$. The $3 S-3 P$ transition of sodium has a lifetime of $16 \mathrm{~ns}$ and a saturation intensity of $6 \mathrm{~mW} / \mathrm{cm}^{2}$ [59]. When using large intensities to saturate the transition, atoms can spend half the time in the $3 P$ state and experience the maximal deceleration of approximately $10^{8} \mathrm{~cm} / \mathrm{s}^{2}$. For atoms with a speed of $v_{0}=10^{5} \mathrm{~cm} / \mathrm{s}$ to be stopped, it takes about $1 \mathrm{~ms}$ over a $50 \mathrm{~cm}$ path to scatter roughly 30000 photons [1]. In the first Zeeman slower, W. D. Phillips and H. Metcalf managed to scatter about 15000 photons and saw a velocity change of about $40 \%$.

Today, Zeeman slower present a versatile and important tool to slow down many different species. For example xenon, lithium and ytterbium can be cooled and trapped in a magneto-optical trap [60,61]. Even though radial beam divergence makes it difficult to stop beams completely, this has been demonstrated [62]. The driven coils of the Zeeman slower may cause problems in some experiments, as they increase the overall power consumption and can introduce noise. This is circumvented by a Zeeman slower constructed out of permanent magnets [63].

Instead of varying the magnetic field, the frequency of the laser light can be tuned in order to compensate for the changing Doppler shift. Although deceleration of $\mathrm{Na}$ atoms has been demonstrated with this technique [64], it is more complex to operate as it requires a dynamical control. Thereby, it also does not allow for a continuous deceleration. 


\subsubsection{Stark decelerator}

Stark decelerators have proven to be very successful at creating sub-Kelvin matter beams. They are particularly important in producing cold dipolar molecules. Their basic working principle is depicted in Fig. 1.5. They exploit that molecules with an electric dipole moment gain Stark energy upon entering an electric field. Such an electric field can be provided by two rods perpendicular to the beam axes typically charged with voltages in the $\pm 10 \mathrm{kV}$-range. As the molecules would again lose the Stark energy and thereby regain the kinetic energy when leaving the field, a time dependent solution is necessary. Upon reaching the potential maximum, the rods are quickly switched off and the next pair is charged. Thus, the molecules do not regain the lost kinetic energy and face again a potential hill. In this way, the molecules lose a fraction of their kinetic energy when passing a single pair of rods. The deceleration can be increased dramatically by operating several stages in series. Typically the number of stages is in the hundreds. Due to the time dependence, the overall process produces a pulsed slow beam.

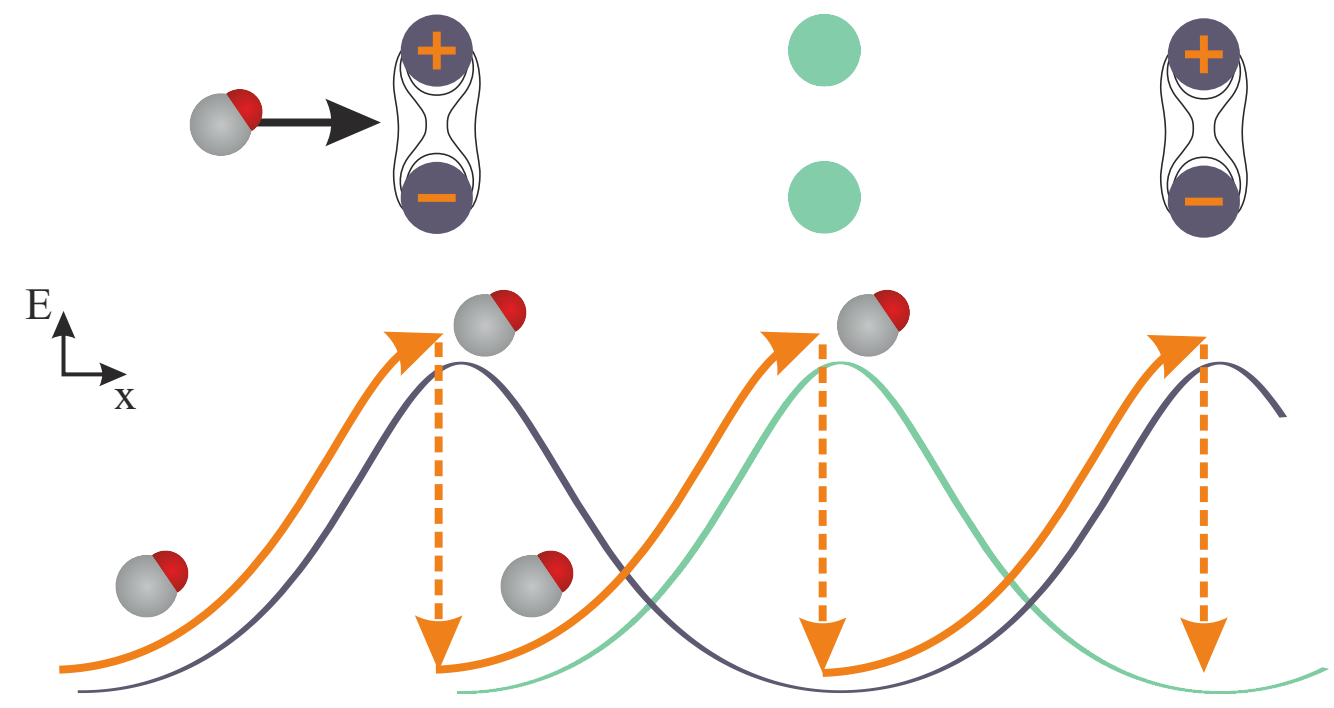

Figure 1.5.: Molecules passing a Stark decelerator. Top: Molecules passing through a series of pulsed electric field rods. Bottom: The corresponding electric field potential. First, the molecules in a low-field seeking climb the blue potential hill. The electric field is quickly switched when the molecules reach the center of a stage. The gained Stark energy upon climbing the potential hill is lost and the molecules are back at the bottom of a potential hill. By passing a chain of stages, the molecules steadily decelerate.

In 1999, H. Bethlem, G. Berden and G. Meijer were the first to successfully operate a Stark decelerator [65]. They worked with a molecular beam machine, 
producing a pulsed CO beam with a velocity of $230 \mathrm{~m} / \mathrm{s}$. They used direct laser excitation of the ground state to prepare the atoms in the first electronically excited state. This leads to a relatively large dipole moment of 1.37 Debye and a linear Stark dependency in an electrical field. The molecules pass a skimmer and a pulsed hexapole acting as a lens before they enter a $35 \mathrm{~cm}$ long Stark decelerator. 63 equidistant electric field stages consisting out of two parallel rods each are charged with Voltages of up to $\pm 10 \mathrm{kV}$. This generates electric fields with a maximum of $100 \mathrm{kV} / \mathrm{cm}$. The molecules with a velocity fitting to the phase of the sequential switching of the electric fields lose kinetic energy every stage. Time of flight imaging could show that CO molecules entering with $225 \mathrm{~m} / \mathrm{s}$ were slowed down to $98 \mathrm{~m} / \mathrm{s}$. They argued, that adding more deceleration stages would reduce the velocity even further.

This proof-of-principle experiment showed the feasibility of Stark deceleration and initiated todays application in many experiments as important decelerating step. Thereby, a large variety of molecules can be decelerated to velocities low enough to trap them. Among the decelerated molecules are for example $\mathrm{ND}_{3}[66]$, $\mathrm{OH}[67,68]$ and $\mathrm{SO}_{2}$ [69]. Not only the variety of species increased, but also the technology itself advanced. Stark decelerators have started as rather large devices with typical spacing of the stages in the range of $1 \mathrm{~cm}$ and an overall size around $1 \mathrm{~m}$. Today, they can be build in small scales when space-limitations demand it. Systematic miniaturization has lead to a Stark decelerator with only $11 \mathrm{~cm}$ length, which is approximately 10 times smaller than the conventional ones [70]. It still maintained a good efficiency with $90 \%$ of the kinetic energy removed. Even further size-reduction was achieved by implementing a Stark decelerator on a chip [71-73].

\subsubsection{Two-dimensional magneto-optical trap}

As the name already indicates, a magneto-optical trap combines a magnetic field with a light field. Considering a two-level system (see Fig. 1.6), the linear and inhomogeneous magnetic field $B$ leads to a Zeeman splitting of the magnetic $m_{F^{-}}$ states. The splitting is position dependent with a larger splitting further away from the trap center. The laser beams are red-detuned and the splitting brings them closer to resonance, enhancing the probability for absorption. This combination of laser light and magnetic fields results in a confining potential which also cools the atoms close to the Doppler limit.

The first magneto-optical traps where realized in 1987 by C. Cohen-Tannoudji, S. Chu an W. Phillips [4-6]. Ever since, magneto-optical traps are highly successful as a three-dimensional trap as well as in two dimensions creating high-flux atom beams. One big advantage over other methods like Zeeman slowers or Stark decelerators (see Chap. 1.4.1 and 1.4.2) is that the two-dimensional magneto-optical trap can produce beams which are radially cold. Combined with a pushing- and retarding beam (see Chap. 2.8), the velocity is widely adjustable. A two-dimensional 


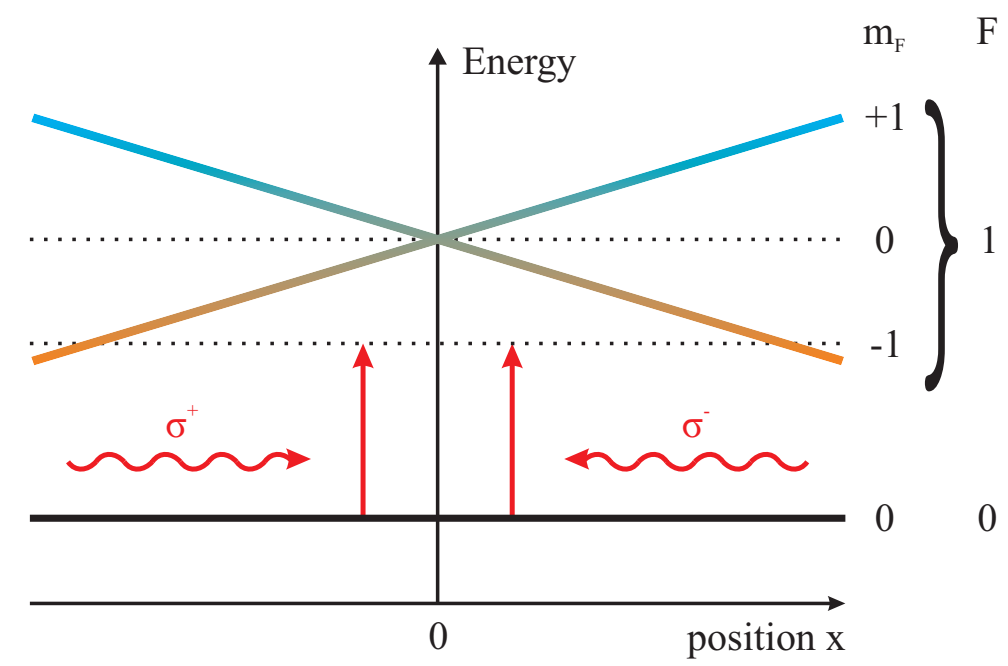

Figure 1.6.: Principle of a magneto-optical trap. A linear magnetic field results in an Zeeman shift of the atomic $m_{F}$ states in a two-level atom. This causes a favorable change in the probability to absorb a photon from the left or right depending on the position, resulting in an average force toward the trap center. The Doppler effect of the moving atoms leads to an increased probability to absorb a photon from a counter-propagating red-detuned light field. This leads to a friction force cooling the atoms.

magneto-optical trap can not only be used to create a continuous atomic beam, but the principal can also be applied to already existing atomic beams to further reduce their temperature. In this scheme, the guided atoms are adiabatically compressed in the radial direction which increases their temperature. Two pairs of perpendicular light beams reduce the temperature using the principles described above and the following adiabatic expansion results in a reduced temperature. An increase in phase-space density by more than a factor of 30 of a guided chromium beam was reported by A. Aghajani-Talesh and his colleagues using this method [74].

\subsubsection{Sympathetic cooling of molecular ions}

Cooling molecules is a promising but also challenging task. The rich internal structure hinders the application of laser cooling techniques, that are extremely successful when cooling atoms. Among the promising alternatives are photoassociation of cold atoms [75], buffer gas cooling (see Chap. 1.3.1) and Stark deceleration (see Chap. 1.4.2). With photoassociation, cold molecules can only be produced from laser-coolable atoms, eliminating many interesting candidates. Buffer gas cooling and Stark deceleration on the other hand can only produce relatively hot samples 
of molecules $(\sim 100 \mathrm{mK})$. A way to circumvent these limitations is to use molecular ions. These can easily be stored for minutes and continuous sympathetic cooling allows for low temperatures. Such an experiment was proposed in 2009 [76] and experimentally realized in 2013 [77] by E.R. Hudson and colleagues.

In this experiment, $\mathrm{BaCl}^{+}$molecules are cooled to translational temperatures in the millikelvin range with a ground state occupancy of about $90 \%$. This is achieved by loading a sample of $\mathrm{BaCl}^{+}$ions into a linear quadrupole ion trap. Then photodissociation is used to create $\mathrm{Ba}^{+}$ions that are co-trapped. Those ions are laser-cooled and in turn sympathetically cool the remaining $\mathrm{BaCl}^{+}$translationally. A co-located MOT can be turned on, trapping a cloud of neutral Ca atoms at the same location. The short-range collisions with the highly polarizable $\mathrm{Ca}$ atoms result in internal-state cooling. because of the combination of a magneto-optical trap (MOT) and an ion trap, they coined the term MOTion trap. This is a versatile tool to cool molecular ions expanding the range of molecular species coolable to the millikelvin range. However, even though this is a great progress, a variety of interesting molecules are still unaccessible. The laser light from the MOT can cause heating for many molecules with a rich internal structure. Thus, a dark and continuous reservoir is highly desirable.

\subsection{Continuous and dark cooling}

Here, the ${ }^{3} \mathrm{He}-{ }^{4} \mathrm{He}$ dilution refrigerator is described exemplarily as a continuous and dark cooling scheme.

\subsection{1. ${ }^{3} \mathrm{He}-{ }^{4} \mathrm{He}$ dilution refrigerator}

The dilution refrigerator makes use of the phase separation a ${ }^{3} \mathrm{He}-{ }^{4} \mathrm{He}$ mixture undergoes when cooled under roughly $870 \mathrm{mK}$. The ${ }^{3} \mathrm{He}$ reaches this temperature in a series of cooling baths. Two phases form, a concentrated phase, the ${ }^{3} \mathrm{He}$-rich phase and a diluted phase, the ${ }^{3} \mathrm{He}$-poor phase. The concentrated phase contains practically $100 \%{ }^{3} \mathrm{He}$, while the diluted phase only contains about $6.6 \%{ }^{3} \mathrm{He}$ and $93.4 \%{ }^{4} \mathrm{He}$. The ${ }^{3} \mathrm{He}$ atoms can be moved between these phases using osmotic pressure. The useful effect is that the specific heat of a ${ }^{3} \mathrm{He}$ atom is larger in the diluted phase than in the concentrated phase, and thus the temperature is reduced if atoms pass from the concentrated phase to the diluted phase.

Up to date dilution refrigerator can reach temperatures as low as $2 \mathrm{mK}$ [78]. It is the only continuous refrigeration method to reach temperatures below $300 \mathrm{mK}$ without the use of laser light [79]. 


\subsection{Summary}

Experiments that cool atoms and molecules continuously and entirely without the use of laser light become increasingly rare, when the temperature drops. In particular, a continuous cooling scheme in the microkelvin regime without the application of resonant light is still not available. The short introduction to a ${ }^{3} \mathrm{He}-$ ${ }^{4} \mathrm{He}$ dilution refrigerator in this sections describes the coldest, dark and continuous cooling scheme to date at a temperature of just below $2 \mathrm{mK}$. 


\section{Experimental apparatus}

The experiment described in this thesis runs on an apparatus with several subsystems. The different parts of the design are described in this chapter, starting with an overview of the experimental concept in Chap. 2.1. The vacuum system in which the experiment takes place is found in Chap. 2.2. The laser system supplying light for cooling, manipulation and detection is described in Chap. 2.3. A key component of the experiment is the mesoscopic atom chip and its current control is presented in Chap. 2.4, followed by the computer based control of the experimental sequence in Chap. 2.5. An overview over the atom chip traps in general in Chap. 2.6 introduces the mesoscopic atom chip of this experiment in Chap. 2.7. This atom chip generates almost all magnetic fields for trapping and manipulation of the atoms including part of the double magneto-optical trap setup described in Chap. 2.8. Before the atoms can be used for further experiments, final cooling and preparation has to be carried out, as described in Chap. 2.9.

\subsection{Experimental concept}

The fundamental goal of the apparatus is the investigation of novel concepts to trap and cool neutral atoms. To date, there are several ways to trap atoms, including light or electric fields. But one of the most widely used methods for trapping neutral atoms is the application of magnetic fields. It takes advantage of magnetic-field dependent shifts of the energy levels of the atom (see Chap. 2.6). The necessary magnetic fields for trapping and manipulation can be generated according to Biot-Savarts law by a current-carrying wire structure. These wire structures are roughly dividable into two main groups with their distinct advantages and disadvantages (see Fig. 2.1). The first group contains coil based setups where coils with multiple windings are placed in a variety of arrangements around the trapping region. These setups are usually comparably large structures, which produce smooth fields with a large trapping volume. On the downside, the switching times of large coils are not negligible due to their inductance. These setups also lack versatility, both in the sense that the field configurations achievable with the given coils are rather restricted, as well as that the fields have a large spatial extension and are thus not well confined. The other group comprises the chip-based traps. Here, microscopic, lithographically produced wire structures are placed close to the atoms. With these planar wire structures, external magnetic fields are not necessary so supply the magnetic fields. The production method and 
the small size allow for complex geometries and versatile trap setups. The chips can generate large magnetic field gradients due to the proximity to the atoms. They can achieve fast switching times due to the small inductance compared to the coil-based setups. Unfortunately, the small chip size dramatically limits the achievable trapping volumes and the one sided geometries lead to distorted fields.

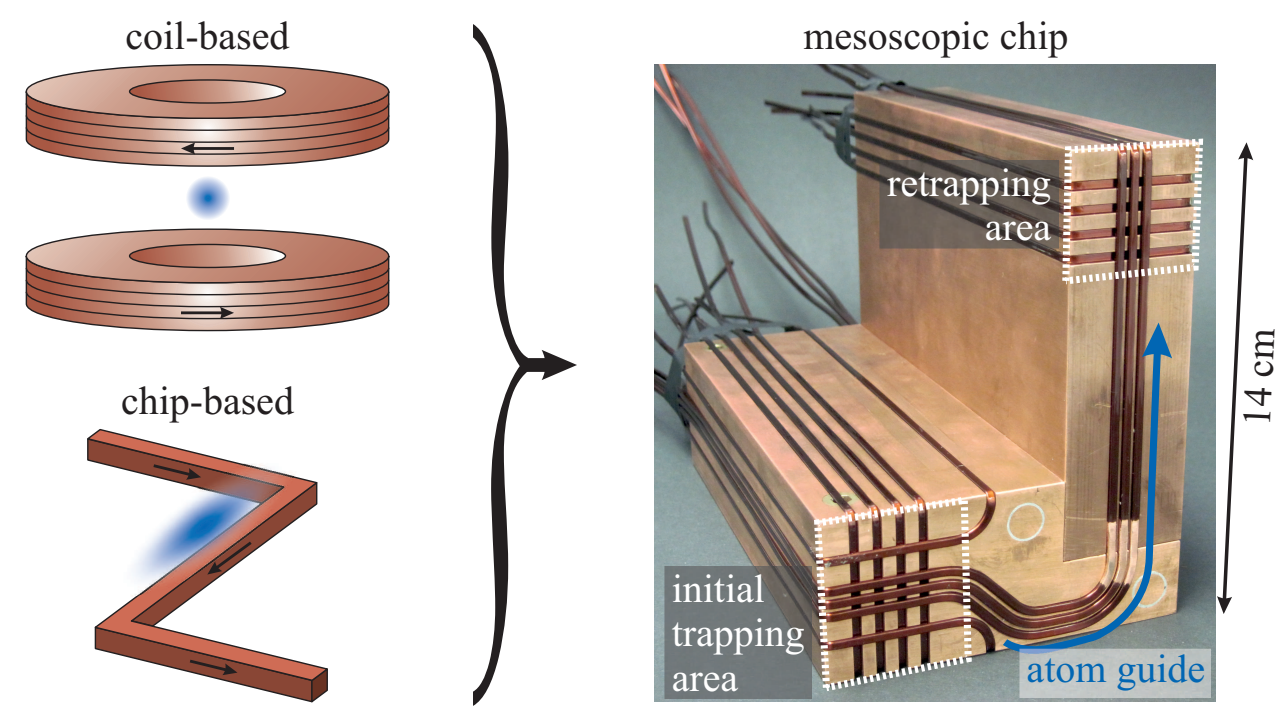

Figure 2.1.: Experimental concept. The advantages of coil-based and chipbased magnetic traps are combined in a mesoscopic atom chip, which allows for fast cycle times, versatile fields and large trapping volumes. The usage of the chip is divided in three main areas, the initial trapping area with further preparation, the atom guide and an area to retrap the atoms.

A combination of the benefits from the coil-based and the chip-based setups presents a new approach which pursued with this experimental apparatus. In this approach, we employ mesoscopic wire structures which utilize many of the chip-based principles to generate versatile fields with strong gradients. The large size on the order of a few ten centimeters still allows for large trapping volumes normally only achieved in coil-based setups. Furthermore, additional wires are used to compensate for the main trap distortions and produce smooth fields especially important for magneto-optical traps.

The mesoscopic atom chip used in the experiment is described in detail in Chapter 2.7 but is briefly introduced here (see Fig. 2.1) to motivate the different aspects of the setup. It consists of three main parts. First, an area to initially trap the atoms and prepare them for the following steps. This includes further cooling, state preparation and mode matching. Second, a guide to transport the atoms to a different region. This region features stray-light protection and improved vacuum 
conditions. Even though the guide connects the two regions, it needs to maintain the conditions in both. And third, a trapping region, where experiments towards continuous loading schemes are carried out (see Chap. 3).

To realize these different areas, a planar structure is built out of copper wires with a rectangular cross-section of $1.5 \mathrm{~mm} \times 2.5 \mathrm{~mm}$. The wires are mounted on an L-shaped, solid copper block, both for mechanical stability and cooling. The copper block has outer dimensions of $11 \mathrm{~cm} \times 14 \mathrm{~cm} \times 18 \mathrm{~cm}$ and is water cooled to support efficient heat dissipation. This mesoscopic atom chip can supply all the necessary magnetic fields for the experiments starting with the initial trapping.

\subsection{Vacuum system}

Atom optics experiments need to be performed under ultra-high vacuum to suppress collisions with the background gas, which will otherwise limit the lifetime of the atomic ensemble strongly. The vacuum chambers that are used in these experiments are often specially designed to fulfill individual requirements. Every chamber needs optical access for trapping, manipulation and detection. It may also need to accommodate for additional ways to trap atoms, like electric or magnetic traps. Vacuum pumps have to be integrated to reach and maintain a low-pressure environment.

Among the special requirements for the vacuum chamber of this experiment are large viewports to realize large trapping volumes in the magneto-optical trap, a stray-light protection for one part of the vacuum chamber and a possibility to mount the mesoscopic atom chip as close to the trapped atoms as possible. All these requirements were fulfilled in a custom design shown in Fig. 2.2.

The vacuum chamber consists of three separate areas for the trapping and manipulation of atoms: A glass cell for the 2D-MOT, a 3D-MOT trapping and launching area and an area for retrapping the atoms. A top view of the vacuum chamber is drawn in Fig. 2.2 and the whole mounted chamber is illustrated in Fig. A.1. The glass cell used for the 2D-MOT is an anti-reflection-coated quartz glass cell with good access for the 2D-MOT beams. Three independent rubidium dispensers are mounted inside, facing the trapping volume of the 2D-MOT. The 2D-MOT glass cell is connected to the main chamber via a differential pumping stage, which protects the main vacuum chamber from the comparably large pressure of about $10^{-7}$ mbar in the 2D-MOT which is optimal for its operation [80]. Additionally, the tip of the pumping stage has a $45^{\circ}$-angle and a polished surface to act as a mirror for the longitudinal 2D-MOT beams. The pumping stage separates the higher pressure in the 2D-MOT from the low-pressure of about $10^{-10} \mathrm{mbar}$ in the 3D-MOT area. The 3D-MOT area is part of the main chamber, separated from the retrapping area by a metal divider. This divider has a bent opening which blocks the direct line of sight but still enables the guiding of atoms through it. This opening acts as a differential pumping stage which enables a pressure drop 


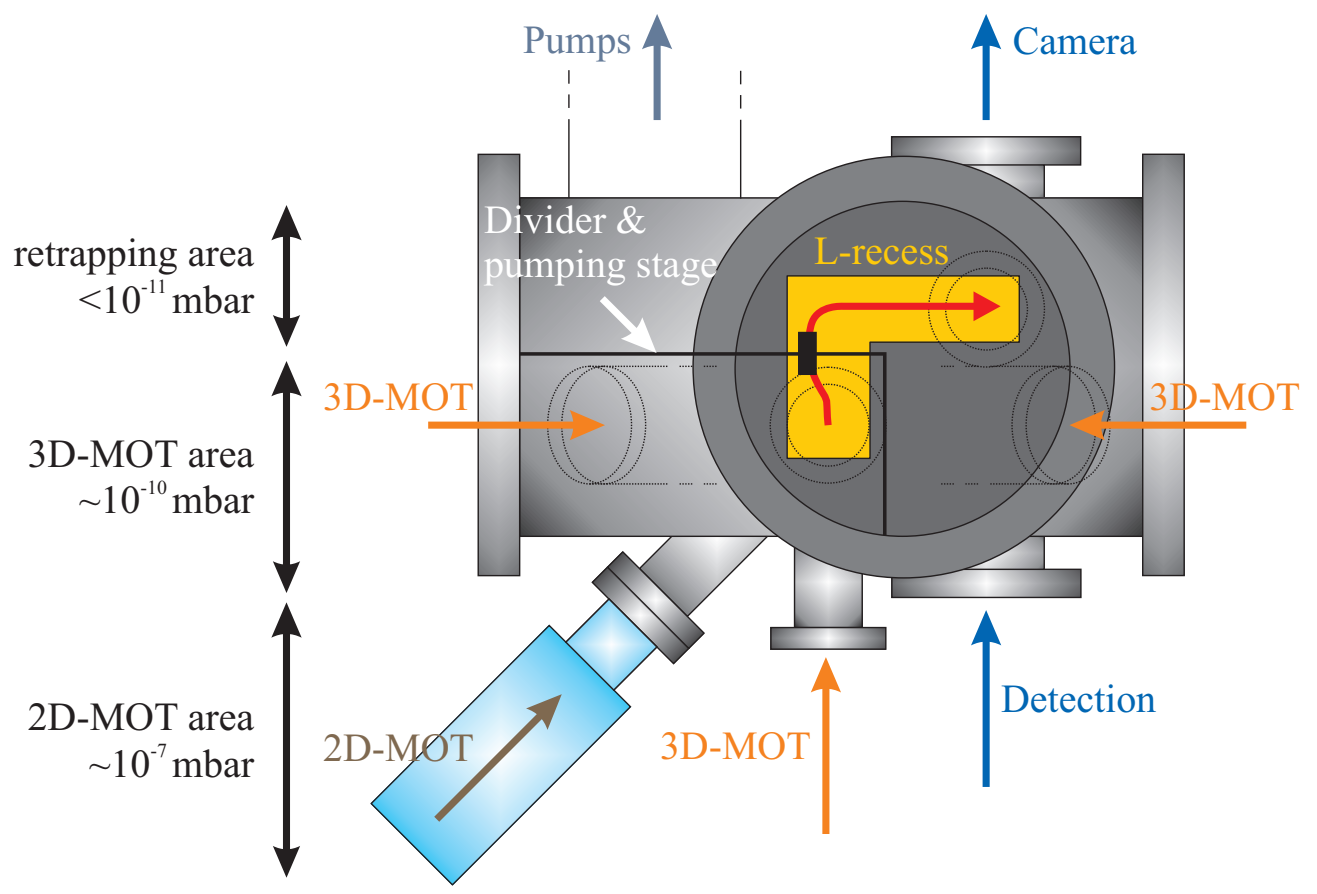

Figure 2.2.: Top view of the different vacuum chamber areas. The vacuum chamber is divided into a 2D-MOT area, a 3D-MOT and launching area and a retrapping area, each with different vacuum conditions. The atom guide (red) reaches from the 3D-MOT site to the retrapping region.

by more than a factor of ten between the 3D-MOT and the retrapping area. The entire divider plate has a rough $\mathrm{TiO}_{2}$-coating to maximize stray-light absorption. Mounted above the 3D-MOT area is a flange with an L-shaped recess reaching down to the trapping region and spanning over the opening in the divider to the retrapping area (see Fig. A.2). This recess is exactly shaped to accommodate the mesoscopic atom chip (see Chap. 2.7). Thus, the chip can be mounted close to the atoms, but still outside of the vacuum chamber, separated only by a $500 \mu \mathrm{m}$ steel foil. Thus, no vacuum feedthroughs are needed for the chip wires and their watercooling, simplifying the design and enabling better vacuum conditions. The retrapping area is spacious enough to support a long atom guide for different experimental configurations and five viewports at the end of the guide for further manipulation and detection.

The aforementioned pressures are produced by a combination of different pumps. To reach a low pressure initially, a turbomolecular pump was mounted behind an ultra-high vacuum valve. After this initial pumping, the system is closed and the pressure is maintained by two ion getter pumps. Additionally, a titanium sublimation pump in the retrapping region is available to reach and maintain the 
ultra-high vacuum.

\subsection{Rubidium laser system}

A laser system for cold atom optics experiments has to provide precise and reliable laser light with different frequencies. Each experimental step needs its own set of laser beams with different frequencies and powers. Among the experimental steps needing light fields are the operation of the 2D-MOT, the 3D-MOT, molasses cooling, state preparation and detection. All of these frequencies are chosen in the range of the ${ }^{87} \mathrm{Rb}$-D2-line from the ground state $\left|5^{2} S_{1 / 2}\right\rangle$ to the excited state $\left|5^{2} P_{3 / 2}\right\rangle$ with a wavelength of $780.241 \mathrm{~nm}$. The laser light has to be computer controllable and due to the dynamic nature of the experiment, special care has been taken to ensure fast and precise dynamical control.

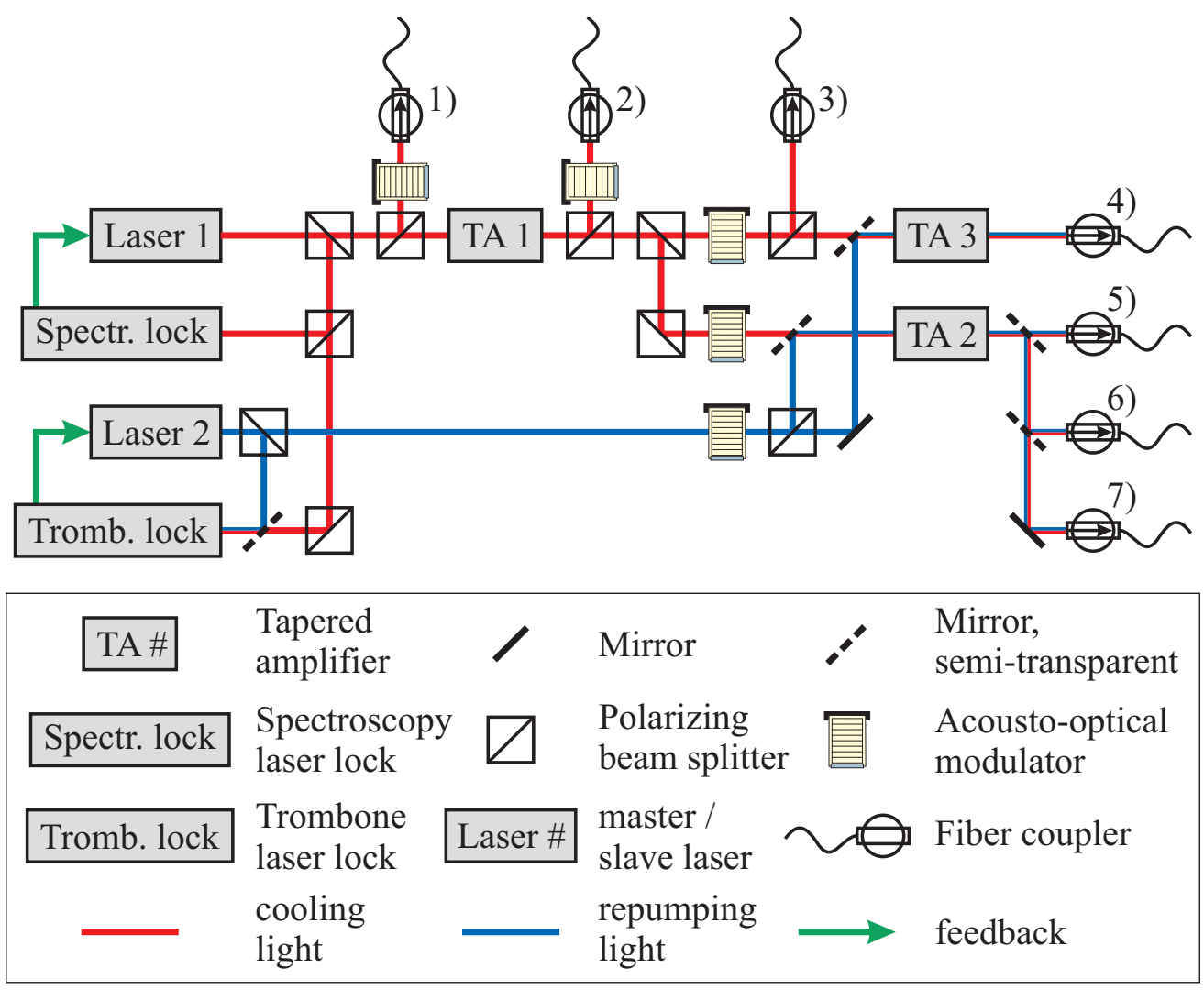

Figure 2.3.: Main components of the optical setup. This simplified picture shows the generation and distribution of the main laser beams to the experiment and the mixing of cooling and repumping light. The purpose, power and frequency of the light in the numbered fibers is given in Tab. 2.1. 


\begin{tabular}{|c|c|c|c|}
\hline & PuRPose & FREQUENCY & POWER \\
\hline 1 & $\begin{array}{l}\text { absorption detection in } \\
\text { retrapping area }\end{array}$ & $\begin{array}{l}\text { resonant on the transition } \\
\qquad F=2 \rightarrow F^{\prime}=3\end{array}$ & $0.25 \mathrm{~mW}$ \\
\hline 2 & optical pumping & $\begin{array}{l}\text { resonant on the transition } \\
\qquad F=2 \rightarrow F^{\prime}=2\end{array}$ & $0.7 \mathrm{~mW}$ \\
\hline 3 & $\begin{array}{l}\text { horizontal cooling light of the } \\
\text { 3D-MOT and optical molasses }\end{array}$ & $\begin{array}{c}6.2 \Gamma \text { to } 15 \Gamma \text { red-detuned to } \\
\text { the transition } \\
F=2 \rightarrow F^{\prime}=3\end{array}$ & $90.5 \mathrm{~mW}$ \\
\hline 4 & $\begin{array}{l}\text { cooling and repumping light } \\
\text { for the } 45^{\circ} 3 \mathrm{D}-\mathrm{MOT} \text { beams } \\
\text { from below, optical molasses } \\
\text { and fluorescence detection }\end{array}$ & $\begin{array}{l}\text { resonant to } 15 \Gamma \text { red-detuned } \\
\text { to the transition } \\
F=2 \rightarrow F^{\prime}=3 \text { and } \\
\text { resonant on the transition } \\
F=2 \rightarrow F^{\prime}=2\end{array}$ & $341 \mathrm{~mW}$ \\
\hline 5 & cooling and repumping light & $3.2 \Gamma$ red-detuned to the & $4.0 \mathrm{~mW}$ \\
\hline 6 & for the pusher and retarder (5), & transition $F=2 \rightarrow F^{\prime}=3$ & $196 \mathrm{~mW}$ \\
\hline 7 & $\begin{array}{l}\text { the horizontal (6) and vertical } \\
(7) \text { beam of the } 2 \mathrm{D}-\mathrm{MOT}\end{array}$ & $\begin{array}{l}\text { and resonant on the } \\
\text { transition } F=1 \rightarrow F^{\prime}=2\end{array}$ & $190 \mathrm{~mW}$ \\
\hline
\end{tabular}

Table 2.1.: Purpose, frequency and power of the light after the fibers to the vacuum chamber. The numbers correspond to the fibers in the simplified optical setup depicted in Fig. 2.3.

The laser system and its components have been described previously [81,82]. The setup is depicted in Fig. 2.3. The main initial source of laser light is an external cavity diode laser [83] locked to a Doppler-free saturation spectroscopy. It is locked $200 \mathrm{MHz}$ below the cross-over line from the $|F=2\rangle$-state to the $\left|F^{\prime}=1\right\rangle$-state and the $\left|F^{\prime}=3\right\rangle$-state (see Appendix Fig. B.1 for the level scheme with all relevant transitions). This laser light can be shifted by acousto-optic modulators (AOM) to all the desired frequencies driving transitions from the $|F=2\rangle$-state. This includes light for cooling, optical pumping and detection. The gap of $6.8 \mathrm{GHz}$ between the $|F=1\rangle$ and $|F=2\rangle$-state is too large for acousto-optical modulation and a second ECDL has to be used to drive all transitions starting from the $|F=1\rangle$-state. This laser is needed to pump atoms back into the cooling cycle and to detect atoms in the $|F=1\rangle$-state. It is stabilized with a microwave interferometer to the master laser with a fixed offset of $6.9 \mathrm{GHz}$ in a trombone lock configuration [84]. The replacement of the second ECDL laser by a microwave modulation of a tapered amplifier current is described in Chap. 4.

The low-power output of the two ECDLs is amplified with three tapered amplifiers (TA) with individual purposes. The first TA is used to preamplify the cooling light for the two other TAs and to provide slightly detuned cooling light without repumping light for the horizontal 3D-MOT beam. The second TA amplifies the light for the 2D-MOT, a combination of detuned cooling light and resonant 
repumping light. The third TA amplifies again the detuned cooling light for the 3D-MOT but with added repumping light. The detunings are realized individually for each TA by AOMs and are matched to the temperature of the atoms in each step. The light is finally sent via fibers to the vacuum chamber on a different table.

\subsection{Electronics and current control}

In chip based experiments, the current control for the atom chip wires plays an important role. Rapid switching is necessary for the dynamic trap setups. At the same time good current stability is essential to minimize noise and thus prolong the trap lifetime. In its current setup, the mesoscopic atom chip has nine independent wires which can be supplied with positive and negative currents up to $\pm 150 \mathrm{~A}$. Additionally, the chip features three special T-shaped wires allowing for currents of up to $150 \mathrm{~A}$, but only in one direction and with three connections each to support different current paths. An overview of the electronic concept is given in Fig. 2.4 while the different components are described below.

current to generate magnetic fields

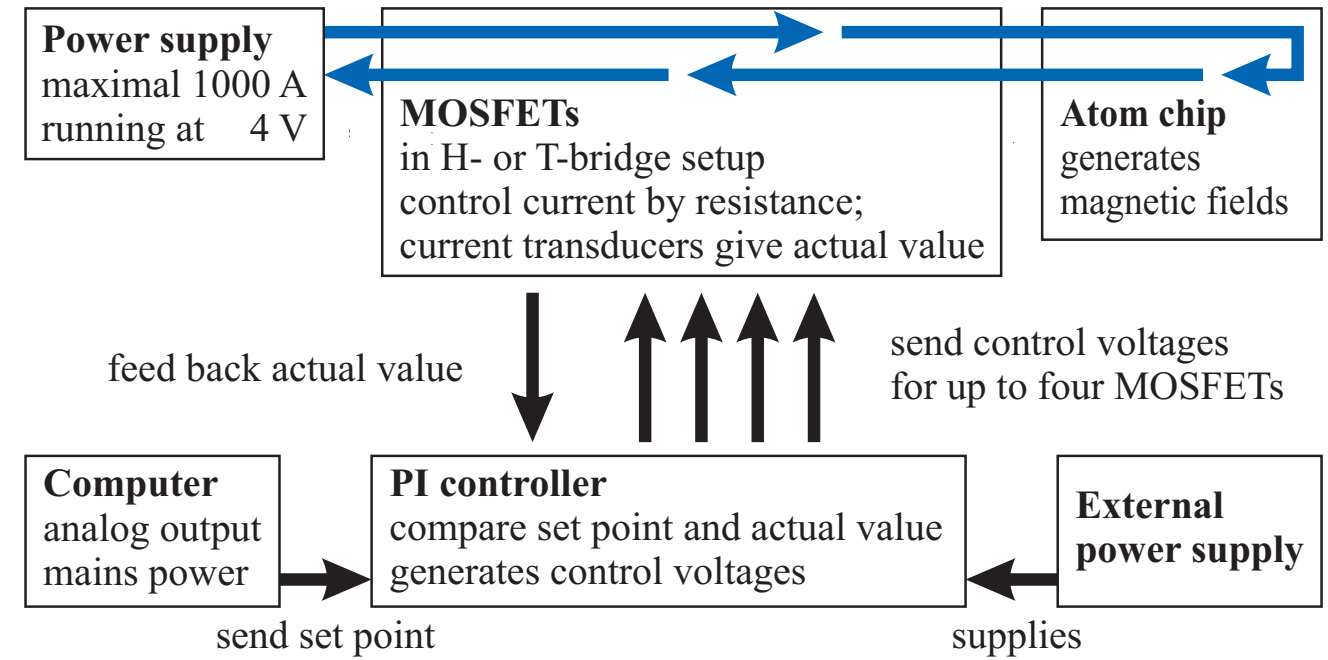

Figure 2.4.: Generation and control of the electric atom chip currents. The current of a high-current power supply is controlled by MOSFET circuits before it is send through the atom chip. Different MOSFET arrangements are used to support different wire geometries. An analog PI controller processes the feedback value from a current transducer and the set point from a computer control. 


\subsubsection{Current control with MOSFET circuits}

The requirements for the two different wire controls can be fulfilled with a set of metal-oxide-semiconductor field-effect transistors (MOSFET) in an appropriate logic. Having controllable currents in one wire in both directions can be achieved by four MOSFETs in an H-bridge configuration (see Fig. 2.5a). In this setup, four MOSFETs are placed in an H-shape with the atom chip wire forming the bar of the H. By opening MOSFETs 1 and 4 and closing the others, the current flows in the positive direction $(+\mathrm{I})$. Similarly, by opening MOSFETs 2 and 3 only, the current flows in negative direction (-I). MOSFETs 1 and 2 are not only switchable but can be opened partly to control the strength of the current. The actual value of the current is derived from a current transducer (LEM LA 100-P/SP13) measuring the current in the feed line to the chip wire. A PI controller (see Chap. 2.4.2) derives the control value for the four MOSFETs from this single signal. The current transducer can measure currents of up to $160 \mathrm{~A}$. By choosing high-power MOSFETs (IXYS IXFN200N07 and later IXFN200N10P), these currents of up to $160 \mathrm{~A}$ can be realized with this setup. The current is provided by a constantvoltage power supply (TDK Lambda GEN-7.5-1000-MD-3P400) with a maximal current of $1000 \mathrm{~A}$ and a maximal voltage of $7.5 \mathrm{~V}$. During normal operation, the power supply runs at a fixed voltage of $4 \mathrm{~V}$ to minimize heat dissipation.

a)

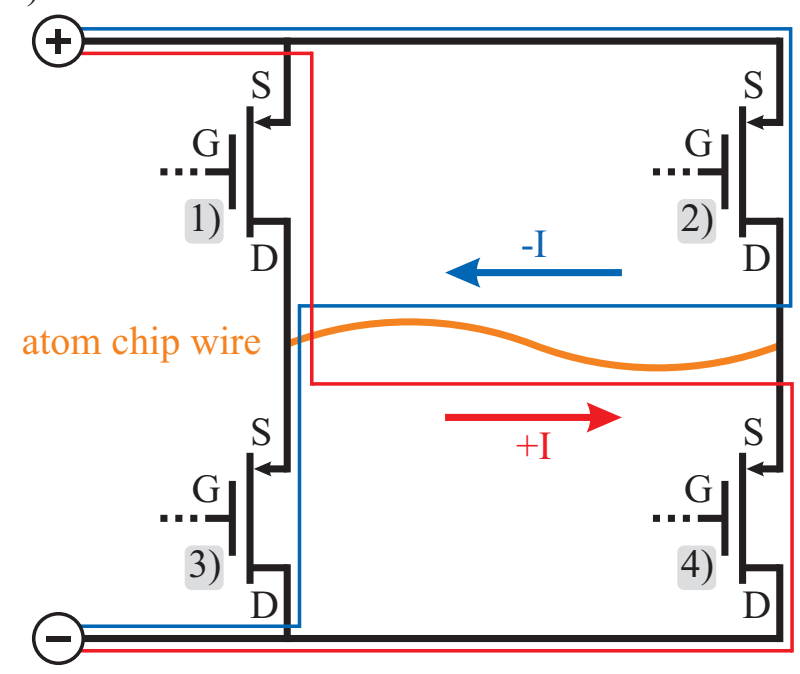

b)

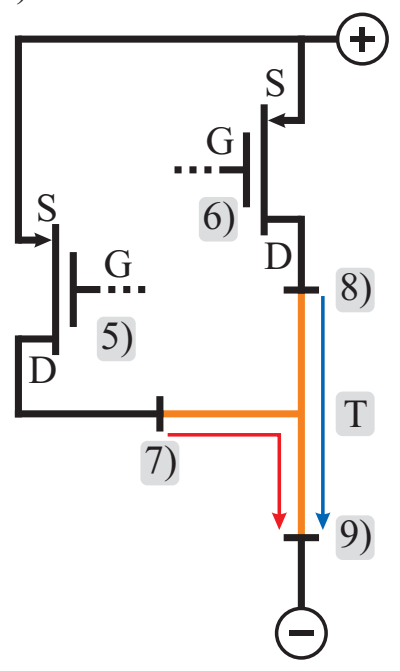

Figure 2.5.: Two MOSFET circuits for the atom chip current control. a) H-bridge. The pairwise switching of the MOSFETs allows for bidirectional control of the current. b) T-Shape circuit. This circuit supports one T-shaped guide wire with currents with a fixed polarity but with different currents in the separate "arms of the T".

The second configuration, that has to be operated is a T-shaped three-way wire 
where the currents are added in one arm of the $\mathrm{T}$. This connection is used for the three guide wires on the atom chip (see Chap. 2.7). Two currents, one from point 7 to 9 and the other from 8 to 9 are added in the arm from point $\mathrm{T}$ to 9 but are otherwise unaffected by each other (see Fig. 2.5b). This is achieved by having one MOSFET for each arm with an independent controller for each. This is different from the $\mathrm{H}$-bridge configuration with a single current transducer signal and PI controller for all four MOSFETs. Here, each feed line to the T-wire has its own current transducer and PI controller. The direction of the current is fixed in this configuration, but by switching of the supply polarity and the source (S) and drain (D) connections of the MOSFETS, T-wires with opposing currents can be realized. In the T-configuration, a single MOSFET failure can lead to chip damage. Such damage is prevented by melting fuses. The MOSFETs are mounted on a water-cooled copper plate to dissipate the heat due to the large currents.

\subsubsection{Analog PI controller for the chip currents}

The MOSFETs in the configurations described above need control voltages derived from the comparison between actual values from the current transducers and set points given by a computer control system. This comparison and control voltage generation is achieved by custom-built analog proportional-integral controllers (PI controller). The schematics and the basic working principle is depicted in Fig. 2.6. The PI controller generates four control voltages, one for each MOSFET in the H-bridge configuration. The output from operational amplifiers (OP) 9 and 11 have the same voltage, but inverted polarity and are analog values to control the strength of the current in MOSFETs 1 and 2 (see Fig. 2.5). The outputs from OPs 12 and 13 use the maximal output power of the OPs and are used to switch MOSFETs 3 and 4. In the T-shaped MOSFET configuration, both MOSFETs are controlled by individual PI controllers. Due to the unidirectionality, only the output from OP 9 is used here.

The low-noise PI controller reaches a control bandwidth for typical switching tasks of $20 \mathrm{kHz}$ to $30 \mathrm{kHz}$. The control bandwidth is reduced when the value of the current changes its sign. The linear response of the MOSFETs starts at control voltages above $4 \mathrm{~V}$ and to control the current, a larger voltages is needed. Thus, at each zero-crossing of the current the PI controller has to quickly gap a voltage of at least $8 \mathrm{~V}$, reducing its control bandwidth around $0 \mathrm{~A}$. This could be prevented in future PI controllers by not allowing the control voltage to be negative and by adding a constant voltage of $4 \mathrm{~V}$ to the output. For switching currents rapidly from $0 \mathrm{~A}$, a similar situation arises. This is preventable by applying a negligible current beforehand to prime the controller. In operation with the mesoscopic atom chip, the main speed limitation arises due to eddy currents in the copper piece holding the chip wires. A new mesoscopic atom chip is designed and set up to reduce this and other limitations and to add new features, as described in Chap. 5. 


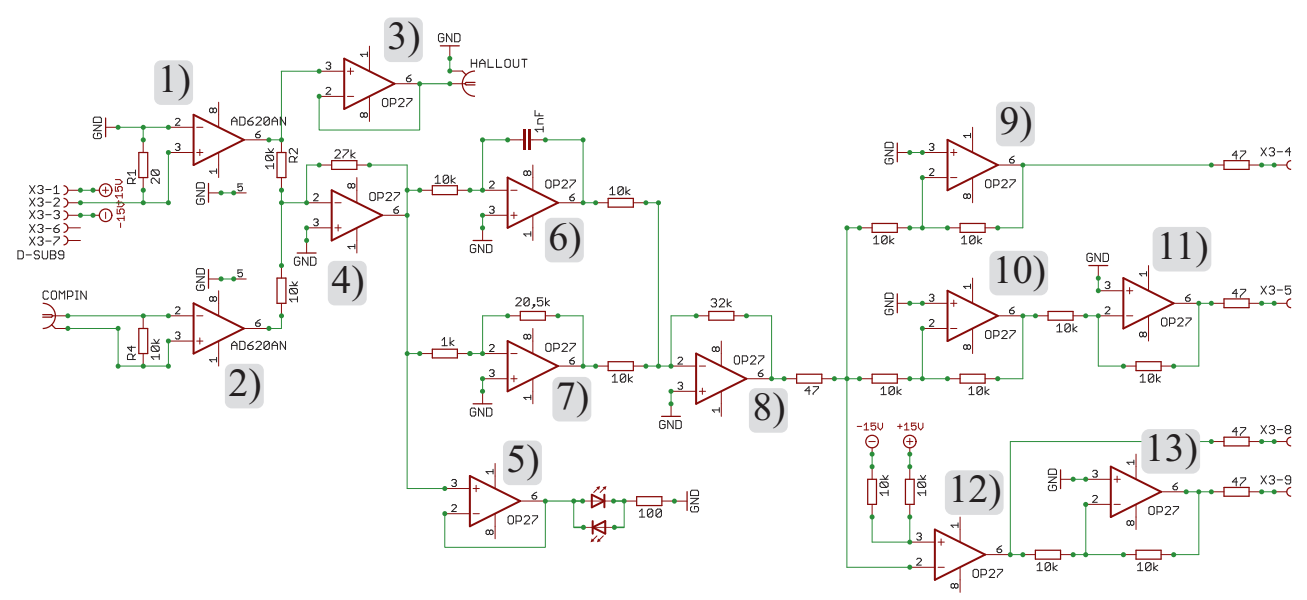

Figure 2.6.: PI controller for the atom chip currents. The set point from a computer control and the actual value from a current transducer are fed into the circuit via intrumentation amplifiers 1 and 2 and are then subtracted at OP 4 . OP 3 delivers a proportional monitoring output for the current from the current transducer. OP 5 operates two light-emitting diodes at the outside of the housing with the difference signal as error indicator. The response from the proportional (7) and integral part (6) are added using OP 8. OP 9, 10 and 11 generate an inverted and non-inverted output signal proportional to the error signal, while OP 12 and 13 generate strongly amplified opposing signals for switching.

\subsection{Computer-based experimental control}

Many experimental parameters need to be controlled, allowing for the operation of shutters, cameras and currents. Especially dynamically changing values need a fast and reproducible control, that only a modern computer system can handle. The mesoscopic atom chip (see Chap. 2.7) features numerous wires with independent, varying currents, so that not only digital signals for switching, but also analog control signals are necessary. A computer system capable of delivering these control signals is used to operate the experiment.

\subsubsection{Experimental control hardware}

The core hardware component is a field-programmable gate array (FPGA, model: Virtex-II 3M) on a commercial PCI-card (National Instruments NI PCI-7813R R Series Digital RIO) in a customary computer. Among the benefits of FPGAs are outputs with a high update rate and small jitter. A precise internal clock serves as a reference for all outputs and ensures reproducible signals. The employed 
FPGA has an internal clock with a cycle time of $40 \mathrm{MHz}$ with an accuracy of $\pm 100 \mathrm{ppm}$, an inferred minimal time step of $5 \mathrm{~ns}$ and a timing jitter of $250 \mathrm{ps}$. The FGPA card delivers a total of 160 binary output ports (TTL-standard) at four connectors. One of these is connected to a distribution module and thereby made available to the experiment as digital signals. The other three are converted into analog signals in sets of 40 digital channels to 16 analog channels each. This is accomplished with digital-analog converters (National Instruments NI 9263) which sit in groups of four inside a distribution housing (National Instruments NI 9151 $\mathrm{R}$ Series Expansion Chassis). The analog outputs generate voltages of $\pm 10 \mathrm{~V}$ with a resolution of 16 bit and a refresh rate of $100 \mathrm{kSamples} / \mathrm{s}$. For further connectivity, the computer has an additional plug-in card with a General Purpose Interface Bus (GPIB / IEEE-488), that is able to deliver ASCII-commands and is synchronized to the FPGA-output through the control software.

\subsubsection{Experimental control software}

The software running on the computer and the FPGA is programmed in the LabVIEW environment by National Instruments. The computer software displays a front end, from which all of the features are accessible. A matrix is used to control the experimental sequence, where the columns correspond to the time steps and the rows correspond to the different output channels. The digital channels can be set to LO or HI in each time step and stay constant during this time. The analog values can be varied within the time steps with a second order polynomial. They can also be calibrated to not only display the output voltage but also the desired target control parameter for ease of operation. The channels may be adjusted individually to match the delay of any controlled device. The GPIB commands are input as text in ASCII-fields and also have one line per output channel. The length of the time steps, all analog values as well as values in GPIB-commands can be varied stepwise throughout a sequence of automated experimental cycles. All relevant inputs from the front end can be saved to the hard disk and restored, automatically adjusting to the newest version of the program. Some modes of operation allow for an automatic save option. The cameras for detection are also triggered by this program, but the collection and analysis of the pictures is performed by a separate computer. A further description of the program can be found elsewhere with an emphasis on the FPGA and the digital channels [85] and the analog part [86].

\subsubsection{Automated experimental optimization}

One extensive feature of the computer control is the automatic optimization of the experimental operation through an algorithm. This algorithm solves an otherwise difficult task, the optimization of a desired experimental result that depends on 
many correlated parameters. Optimization by hand or by scanning the entire parameter space is a time consuming task, often too inefficient to yield satisfactory results. A more effective method to optimize the experiment is the use of the computer control system in combination with appropriate optimization algorithm. The algorithm that we developed for this task is called Limited Individual Lifetime Differential Evolution (LILDE) [87] and is based on the well established Differential Evolution algorithm (DE) $[88,89]$.

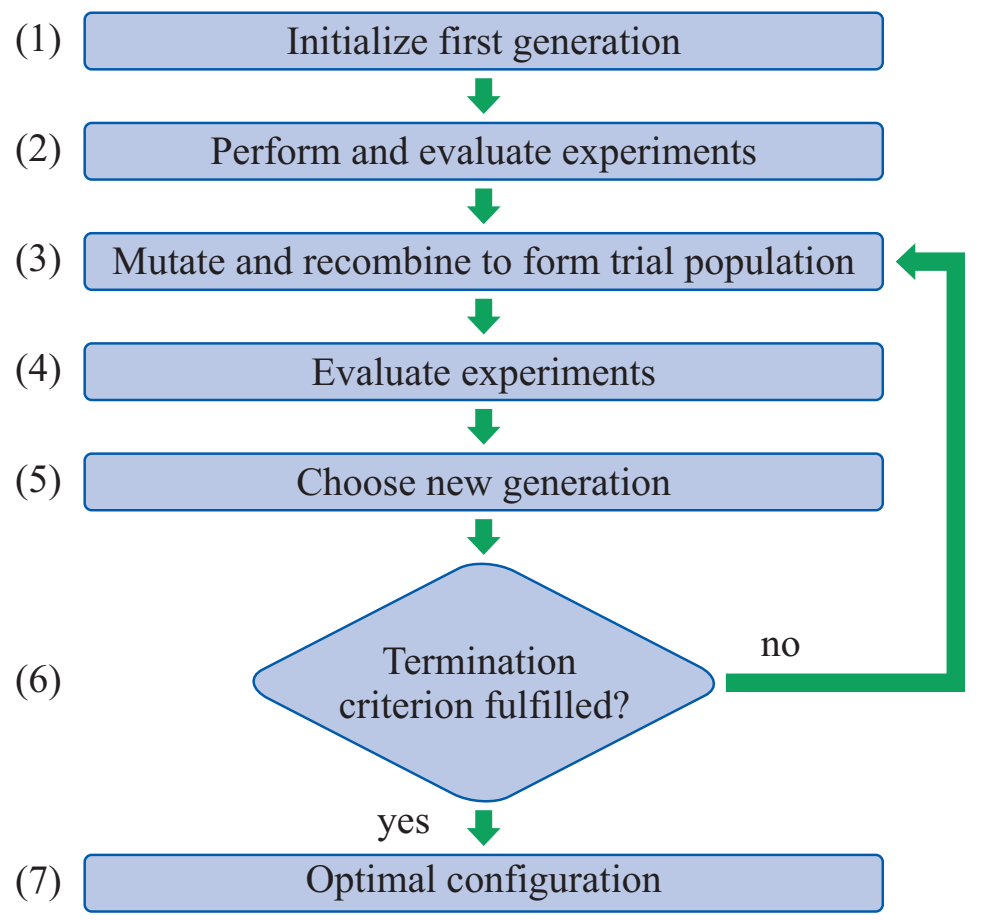

Figure 2.7.: Basic process of the Differential Evolution algorithm. (1) The first generation is initialized by a rondom distribution over the parameter space. (2) The performance of each candidate is evaluated. (3) A new trial population is formed through a mutation of the former. (4) The new trial population is also evaluated in the experiment. (5) The new candidate replaces its predecessor if it performs better. (6) Steps three to five are repeated until a termination criterion is fulfilled. (7) The optimal configuration is found.

The Differential Evolution algorithm is used for the global optimization of multidimensional, continuous functions with a heuristic approach. The basic working principle is shown in Fig. 2.7. At start, a first generation of vectors, each containing a complete set of experimental parameters, is initialized by randomly distributing the values over the allowed parameter space. Then, the vectors are evaluated through the sequential measurement of the vectors. Afterwards, a trial generation 
is created through a linear combination of the best vectors of the population that was just evaluated. This trial generation is also evaluated in the experiment. The performance is measured by a pairwise comparison with the old trial vectors and the better performing ones are chosen. The mutation, evaluation and performance selection is repeated until a termination criterion is fulfilled.

Among the benefits of the Differential Evolution algorithm are its ease of operation and robustness as well as its reliability to find an optimum. However, to use it in an experimental environment and not only with invariant functions, extensions had to be made in the LILDE algorithm to account for drifts and experimental noise. The main change is to limit the lifetime of each individual vector. This enables the algorithm to constantly adjust to drifting experimental conditions. In addition, the limited lifetime avoids the accumulation of vectors which accidentally perform disproportionately well due to technical noise.

The LILDE algorithm is implemented into the experimental control software and has been used to optimize many different experimental steps. It is especially beneficial in this experimental environment due to the short cycle times and the large number of correlated parameters, most notably the currents of the mesoscopic atom chip. It reliably finds good experimental solutions within hours, which was demonstrated for sets with up to 21 parameters. For example, the optimization was applied for the operation of a magneto-optical trap and the launch of atoms into the atomic guide (see Chap. 3.1).

\subsection{Atom chip traps}

\subsubsection{Development of atom chip traps}

Ideas of magnetic traps for neutral particles were formulated in the 1960s for neutrons and were realized in 1978 for the first time [90]. In the 1980s, magnetic trapping of neutral atoms became possible due to advances in laser cooling and the development of magneto-optical traps [91]. In 1995, the first Bose-Einstein condensates were created in magnetic traps [7-9]. These early magnetic traps were usually created with current-carrying coils, which are rather large and heat up considerably. The coils were usually placed outside of the vacuum chamber with a distance of at least several centimeters to the trap minimum. Even though atoms can be trapped in this way, a large distance can be disadvantageous. Consider a wire carrying a current $I$ generating a magnetic field at a distance $r$. Here, the magnetic field scales with $I / r$, and even more significant, the magnetic field gradient $\partial B / \partial r$ scales with $I / r^{2}$. Especially the magnetic field gradient is important, as it directly influences the trap frequencies and consequently the achievable densities. Thus, it is desirable to maximize the magnetic field gradient by a reduction of the distance $r$, which at the same time increases the energy efficiency. First suggestions where made in 1995 in the form of planar wire structures close 
to the trap center [92]. The development of atom chips followed and the distance shrunk to the range of micrometers. These microscopic atom chips are produced in a lithographic process, which is also used in the production of integrated circuits. This relation to "computer chips" helped to coin the term "atom chip" in 2000 [93].

Among the benefits of chip traps is a much stronger confinement than in macroscopic coils with the same current. This is especially beneficial for experiments with limited power supply, such as portable devices or experiments in zero gravity, which are solely battery-powered. A strong confinement is also essential for fast evaporational cooling and high cycle times. Atom chips close to the trap center can also deliver large gradients and fields that diminish quickly over short distances. This allows for complex geometries with several trap sites close to each other.

Even though quite successful, the miniaturization has some downsides. The proximity to the chip surface reduces the lifetime of the atoms. The distance from the trap center to the chip surface and the smaller fields also limit the trap size. Therefore, we decided to implement a chip with mesoscopic length scales. The wires have a cross section of $1.5 \mathrm{~mm} \times 2.5 \mathrm{~mm}$ and form structures in the centimeter range. This size combines the benefits of the coil traps and the chip traps: Large trapping volumes and a large number of atoms while maintaining the flexible trap configurations and large magnetic field gradients.

\subsubsection{Magnetic trapping}

Atoms feature a magnetic moment $\vec{\mu}$, which is especially strong in atoms with unpaired electrons, such as alkali metals. When exposed to an external magnetic field $\vec{B}$, the interaction with the magnetic moment results in an energy shift of the atomic levels [94]

$$
\Delta E=-\vec{\mu} \vec{B}
$$

According to quantum mechanics, the magnetic moment of the atom is quantized. This is taken into account by introducing the quantum number $m_{F}$ that represents the quantized projection of the total angular momentum $F$ onto the magnetic field axis. The quantum number $F$ defines the hyperfine states, while states with differing $m_{F}$ are called Zeeman states. The energy shift of the Zeeman states in a weak external magnetic field is

$$
\Delta E=g_{F} \mu_{B} m_{F}|\vec{B}|,
$$

with the Landé g-factor $g_{F}$ of the hyperfine state $F$ and the Bohr magneton $\mu_{B}$.

The Zeeman states can be divided into three groups according to the direction of the energy shift of the atomic level. This division is especially relevant for the magnetic trapping of atoms. When the atoms experience a magnetic field, those with magnetic moments aligned with the field $\left(g_{F} m_{F}<0\right)$ have lower energies 
in regions with higher fields. These atoms are accelerated in the direction of a magnetic field gradient and are thus called high-field-seeking states. The second group with $m_{F}=0$ is in first order not sensitive to magnetic fields. The third group with $\left(g_{F} m_{F}>0\right)$ is aligned, such that the atoms are accelerated in the opposite direction of the magnetic gradient. These are the so-called low-field-seeking states. Maxwell's equations do not allow for a local magnetic field maximum and therefore only atoms that are low-field-seeking are trappable in a static magnetic trap [38]. Therefore, preparation of atoms in one ore more trappable states is key for the magnetic trapping of the atoms (see Chap. 2.9).

\subsubsection{Principles of atom chip traps}

The following section describes the basics of atom chip traps and how they are able to produce magnetic trapping fields with current-carrying wires. The creation of a trap minimum using one wire with an external field as well as three wires without external fields is described. Also, the generation of a perpendicular field with four wires to complete a three-dimensional trap is given. For simplicity, all wires in this chapter are assumed to have vanishing thickness and infinite length.

\section{One wire with external field}
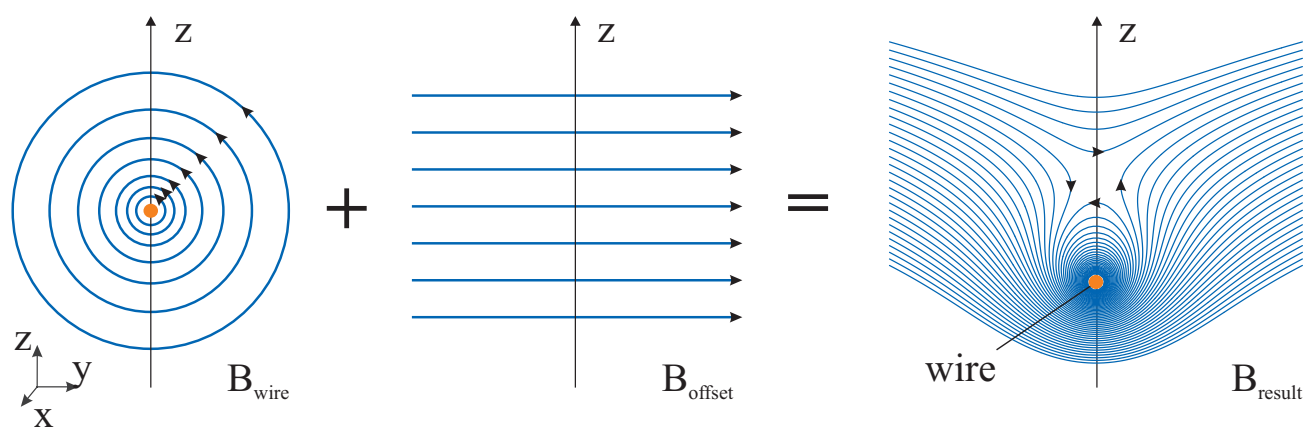

Figure 2.8.: Trapping principle with a single wire. The circular magnetic field generated by a current-carrying wire is superimposed with a homogeneous offset field. Both fields cancel above the wire and result in a quadrupole-like field around this minimum. The image is based on [95].

The simplest wire trap consists of a single wire with an external homogeneous field (see Fig. 2.8). A current-carrying wire along the x-axis produces a magnetic field with a magnitude [96]

$$
B_{\text {wire }}(r)=\frac{\mu_{0} I}{2 \pi r}
$$


where $\mu_{0}$ is the vacuum permeability, $I$ the current in the wire and $r$ the radial distance from the wire center. This field is superimposed with a homogeneous bias field $B_{\text {bias }}=B_{0}$ in y-direction. As the field $B_{\text {wire }}(r)$ weakens with the distance from the wire, there is a point where both fields have equal strength and thus cancel above the wire at the position

$$
z_{0}=\frac{\mu_{0} I}{2 \pi B_{0}} .
$$

Close to the minimum at $z_{0}$, the field is well approximated by a two dimensional quadrupole field with a gradient

$$
B_{\text {wire }}^{\prime}\left(z_{0}\right)=\left.\frac{\partial B_{\text {wire }}}{\partial r}\right|_{r=z_{0}}=\frac{2 \pi B_{0}^{2}}{\mu_{0} I} .
$$

The minimum of this quadrupole field stretches above the wire on a straight line at $\left(x, 0, z_{0}\right)$, where the magnetic field vanishes. Along this line, atoms in low-fieldseeking states can be trapped and guided. This trap presents the basic principle for all other wire traps.

\section{Chip trap without external fields}

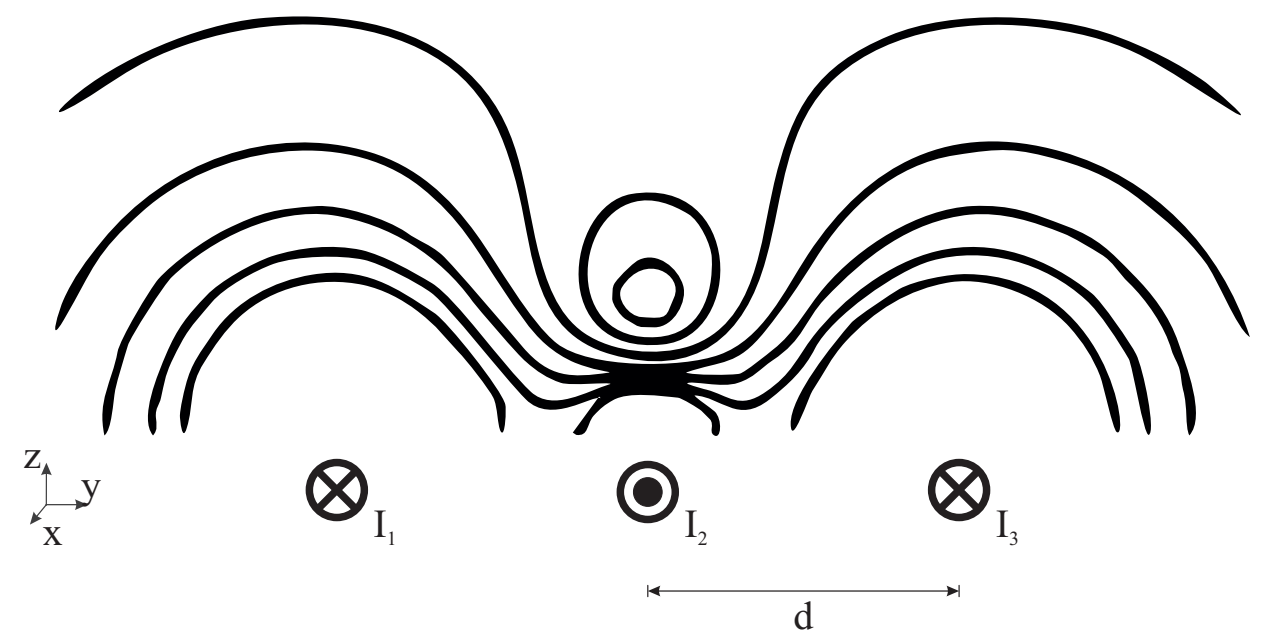

Figure 2.9.: Wire trap without external bias field. Three wires are used to generate a magnetic trap. The field from the outer wires replaces the external offset field. No further fields are necessary to form a trap [97].

It is often beneficial to avoid an external bias field as described in the trap above, but to generate the bias field also with a planar wire structure. In this way, the entire magnetic field is generated by the atom chip, increasing its flexibility and enabling several trap sites close to each other, each with an individual bias field. To 
do so, one can eliminate the external field and introduce two additional wires next to the central guide wire with currents in opposite direction (see Fig. 2.9). The fields of the two additional wires add up to a magnetic field with a homogeneous component above the central wire.

With the origin in the central wire, the position of the magnetic field minimum is at $[98]$

$$
y=0, \quad z=\frac{d}{2} \sqrt{\frac{1}{2 \beta-1}},
$$

where $d$ is the distance of the outer wires and $\beta=I / I_{\text {inner }}$ is the ratio between the current in the inner and outer wires. The radial magnetic gradient is given by

$$
B^{\prime}=B_{0}^{\prime} \frac{z^{2}}{d^{2}} \frac{4(2 \beta-1)^{2}}{\beta}=B_{0}^{\prime} \frac{2}{1+(2 z / d)^{2}} \quad \text { with } B_{0}^{\prime}=\frac{\mu_{0}}{2 \pi} \frac{I_{\text {inner }}}{z^{2}}
$$

Assuming the same $z_{0}$ as in Eq. (2.4), $B_{0}^{\prime}\left(z_{0}\right)$ becomes equal to the single wire case. This trap gains significantly in flexibility compared to the single wire setup. The trap center is movable in the z-direction by tuning the ratio of the currents in the outer and inner wire. It can be shifted in the y-direction by unbalancing the currents in the two outer wires. Assuming an equidistant spacing $d$ between the wires and independent currents in the wires from left to right $\left(I_{i}=I\left(\right.\right.$ wire $\left.\left._{i}\right)\right)$, the general position of a three-wire trap is [99]

$$
y=\frac{d}{2} \frac{I_{1}-I_{3}}{I_{1}+I_{2}+I_{3}}, \quad z=\frac{d}{2} \frac{\left[4 I_{1} I_{3}-\left(I_{1}+2 I_{2}+I_{3}\right)^{2}\right]^{1 / 2}}{I_{1}+I_{2}+I_{3}} .
$$

The three-wire trap is used to guide atoms and as a two-dimensional enclosure for a purely magnetic three-dimensional trap. To use it as part of a magnetooptical trap adjustments are necessary. Even though a quadrupole field is a good approximation for the three-wire trap close to the trap center, distortions become relevant quickly. This is especially critical if the magnetic field is combined with laser light to operate a magneto-optical trap (see Chap. 1.4.3). In that case, the magnetic field lines need to run parallel to the laser beams. Deviations of the angle limit the achievable number of atoms [100]. For large trapping volumes, it is thus beneficial to compensate the dominant distortion, the hexapole component of the field. The compensation can be done with a planar wire structure and was covered previously [101]. Two additional wires perpendicular to the three-wire trap are used to compensate the hexapole component and enlarge the trapping volume significantly $[82,102]$.

\section{Longitudinal quadrupole trap with four wires}

The three-wire trap described above can be used as a guide or as a two-dimensional enclosure for a three-dimensional trap. To close such a trap in the third direc- 


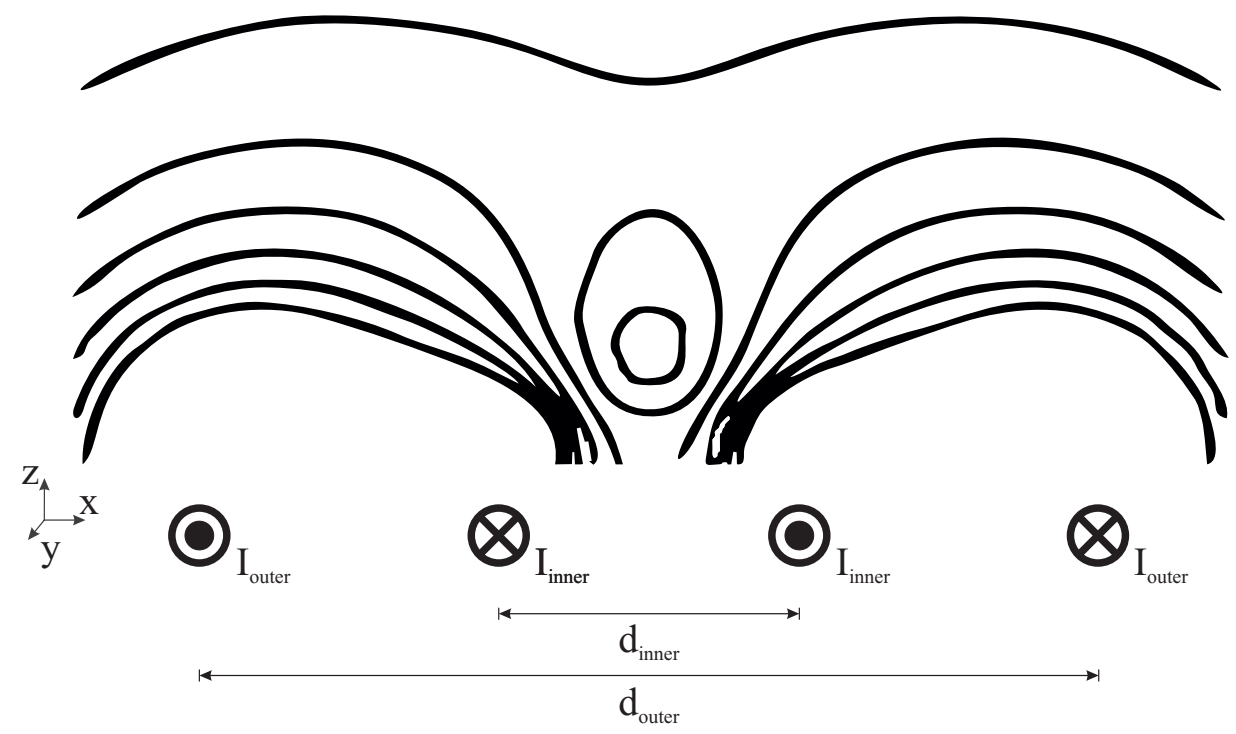

Figure 2.10.: Four wires generating a magnetic quadrupole. Four wires carrying currents in alternating directions generate a two-dimensional quadrupole, as required for a guide or for a three-dimensional trap [97].

tion requires an additional trapping field. This can for example be achieved by a quadrupole field generated by four perpendicular wires. The wires with alternating current directions produce a field perpendicular to the three-wire field, thus described in the x-z-plane (see Fig.2.10). With the origin between the central wires and a spacing between the inner wires of $d_{\text {inner }}=d$, the trap center position is at $[103]$

$$
x=0, \quad z=\frac{d}{2} \sqrt{\frac{\alpha-\beta}{\beta-1 / \alpha}} .
$$

Here, the ratio of the distance between the outer wires $d_{\text {outer }}$ to the distance of the inner wires is given by $\alpha=d_{\text {outer }} / d$. The ratio of the current in the inner and outer wires is given by $\beta=I_{\text {outer }} / I_{\text {inner }}$. In this case, the axial magnetic field gradient is [98]

$$
B^{\prime}=\frac{\mu_{0}}{2 \pi} \frac{I_{\text {inner }}}{d^{2}} \frac{16}{\beta\left(\alpha^{2}-1\right)^{2}} \sqrt{(\alpha \beta-1)^{5}\left(1-\frac{\beta}{\alpha}\right)} .
$$

A large gradient is desired for a tight confinement. The gradient is maximized for the optimal current ratio

$$
\beta_{\max }=u\left(1+\sqrt{1+u^{2} / 2}\right), \quad \text { with } u=\left(3 \alpha^{2}-1\right) / 8 \alpha
$$


In the case of equally spaced wires $(\alpha=3)$, the optimal current ratio is $\beta_{\max }=$ 2.45. Under these conditions, the gradient is maximized to

$$
B_{\text {max }}^{\prime}=4.16 \frac{\mu_{0}}{2 \pi} \frac{I_{\text {inner }}}{d^{2}} .
$$

This trap can be used as an atom guide with a tighter confinement than in the three-wire trap. This trap can also be used to complete a three-dimensional trap. The equidistant wire spacing allows for the implementation of a transport mechanism. By adding further perpendicular wires, an equal trapping field can be generated with every four adjacent wires. By adiabatically transforming the currents from one trap site to the next, a smooth transition is possible. This enables a magnetic "conveyor-belt" [104] for atoms.

\subsection{Mesoscopic atom chip}

The central element of the experimental apparatus is the mesoscopic atom chip. It is used to generate all magnetic fields besides the fields for the two-dimensional magneto-optical trap (2D-MOT). It produces the magnetic fields for the threedimensional magneto-optical trap (3D-MOT), for further magnetic confinement and guiding, as well as complex trapping geometries in the retrapping area. No external fields are needed for any of these steps. The different uses put different constraints on the design of the atom chip. The sometimes contradicting demands and their solution are presented in this chapter for the three main areas of the atom chip: The initial trapping and state preparation in the 3D-MOT area, the atomic guide and the retrapping area at the end of the guide (see Fig. 2.1). A further description of the chip design is given in references [82,102].

The first section of the atom chip is the initial trapping region. Here, a 3D-MOT is loaded, state preparation is performed and finally, the atoms are either loaded into a magnetic trap or phase-matched and launched into an atomic guide. Especially the operation of the 3D-MOT puts high demands on the size and purity of the magnetic quadrupole field. As magneto-optical traps become density-limited quickly [105], the only way to trap large amounts of atoms is to increase the trapping volume. Therefore, spatially large fields are needed, which have a sufficient distance to the next surface. Additionally, the magnetic field has to be a quadrupole field with only minimal distortions due to higher-order multipoles to ensure that the main magnetic axes are oriented in parallel to the laser beams. This is achieved with a three-wire trap (see Chap. 2.6.3) creating a two-dimensional quadrupole and an additional pair of parallel wires to compensate the hexapole distortions [102]. The trap is completed in the longitudinal direction with a four wire trap. The wire spacing was optimized by a multipole optimization, putting the magnetic trap center at $8 \mathrm{~mm}$ distance to the atom chip and creating a spherical trap with $2 \mathrm{~mm}$ radius. The magnetic field lines are in parallel to the laser 
beams for at least $4 \mathrm{~mm}$. This configuration can produce gradients of 11, 15 and $5 \mathrm{G} / \mathrm{cm}$ along the laser beams.

The magnetic trap and the guide have different demands on the magnetic fields. They do not need to be as pure as a for magneto-optical trap (MOT), because they do not have to be matched with light fields. They can also have a considerably smaller effective trapping volume, because the atoms are significantly colder and the density-limitations of MOTs do not apply. However, large gradients and a strong confinement are needed for good control over the atoms. These differing demands are met by pulling the trap center closer to the chip surface. As shown in Chapter 2.7, the strength of the field and the gradient depends strongly on the distance to the wires.

The outer compensation wires are thus not needed in later trapping stages and in the launch process has further advantages when loading the atomic guide. The guide is generated by the three inner wires used in the 3D-MOT, which also extend on the chip surface to the retrapping area. By using these wires only to provide the radial confinement in the magnetic trap and during the launch process, a mode match to the guide can be realized. After accelerating the atoms into the atomic guide utilizing the four perpendicular wires, they travel along the path through a bent narrow in a vacuum divider to the retrapping area. The divider separates the initial trapping and the retrapping area to ensure better vacuum conditions in the retrapping area and to protect it from stray light from the 3D-MOT.

In the retrapping area, continuous loading of a non-dissipative trap is investigated. To achieve this, another set of four perpendicular wires is placed at the end of the guide. These wires have the same spacing as the perpendicular wires in the initial trapping region. Several trapping schemes are tested, including schemes with a non-vanishing offset field in the trap center to reach low temperatures while minimizing Majorana spin-flip losses [106]. The launch and retrapping is described in detail in chapter 3 .

Physically, the atom chip is an L-shaped solid copper block with outer dimensions of $11 \mathrm{~cm} \times 14 \mathrm{~cm} \times 18 \mathrm{~cm}$. The varnish-isolated copper wires are mounted with heat conducting glue in CNC milled grooves on the outer side of the atom chip. They have a cross section of $1.5 \mathrm{~mm} \times 2.5 \mathrm{~mm}$. The perpendicular wires are located underneath the longitudinal guide wires. The atom chip is split in two parts to accommodate for an additional supply line for the atom guide approximately half-way to the retrapping area. This enables independent current control in the front and back thus ensuring the simultaneous operation of both areas. The atom chip is mounted outside the vacuum in an L-shaped flange (see Fig. A.2). It is divided from the inside of the vacuum chamber only by a $500 \mu \mathrm{m}$-thick steel foil. 


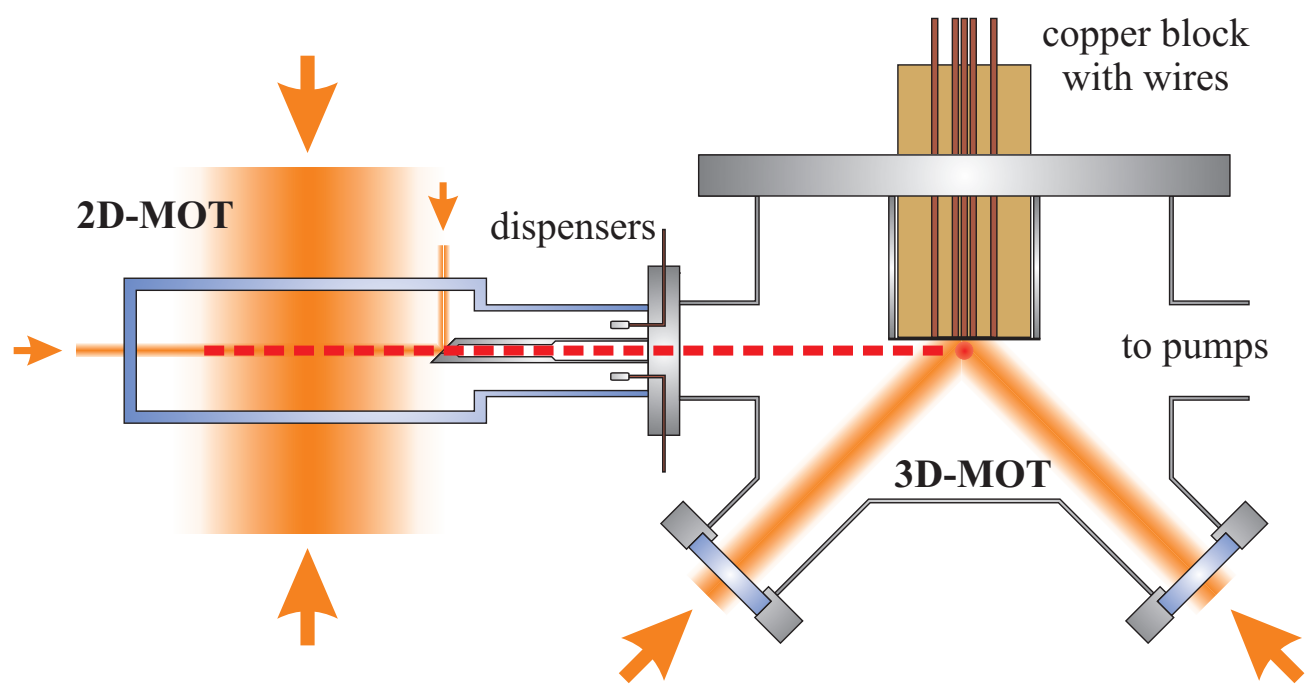

Figure 2.11.: Double MOT system. A coil-based 2D-MOT loads a 3D-MOT on a mesoscopic atom chip. High-power beams and large trapping volumes are key to this high-flux atom source.

\subsection{Double MOT system}

Preparing large atomic ensembles in sufficiently short time demands a high-flux atom source. Here, we chose a double-MOT system combining a 2D-MOT and a 3D-MOT. The basic working principle of a magneto-optical trap is presented in Chapter 1.4.3. The 2D-MOT is the initial atom source. In this stage, dispensers fill a vacuum glass cell with rubidium vapor at a sufficient partial pressure to operate a MOT from background vapor. The 2D-MOT chamber is connected to the main chamber through a differential pumping stage. The two-dimensional magnetic quadrupole field is generated by four $12 \mathrm{~cm} \times 17 \mathrm{~cm}$ rectangular coils with 81 windings each and a current of $4.5 \mathrm{~A}$ in each coil. The trap center is movable by adjusting the coil currents to guide the beam through the pumping stage. The light fields are sent to the experimental chamber in two separate fibers, they are then polarized by a quarter-wave plate and elliptically broadened in a telescope, resulting in two elliptical beams with a diameter of $87 \mathrm{~mm} \times 30 \mathrm{~mm}$ and a power of $200 \mathrm{~mW}$ each. They contain cooling light as well as repumping light (see Fig. B.1). The beams are passed through the 2D-MOT cell and are reflected back to the same path, thus forming a 2D-MOT with the magnetic field in the center of the glass cell.

Two additional beams are used to enhance the performance of the 2D-MOT. One is the so called pushing beam, pointing in the desired direction of the atom beam, accelerating the atoms towards the 3D-MOT. The other is the retarding beam, reflected off the differential pumping stage. The differential pumping stage has a 
polished, $45^{\circ}$-angled tip for this purpose. The central hole in the pumping stage imprints a ring shape on the reflected beam, so that the retarding beam decelerates atoms not cold enough to stay in the trap center. Both overlapping beams have a diameter of $5 \mathrm{~mm}$ and carry a fraction of the light for the radial cooling beams. The combination of a conventional 2D-MOT with a pushing and retarding beam is called $2 \mathrm{D}^{+}$-MOT and enables a larger flux, a lower mean longitudinal velocity and a smaller width of the velocity distribution [80,107]. The lower bound for the flux determined by the initial loading rate of the $3 \mathrm{D}$-MOT is $8.8 \times 10^{10}$ atoms $/ \mathrm{s}$.

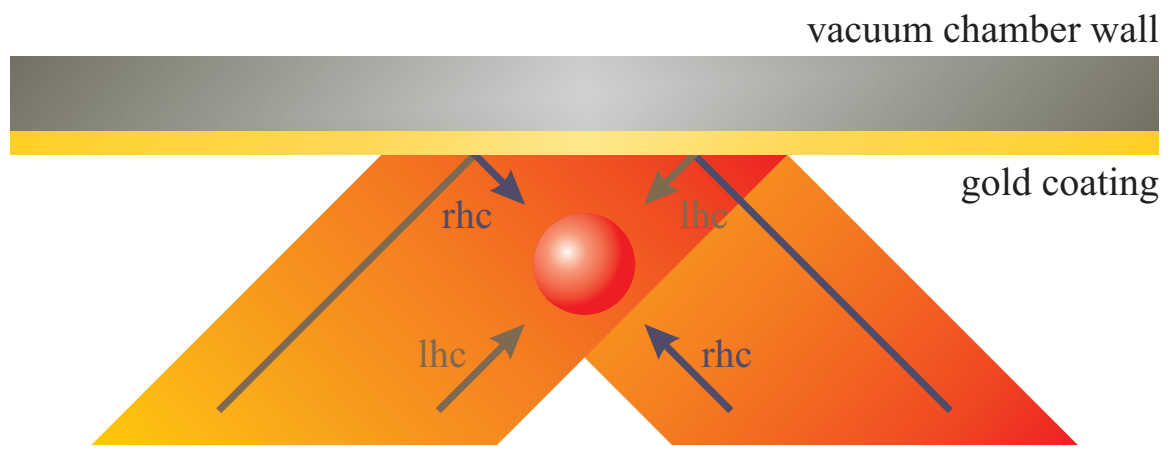

Figure 2.12.: Schematic drawing of a mirror-MOT. Four beams from a typical MOT configuration are replaced by two beams reflected by a common surface. The left-/right-handed circular polarization (lhc/rhc) matches the orientation of the magnetic quadrupole field.

The atom beam prepared by the 2D-MOT is send through the differential pumping stage and loads the 3D-MOT. The three-dimensional, magnetic quadrupole field for the 3D-MOT is generated by nine wires of the mesoscopic atom chip (see Chap. 2.7), with its minimum $7 \mathrm{~mm}$ below the vacuum wall. The 3D-MOT light is led by two fibers to the experimental chamber. One contains cooling light for the horizontal MOT-beam, which is reflected by a mirror coated with a quarter-wave plate. The beam is collimated to a diameter of $15 \mathrm{~mm}$ and circularly polarized. The other fiber carries cooling and repumping light which is collimated to $22 \mathrm{~mm}$. It is then split into two beams which are shone into the vacuum chamber from below in a $45^{\circ}$-angle. They are reflected off the underside of the L-shaped flange housing the atom chip. This surface is gold coated to maximize the reflectivity. The trap center forms where the incident and the reflected beams overlap in a mirror-MOT configuration [108] (see Fig. 2.12). This is a solution to the limited optical access that arises from the atom chip blocking one side of the trap. The power of the beams in the 3D-MOT is $90 \mathrm{~mW}$ for the horizontal beam and $340 \mathrm{~mW}$ for both $45^{\circ}$-beams combined. The $3 \mathrm{D}$-MOT loads $1.5 \times 10^{10}$ atoms within $300 \mathrm{~ms}$ with an initial loading rate of $8.8 \times 10^{10}$ atoms/s. This particularly good performance is due to the combination of benefits from the 2D-MOT and the 3D-MOT. 
The 2D-MOT is efficiently loaded from a relatively large-pressure background gas and led through a differential pumping stage. Here, the 3D-MOT is in a region with good vacuum conditions and thus very little losses due to background gas collisions. Additionally, the trapping volume of a 2D-MOT is much larger than that of a 3D-MOT, leading to higher loading rates. Compared to other beam sources with similar performance, such as Zeeman slowers (see Chap. 1.4.1), the 2D-MOT is an especially compact setup.

\subsection{Molasses and state preparation}

The atoms in the described 3D-MOT have a temperature of $130 \mu \mathrm{K}$ and are statistically distributed over the available spin states (see Chap. 2.6.2). Three steps are taken to prepare the atoms for further trapping or guiding with low losses: Compression and transfer of the MOT, optical molasses cooling and state preparation through optical pumping.

The first step is to compress the MOT and move it closer to the chip. The original MOT at a position $9.4 \mathrm{~mm}$ from the guide wire center features an undistorted quadrupole field, a large trapping volume and low losses. This MOT is pushed closer to the chip surface with a new trapping distance of $3.4 \mathrm{~mm}$ and forms a compressed MOT [109] to increase its density and to match its shape to the magnetic trap or guide. The compression is achieved by adjusting the currents to roughly double the gradients on the three axes of the cooling light to $(19,34$, 16) $\mathrm{G} / \mathrm{cm}$ and by also doubling the detuning to $-9.0 \Gamma$. The lifetime in the compressed MOT is significantly lower than in the relaxed MOT due to light induced losses in the compressed state as well as the close distance to the vacuum wall, where collisions lead to losses. With $900 \mu \mathrm{s}$ the time of the compression phase is kept relatively short to avoid these losses.

In the second step, the atoms are cooled in a short molasses phase. The small size and low trap depth of both the magnetic trap and the guide make this additional cooling step especially beneficial. The atoms are cooled in an optical molasses based on laser polarization gradient cooling [110]. For the molasses, the MOT laser beams are further red-detuned while no magnetic field is present. In the optimal case, rubidium 87 atoms can thus be cooled to temperatures as low as a few microkelvin. Two special properties of the experiment limit the efficiency of the process. One is the power-imbalance in the incoming and reflected beams for the optical molasses due to a non-perfect reflectivity of the gold coated vacuum wall. This leads to incoming beams with a higher power than the reflected beams and the atoms are pushed towards the vacuum wall. The uneven surface also leads to wavefront distortions of the reflected beam. The other limitation is a residual magnetic field after the currents are switched off. Even though the switch-off time of the currents is only a few $100 \mu \mathrm{s}$, the magnetic field still persist for several milliseconds due to eddy currents in the copper base of the mesoscopic atom chip. Future 
versions of the atom chip could reduce eddy currents significantly by choosing a base material that is non-conducting (see Chap. 5).

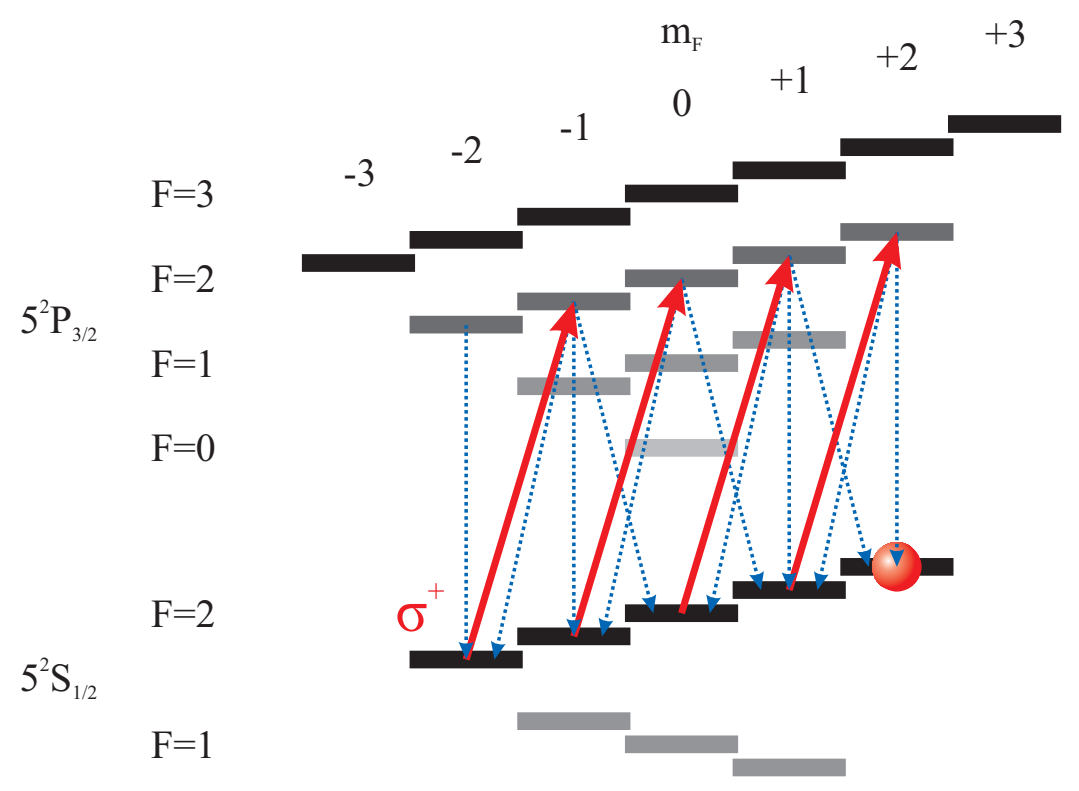

Figure 2.13.: Optical pumping process. Schematic drawing of the repeated absorption of $\sigma^{+}$-polarized photons (red) and spontaneous emission (blue). The atoms are transferred to the $\left|F=2, m_{F}=2\right\rangle$-state after a few absorbed photons.

The third and final step is to prepare the atoms in a desired state that is magnetically trappable. The potential that the atoms experience in the individual Zeeman levels depends on the magnetic quantum number $m_{F}$ (see Chap. 2.6.2). The strongest magnetic trapping potentials can be reached when the atoms are in the state $\left|F=2, m_{F}=2\right\rangle$. Initially, the atoms are statistically distributed over the two hyperfine states and their Zeeman sub-states after the molasses phase. A phase with a duration of $1 \mathrm{~ms}$ with only repumping light and no cooling light transfers the atoms into the $|F=2\rangle$-state (see Fig.B.1). Then, the atoms are transferred to the $\left|F=2, m_{F}=2\right\rangle$-sub-state by optical pumping. To this end, a homogeneous magnetic field with about $40 \mathrm{G}$ is generated by strong, counter-propagating currents in the outer perpendicular wires $p_{1}$ and $p_{4}$ (see Fig. 3.2). This field defines a quantization axis and lifts the degeneracy of the $m_{F}$-states. A $\sigma^{+}$-polarized light pulse with a duration of $1 \mathrm{~ms}$ enters from below and drives the optical pumping process illustrated in Fig. 2.13. The absorption of a $\sigma^{+}$-polarized photon increases the quantum number $m_{F}$ by 1 . Even though the spontaneous re-emission of the photon can change $m_{F}$ by \pm 1 and 0 , the directed change of the absorption quickly brings the atoms to the $m_{F}=2$-state. This state is a dark state for $\sigma^{+}$-polarized light and the pumping process stops. Now, the prepared atoms can be loaded 
into a three-dimensional magnetic potential, where $2.2 \times 10^{9}$ atoms are trapped. Alternatively, the atoms are confined and launched in a two-dimensional magnetic guide to form a continuous beam described in the next chapter. 



\section{Continuously pumped reservoir of ultracold atoms}

This chapter describes the first realization of a continuous cooling scheme in the microkelvin regime without the application of laser light. The general idea is based on a proposal for a magnetic trap that is continuously loaded by a pre-cooled atom beam [30].

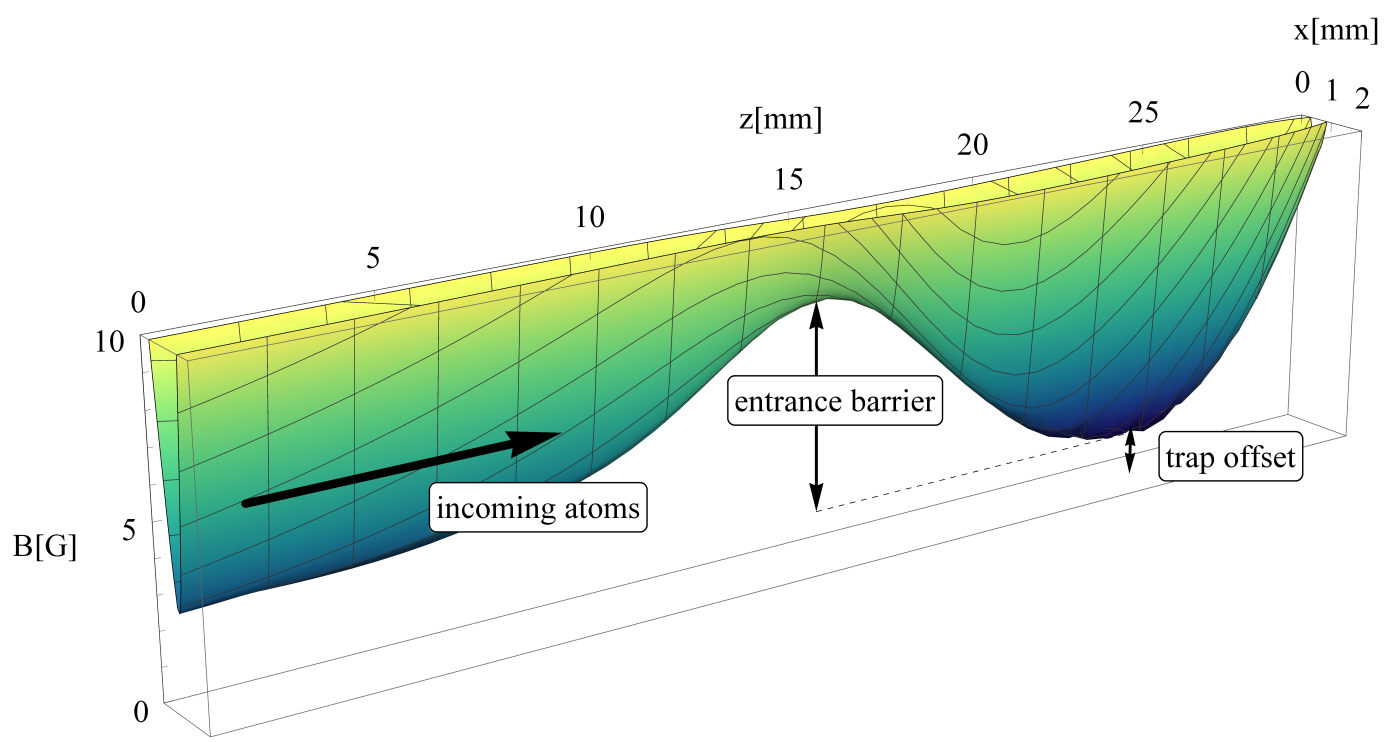

Figure 3.1.: Three-dimensional plot of the magnetic trapping potential. The potential is plotted in the $\mathrm{x}$-z-plane with the $\mathrm{y}$-coordinate fixed to the point of the trap minimum (see Fig. 3.2 for orientation regarding the mesoscopic atom chip). The atoms enter from the left and pass the entrance barrier. They undergo collisions in the trap and those losing enough energy are trapped.

Atoms are guided onto an entrance barrier of an highly elongated magnetic trap with a finite perpendicular trap depth larger than the entrance barrier (see Fig. 3.1). Those atoms passing the entrance barrier follow the elongated potential and are reflected at the end of the trap or undergo collisions along the way. A strong radial confinement ensures that these collisions take place for most atoms before they reach the entrance barrier again. The collisions allow for a redistribution 

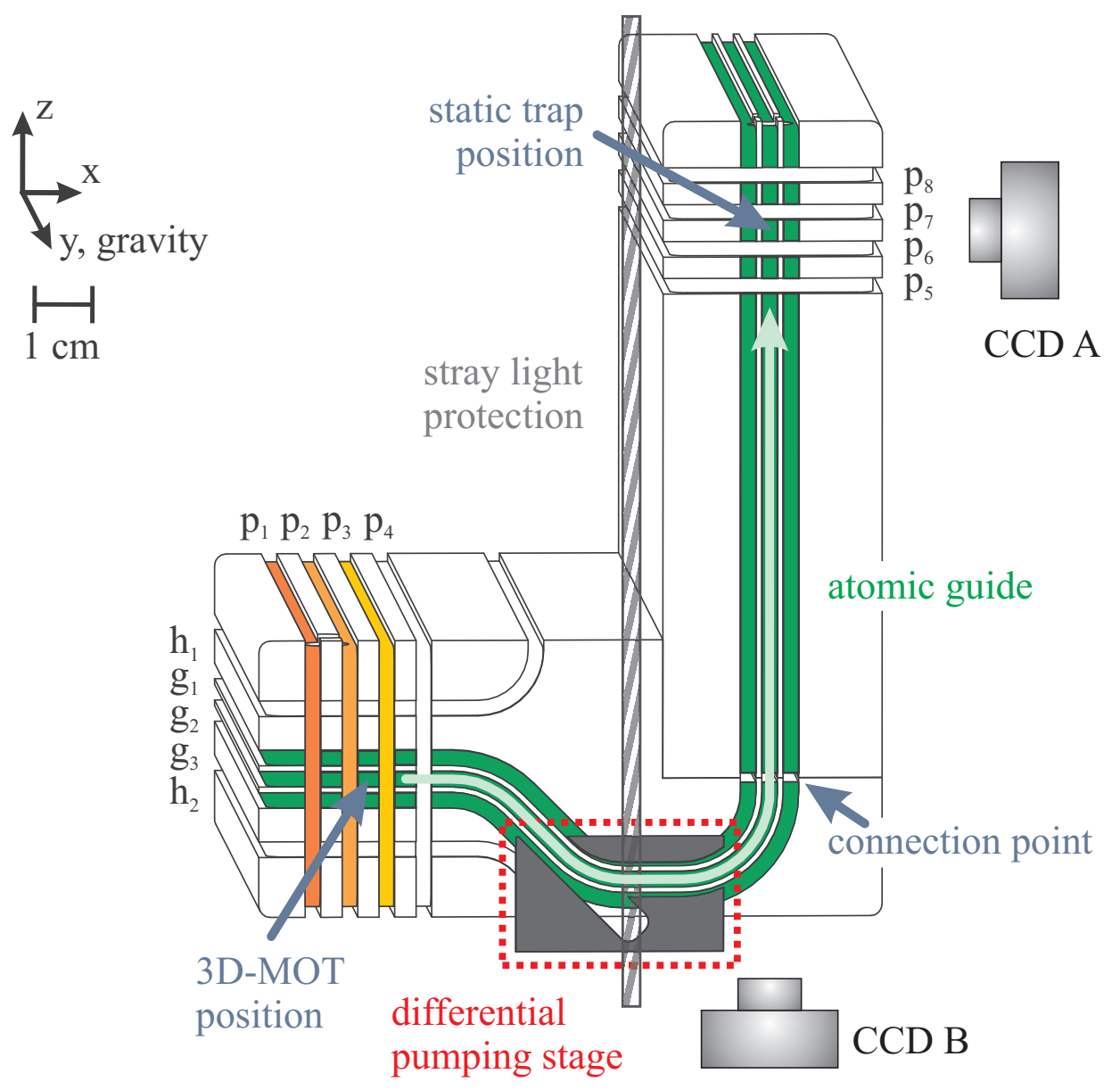

Figure 3.2.: Schematic drawing of the mesoscopic atom chip. The 3D-MOT is placed in the lower left area of the chip and is connected with the static trap position by an atomic guide (green). The guide passes a differential pumping stage in a vacuum divider (gray striped), which also shields the static trap from stray light. The wires are labeled as guide wires $g_{i}$, perpendicular wires $p_{i}$ and wires for compensation of hexapole components $h_{i}$. At the end of the guide, atoms can be detected at the static trap position from two directions with CCD cameras. Each guide wire features an additional perpendicular lead at the connection point, which separates the wire allowing for different currents through the front and rear guides. Therefore, the atom chip is assembled from two bulk copper blocks that hold and cool the wires. 
of kinetic energy. Some atoms gain energy such that their total energy is larger than the perpendicular trap depth and thus leave the trap. Other atoms will lose energy during the collisions such that their total energy is smaller than that of the entrance barrier and they will be trapped. These atoms will further enhance the collision probability for following atoms due to the enhanced density. A steadystate with a surprisingly large phase-space density forms with the right choice of trap parameters.

In this chapter, our experimental realization [111] of this proposal is presented. The different aspects are described in the order of the experimental cycle (see Fig. 3.2). First, the pre-cooled atoms from the 3D-MOT are prepared (see Chap. 2.8 and 2.9) and launched into the guide (Chap. 3.1). Then, the elongated trap is explained (Chap. 3.2), followed by a description of the continuous loading process (Chap. 3.3). Chapter 3.4 presents an extension of the setup by a supplementary optical dipole trap.

\subsection{Preparation of the atomic beam}

One main part of the continuous trapping scheme is the atomic beam. The thorough characterization of the beam is essential to produce a fitting trapping potential. After the 3D-MOT and state preparation, $1.3 \times 10^{9}$ atoms in the $F=2, m_{F}=$ 2 state can be loaded into a quadrupole trap. For the atom beam these atoms are not transferred to the quadrupole trap but directly enclosed and launched into an atomic guide. The magnetic guide is generated by the three inner wires $g_{1}$ to $g_{3}$ with static currents of $I\left(g_{1}\right)=105.3 \mathrm{~A}, I\left(g_{2}\right)=-102.5 \mathrm{~A}$ and $I\left(g_{3}\right)=118.3 \mathrm{~A}$. These currents are switched on quickly after state preparation and the atoms are trapped in two dimensions. The atomic cloud is launched into the guide by a sequential, linear increase of the currents in the perpendicular wires $p_{1}$ to $p_{3}$ (see Fig. 3.3). The sequence was optimized with the automated experimental optimization (see Chap. 2.5.3).

The atoms travel in the guide towards the static trap position. On their way, they pass the differential pumping stage that is marked in red in Fig. 3.2 which separates the two vacuum areas for better vacuum conditions in the retrapping zone. It also shields the retrapping area from unwanted stray light from the 3DMOT to enable simultaneous operation in both areas.

For an analysis of the beam characteristics, the atoms are detected with an absorption detection on two cameras (see Fig. 3.2) once they arrive at the static trap position. The cameras operate with charge-coupled devices (CCD). One detection direction is perpendicular to the atomic guide (CCD A) and one is along the guide (CCD B). This detection system is used to characterize the atomic beam.

The beam velocity is measured by launching an ensemble of atoms and detecting its arrival at different positions within the $15 \mathrm{~mm}$ wide detection area of CCD camera A. The results of such an experimental evaluation are plotted in Fig. 3.4 


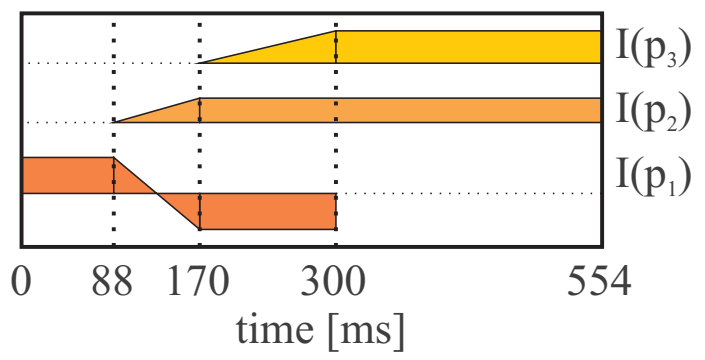

Figure 3.3.: Sequence for the launch of an atomic cloud. Currents and timings that are used to accelerate the atoms in the guide. The peak currents are $I\left(p_{1}\right)= \pm 103.0 \mathrm{~A}, I\left(p_{2}\right)=69.8 \mathrm{~A}$ and $I\left(p_{3}\right)=92.6 \mathrm{~A}$. The colors match those of Fig. 3.2.
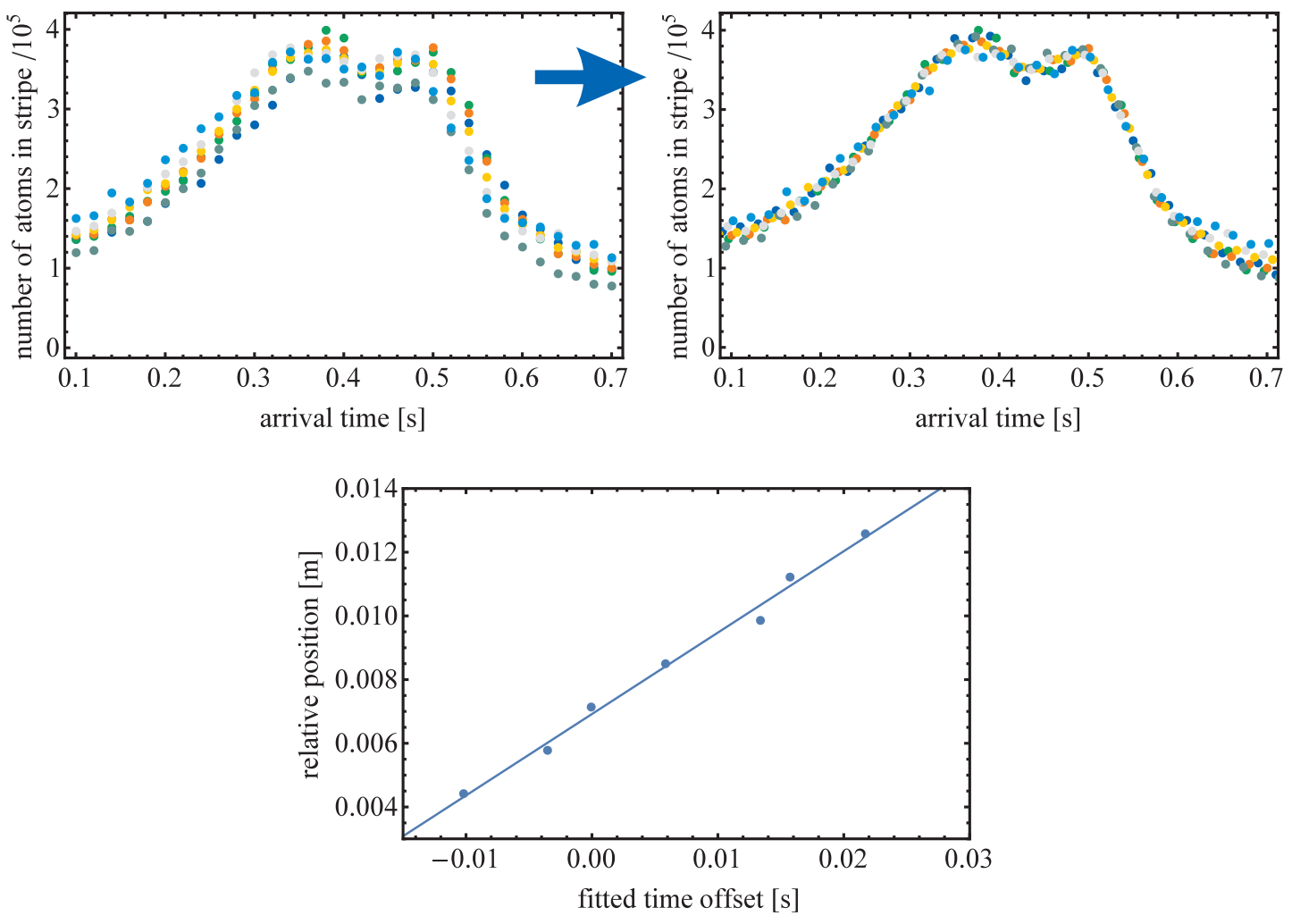

Figure 3.4.: Beam velocity measurement. Top left: Experimental evaluation of the different arrival times at different positions in the detection zone. The colors represent a different detection position. Top right: The amplitude and time offsets are fitted to minimize the pairwise squared difference of the interpolated data. Bottom: The velocity is inferred from the results of the fit and the position difference. 
in the top left corner. The different colors correspond to the different positions of detection. The double peak profile is a result of the three-step acceleration process. The curves are brought to a match afterwards with only two parameters: One to adjust the arrival time and the other to correct small detection efficiency imbalances in the total amplitude of the curve (see top right plot). A linear fit of the relative position over the correction for the arrival time results in a mean velocity of $25.6 \mathrm{~cm} \mathrm{~s}^{-1}$ (see bottom plot). This velocity is adjustable with the timing and strength of the launch currents. It is chosen such that it balances losses during the launch and kinetic energy brought into the static trap while maintaining a high flux.

A velocity distribution is inferred from the same data as presented in Fig. 3.4 by taking the total traveling distance of $20 \mathrm{~cm}$ into account. The distribution is plotted in Fig. 3.5. Integrating the distribution yields a total number of $8.4 \times 10^{7}$ atoms. A longitudinal temperature of $93 \mu \mathrm{K}$ can be obtained from the width of the distribution. The radial temperature of $77 \mu \mathrm{K}$ is measured by monitoring the ballistic expansion in the trapping region.

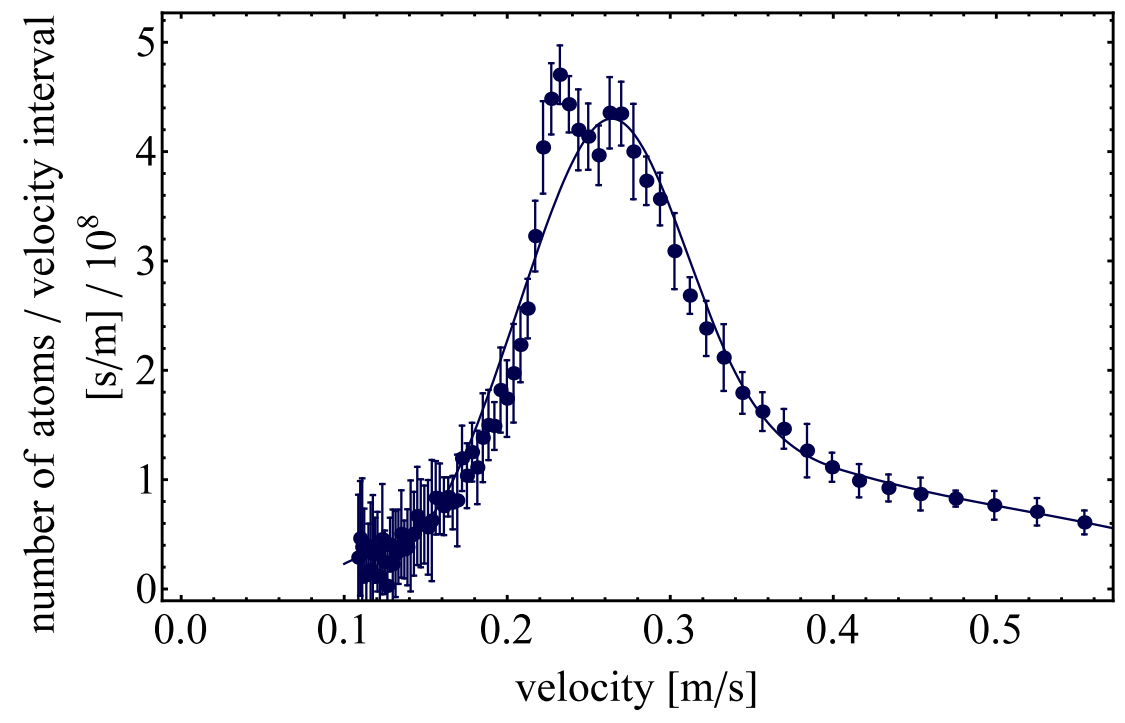

Figure 3.5.: Velocity distribution of the beam. The longitudinal beam temperature can be inferred from the width of the distribution and the total number of atoms from an integration of the curve.

The guide currents in the MOT and retrapping area are controlled separately for a repeated operation. After the atoms cross the connection point of the two areas (see Fig. 3.2), the next cloud is prepared in the MOT region and launched into the guide. The launched atom clouds spread out in the guide and overlap, forming a continuous atom beam with modulated amplitude. A cycle time of $1.1 \mathrm{~s}$ results in a total average flux of $7.6 \times 10^{7}$ atoms s $^{-1}$. 


\subsection{Elongated trapping potential}

Based on the characterization of the atom beam, a trapping potential can be created according to the proposal [30]. The mesoscopic atom chip is capable of creating the magnetic potential for the trap. Here, the atomic guide (created with wires $g_{1}$ to $g_{3}$ ) is used for the radial confinement. The wires $p_{5}$ to $p_{8}$ (see Fig. 3.2) are used to complete the three-dimensional trap and adjust the relevant trap parameters: The entrance barrier height, the trap offset and the longitudinal trap frequency. The optimal trapping potential is obtained via a thirteen dimensional optimization with the automated computer control. The optimal currents for the static trap are $I\left(p_{5}\right)=28.1 \mathrm{~A}, I\left(p_{6}\right)=0.5 \mathrm{~A}, I\left(p_{7}\right)=-3.9 \mathrm{~A}$ and $I\left(p_{8}\right)=70.9 \mathrm{~A}$.

A simulation of this potential is shown in Fig. 3.1. The magnetic fields are simulated utilizing a numerical integration of Biot-Savart's law. The influence of the supply wires is included in the calculation and we confirmed that the wires can be well approximated by infinitesimal conductors at the relevant distances. The simulations are verified by comparing the most sensitive results to the experiment: The trap offset in the center of the trap and the trap frequencies in the three main directions.

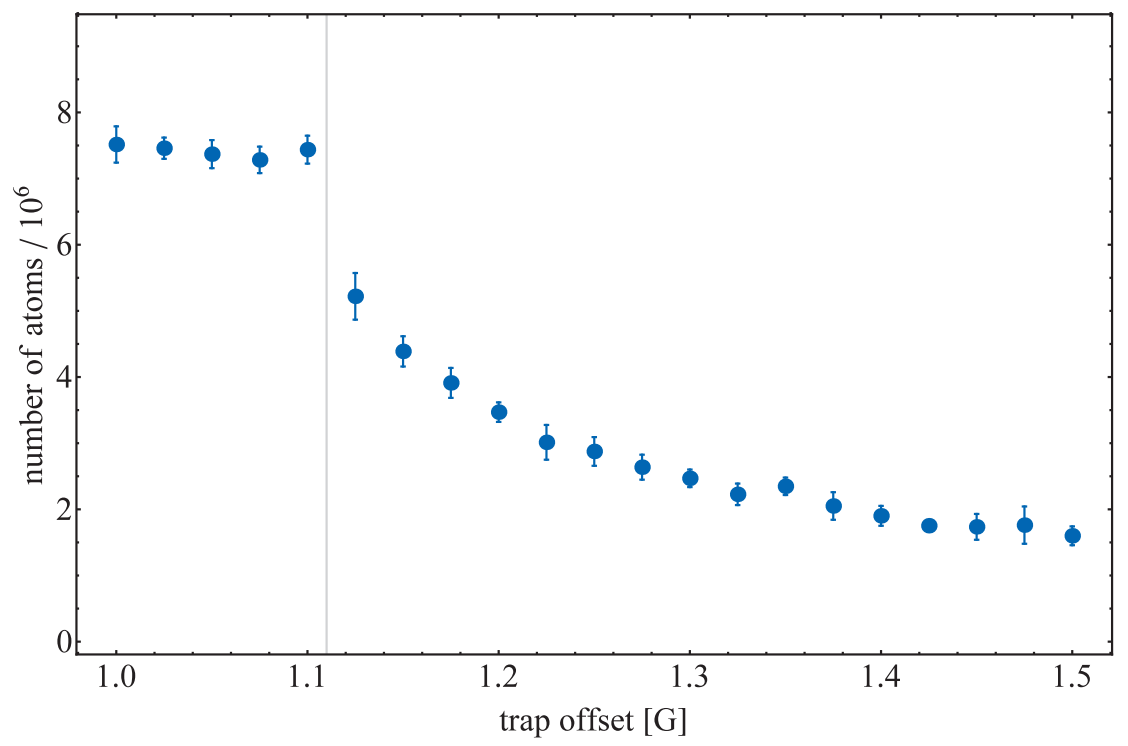

Figure 3.6.: Trap offset determined by microwave spectroscopy. The gray vertical line indicates the fitted trap offset of $1.11 \mathrm{G}$.

To measure the trap offset, an evaporatively cooled ensemble in the $F=2, m_{F}=$ 2 state is loaded into the trap. Afterwards, microwave spectroscopy is performed on the transition to the $F=1, m_{F}=1$ state. The results are plotted in Fig. 3.6. Atoms are lost from the trap if the detuning of the microwave matches the potential energy difference of the trapped atoms to the $F=1, m_{F}=1$ state. If the 
microwave detuning is chosen such that the energy is not sufficient anymore to expel atoms from the trap, the trap offset is reached. A fit to the spectroscopy data results in an offset field of $1.11 \mathrm{G}$ which is in excellent agreement with the calculation resulting in $1.15 \mathrm{G}$.

The trap frequency measurement also starts by loading the trap with evaporatively cooled atoms. A small but rapid disturbance in the magnetic field is used to start an oscillation of the cloud in the trap. Detecting the atoms after a long final ballistic expansion with CCD cameras A and B resolves this oscillation in the three main directions. The result of the experiment is plotted in Fig. 3.7. The resulting trap frequencies are $\omega_{x, y, z}=2 \pi \times(171,168,5.6) \mathrm{Hz}$. This is in good agreement with the results of the simulation $\omega_{x, y, z}=2 \pi \times(186,185,6.4) \mathrm{Hz}$. This trap features the desired elongation with an aspect ratio of 30 and can thus be used for the continuous loading scheme.
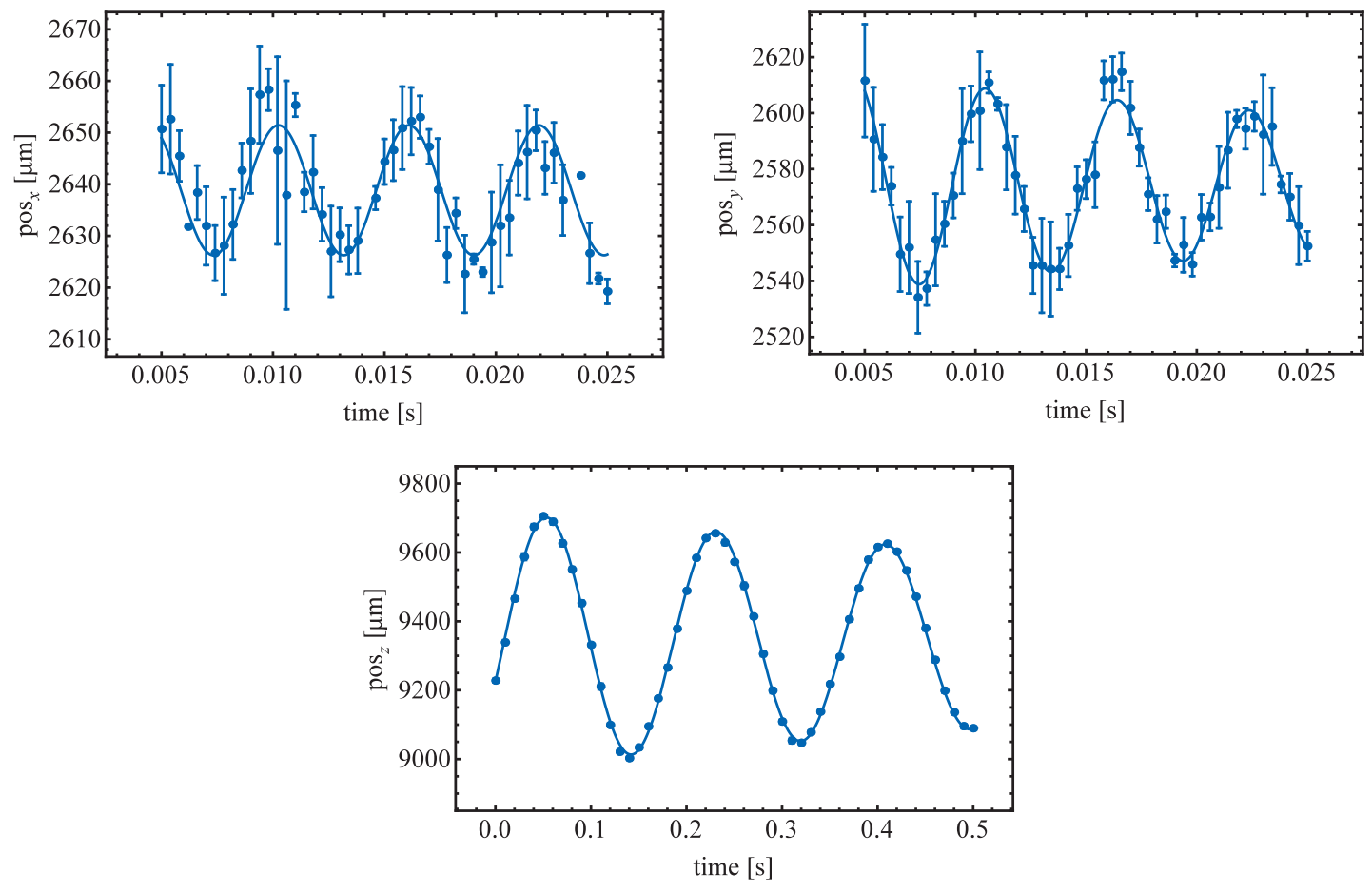

Figure 3.7.: Trap frequency measurements. The trap frequencies are measured in all three directions by disturbing a precooled atom cloud in the trap and observing its oscillations after a ballistic expansion. 


\subsection{Continuous loading}

The continuous atom beam created from repeatedly prepared and launched cold clouds is now guided to the static trap and the continuous loading mechanism is started. When atoms pass the entrance barrier of the magnetic trap, the atoms start to accumulate. Figure 3.8 shows the number of trapped atoms as a function of time. The atoms are detected after a holding time of $2.25 \mathrm{~s}$. This ensures, that only trapped atoms are counted. In the final steady state, there are $3.8 \times 10^{7}$ atoms trapped and the initial loading rate is $3.1 \times 10^{6}$ atoms $^{-1}$. As predicted by the proposal, the increasing number of atoms goes along with a decreasing temperature. This effect has been observed in ballistic expansion and is plotted in Fig. 3.9. The steady state temperature is at $102 \mu \mathrm{K}$.

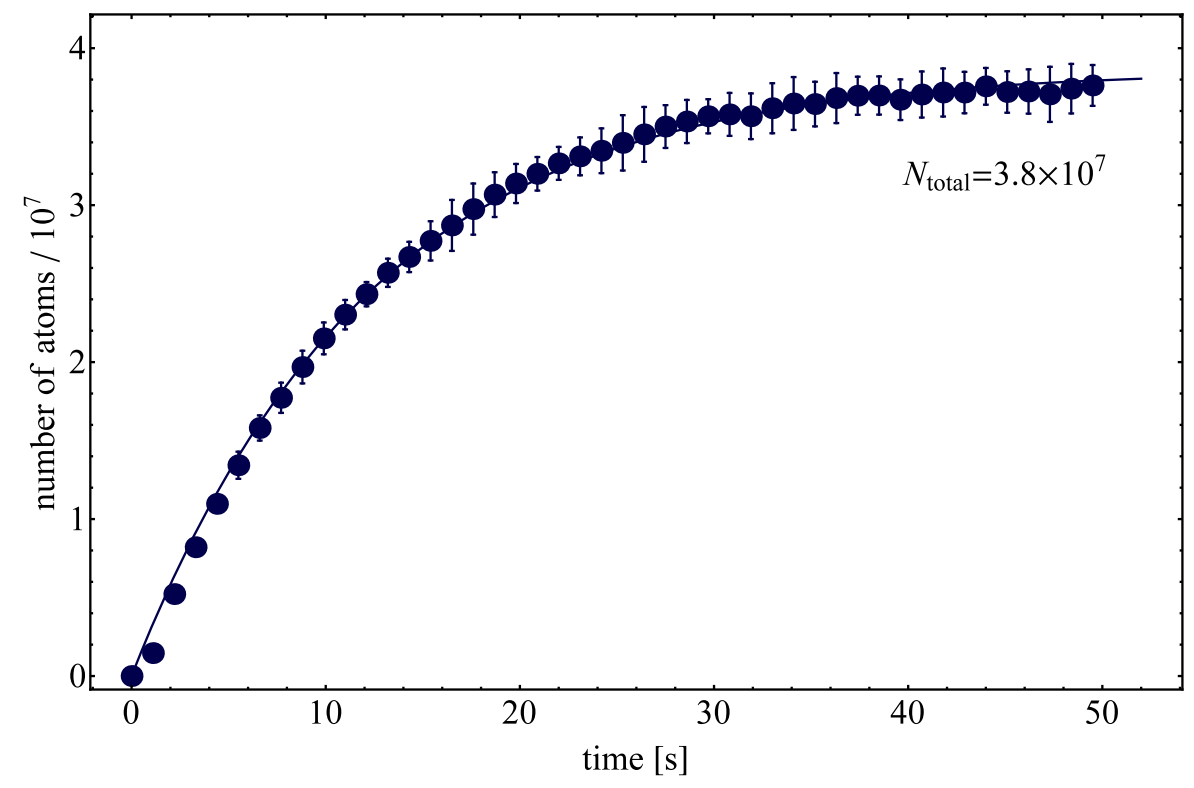

Figure 3.8.: Continuous accumulation of atoms in the static trap. The error bars represent the standard deviation of eight evaluated measurements.

A phase-space density in the trap of $9 \times 10^{-8} \mathrm{~h}^{-3}$ is calculated according to the formula

$$
\begin{aligned}
\mathrm{PSD} & =n_{\text {atoms }} \times \text { spatial density }{ }^{3} \times \text { momentum density }^{3} \\
& =n_{\text {atoms }} \times \frac{1}{(2 \pi)^{3 / 2} \cdot \sigma_{x} \cdot \sigma_{y} \cdot \sigma_{z}} \times \frac{1}{\left(2 \pi m k_{B} \cdot T\right)^{3 / 2}},
\end{aligned}
$$

with the number of atoms $n_{\text {atoms }}=3.8 \times 10^{7}$ atoms, the size of the trappedcloud $\sigma_{i}$ and the temperature $T=102 \mu \mathrm{K}$. The accumulation of ultracold atoms in a static and conservative trap presents a key result of this thesis. 


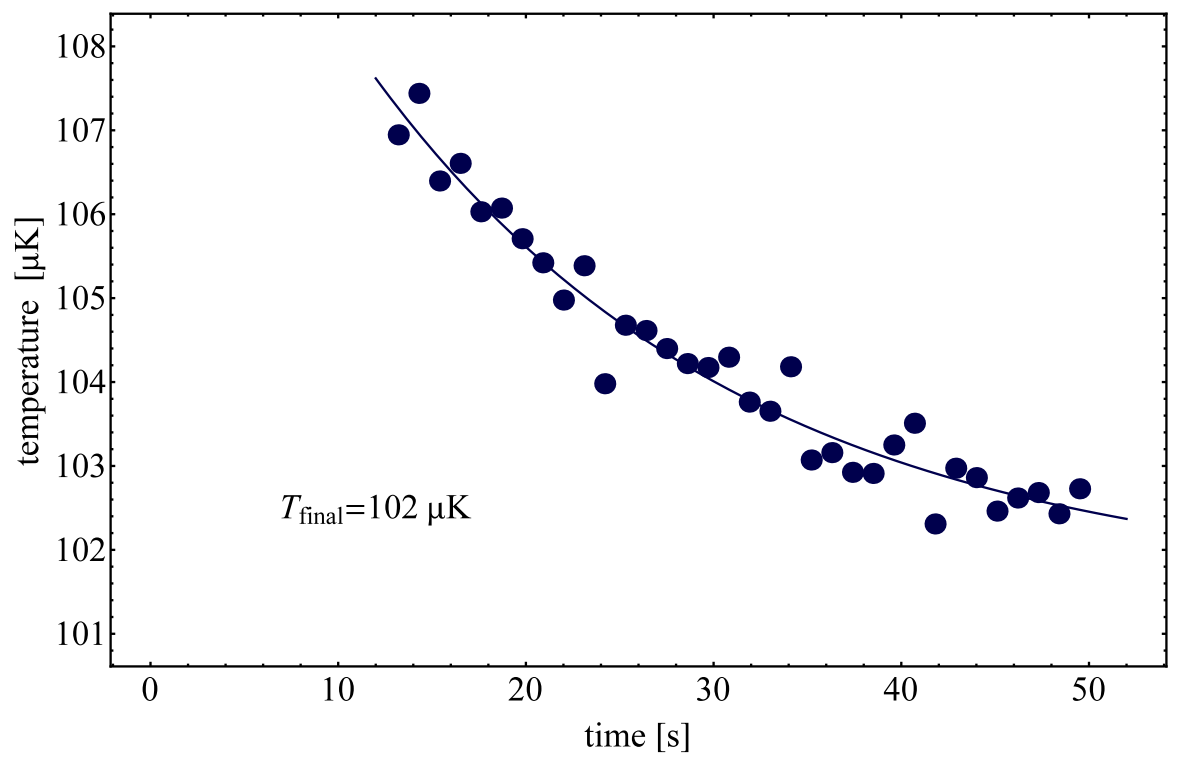

Figure 3.9.: Decreasing temperature during the continuous loading process. The decreasing temperature accompanies the accumulation of atoms in the static trap (see Fig. 3.8). The temperature is derived from time of flight images at $5 \mathrm{~ms}$ and $8 \mathrm{~ms}$. An exponential decay fit to the data suggests a final temperature of $T_{\text {final }}=102 \mu \mathrm{K}$ for loading times over $50 \mathrm{~s}$, while the number of atoms already saturates after $40 \mathrm{~s}$. 
The optimal parameters of the trap were found with the help of the automated experimental optimization (see Chap. 2.5.3). One crucial parameter is the entrance barrier height. A scan of the barrier height without further adjustments of the trap is plotted in Fig. 3.10. When the entrance barrier is too low, the atoms are likely to leave the trap even after energy losses through collisions. With a barrier that is too high, a large portion of the atoms is not able to enter the trap and the flux is reduced. The entrance barrier height fitting best to the kinetic energy of the beam is at $310 \mu \mathrm{K}$. This corresponds to $65 \%$ of the atoms entering the trap. The optimal barrier height is strongly linked to the forward velocity of the incoming beam. In principle, a decrease in the barrier height has the same effect as an increase in the forward velocity of the beam. This scenario would result in additional kinetic energy of the atoms in the trap, which could be compensated by an increase in the trap offset. With these adjustments, a wide range of beams can be accommodated for by the trap.

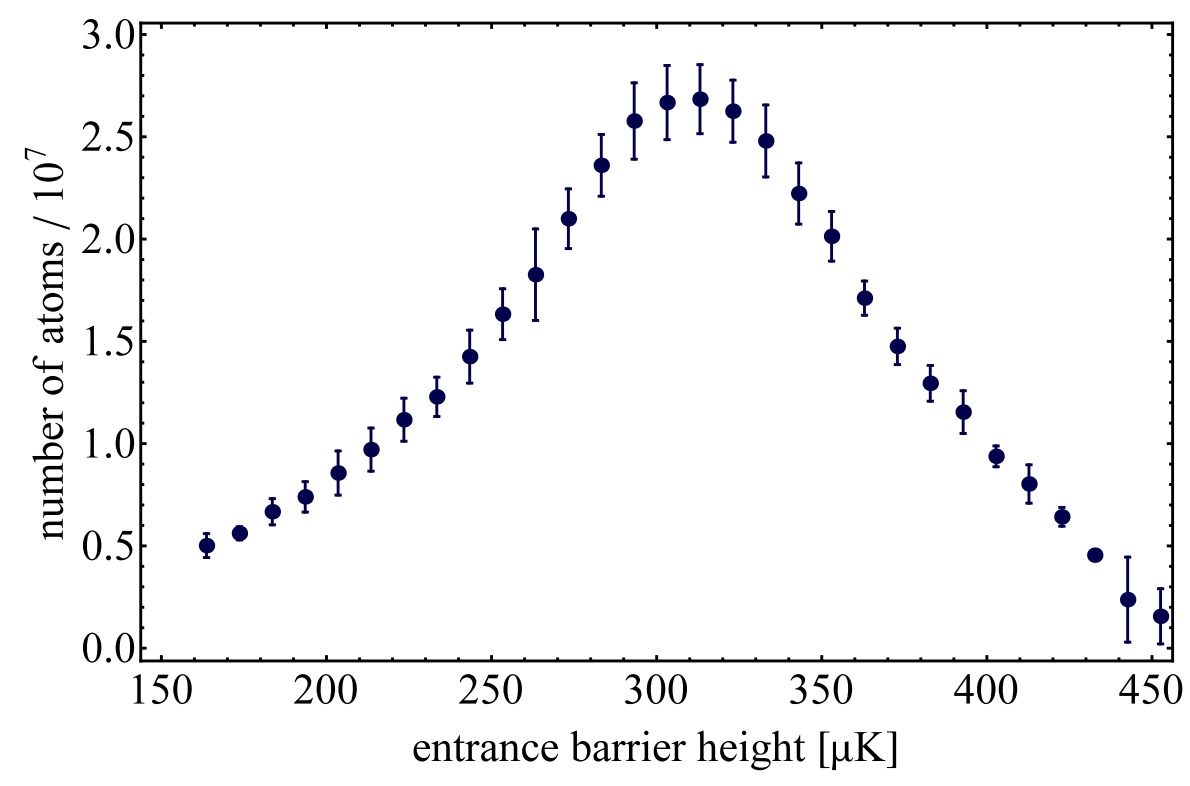

Figure 3.10.: Scan of the entrance barrier height. Number of accumulated atoms after a loading time of $33 \mathrm{~s}$. The error bars represent the standard deviation of four experimental realizations.

An efficient operation of the continuous loading scheme needs a transverse evaporation threshold to quickly eliminate hot atoms [30]. This threshold is naturally a part of the trap through the finite depth of the chip trap of $750 \mu \mathrm{K}$. This limitation arises due to technical current restrictions of the atom chip. Still, a further investigation of the influence of the trap depth was made by implementing an additional threshold: A forced microwave evaporation on the transition $F=2, m_{F}=2 \rightarrow F=1, m_{F}=1$. This microwave was switched on during an 
otherwise normal continuous loading of 33 s reducing the trap depth. A plot of the number of accumulated atoms with different trap depth is shown in Fig. 3.11. It is apparent that the number of atoms monotonously increases with an increasing trap depth. Since the number of trapped atoms does not saturate, the trap depth is identified to limit the overall performance.

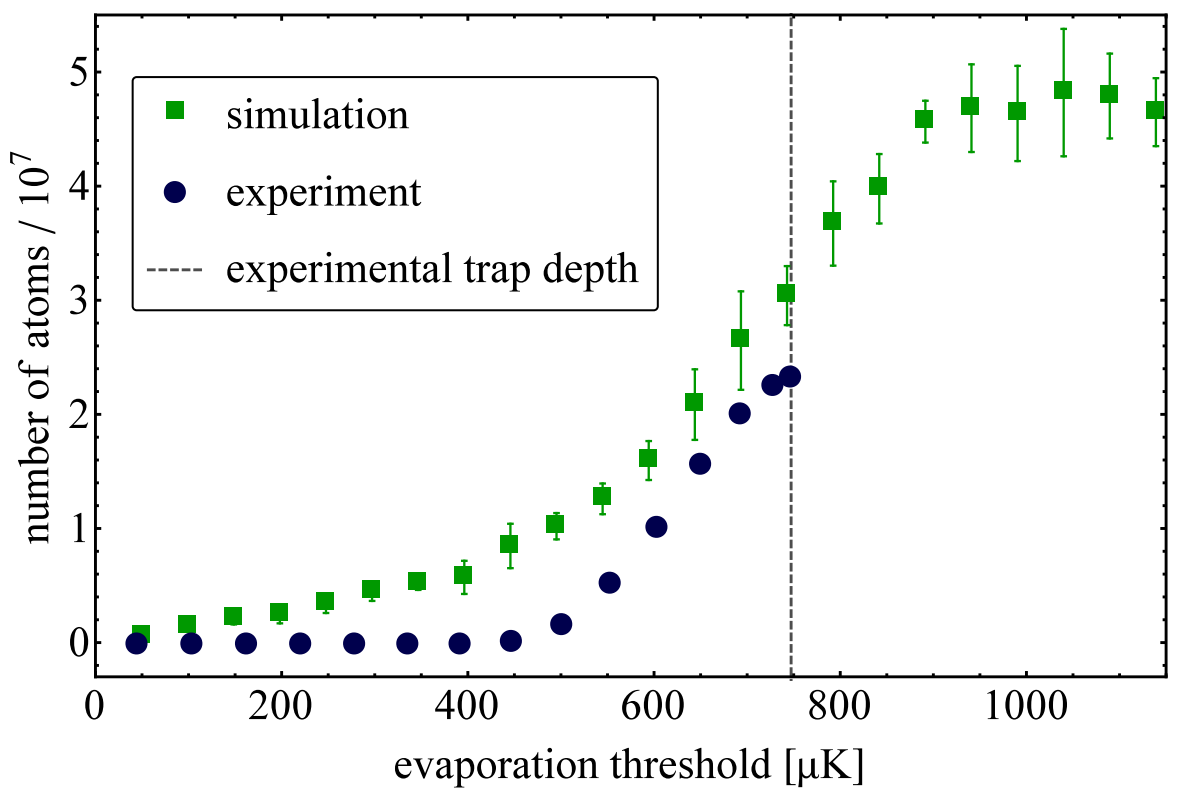

Figure 3.11.: Influence of the trap depth on the number of atom. The atoms are accumulated within $33 \mathrm{~s}$ of continuous loading. A microwave threshold limits the trap depth during the entire loading time. The blue circles are experimental data and the vertical, dashed line indicates the maximally realizable trap depth. The green squares are the result of a molecular dynamics simulation without a trap depth limitation but otherwise equal parameters.

To investigate this behavior, a molecular dynamics simulation according to [30] was used to estimate the potential increase of the atom number for deeper traps. These simulated data is also plotted in Fig. 3.11. According to the simulation, it is clear that the trap depth indeed limits the number of atoms but also that the achieved results are already quite close to the optimum. Thus, for an even further increase of the performance other factors also need to be considered, such as a tighter radial trapping confinement or an improved atomic beam flux.

The obtained results are only possible because of a long lifetime in the magnetic trap. In previous experiments with the apparatus, lifetimes of up to $240 \mathrm{~s}$ were observed, which is reachable due to a low background pressure in the retrapping region and a good stray light isolation. This lifetime is much larger than the relevant timescale of the continuous loading process. We investigated several factors 
that could limit the lifetime by operating the reloading process without atoms while holding previously accumulated atoms in the trap. By including and excluding parts of the loading sequence, their influence on the lifetime is detectable. Resonant laser light from the 2D/3D- MOT and external noise sources yield an additional loss of less than $20 \%$ after a holding time of $30 \mathrm{~s}$. However, switching the magnetic field for the atom beam creation has a far stronger influence, where we see a nearly exponential loss with a rate of $12 \mathrm{~s}$. This cross-talk of the two parts of the atom chip limits the beneficial loading time and thus the achievable number of atoms.

\subsection{Trap enhancement with a dipole beam}

An increase in the depth of the static trap and larger radial trap frequencies should enhance the performance of the loading process. Both aspects can be improved by superimposing an additional potential to strengthen the trap radially. This was investigated by adding an optical dipole trap potential atomic guide. The beam waist in the center of the trap. The setup consists of a fiber laser from NP Photonics (RFLM-50-1-1064-1) with an output power of $50 \mathrm{~mW}$ coupled to a fiber amplifier from Nufern (NuAMP SUB-1151-50) with an output power of $12 \mathrm{~W}$. The laser power is stabilized with a PID-controller. For this purpose, its light is monitored by a photodiode to create a feedback signal. The power is controlled with an acousto-optical modulator from Gooch $\& 5$ Housego (3110-197). The stabilization is also used to adjust the power of the dipole trap freely to a set point given by the computer control. This setup delivers light with a wavelength of $1064 \mathrm{~nm}$ and a power of up to $8 \mathrm{~W}$ to the experimental chamber.

A calculation of its effect on the trapping potential shows increased trapping frequencies and an increase in the maximal trap depth of more than $20 \%$. To find the optimal effect, the beam waist was adjusted over a wide range but for the calculation of the trap depth, a beam waist of $60 \mu \mathrm{m}$ is assumed. In addition, to the trap parameter change, the accumulation of atoms in the optical dimple should drastically increase the collision rates locally. One challenge of the setup is to achieve a good match of the magnetic trap and the optical dipole trap. The elongated vacuum chamber puts strong constrains on the possible angles for the dipole trap and the close vicinity of the atom chip over a long distance to the dipole trap limit the possibilities to decrease the waist for a stronger confinement.

Fig. 3.12 shows a false-color image of a continuously loaded cloud in the static trap with the optical dipole trap present during the entire loading time. The picture is taken in situ. The small peak in the middle of the density distribution is the result of the dipole trap. It is apparent that the dipole trap affects the magnetic trap and that atoms gather in it. To further support that actual trapping of atoms in the dipole trap occurs, the magnetic trap was switched off and remaining atoms in the dipole trap could still be observed. Unfortunately, even though there is a 


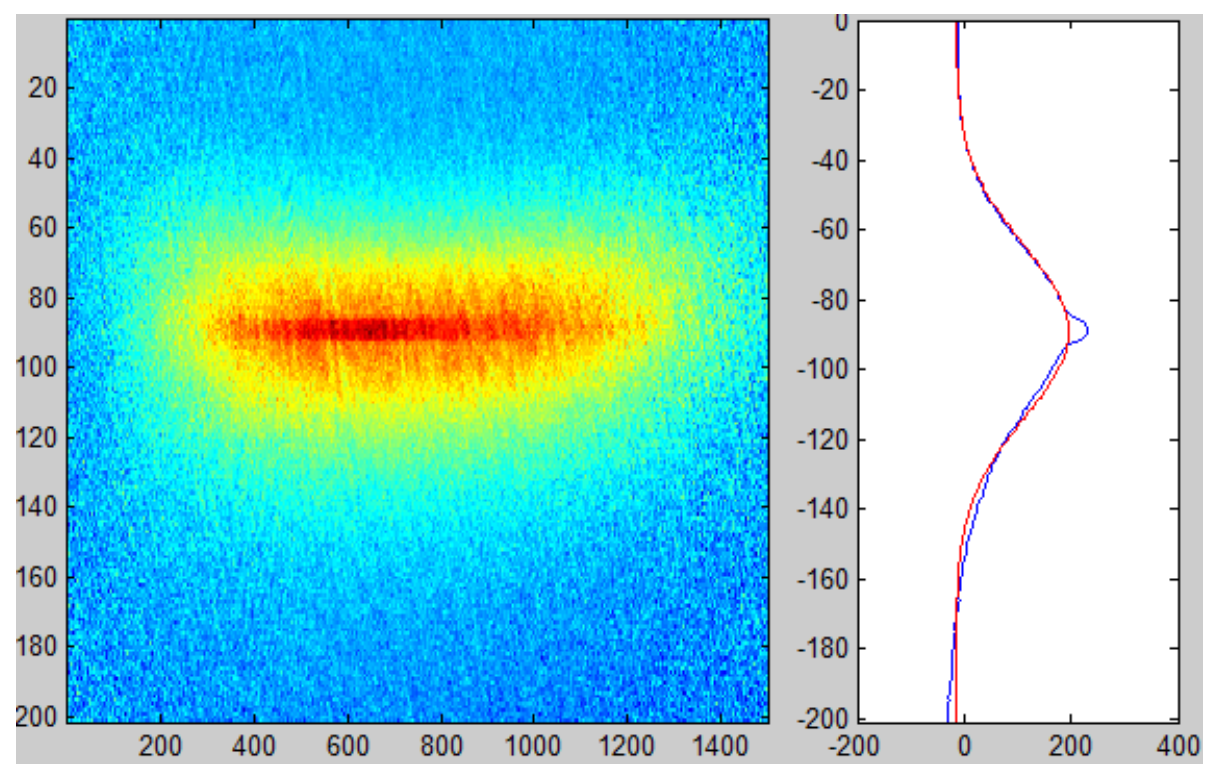

Figure 3.12.: False-color image of a continuously loaded cloud with dipole trap present in the center. The effect of the dipole trap is visible as small peak on the pixel sum.

visible effect on the trap and the loading rate increases by about $20 \%$, the final number of atoms in the trap did not increase. The most likely reason for the low performance of the added dipole trap is the comparably large temperature of the atoms in the trap. Due to this temperature, the atoms spend most of the time not in the trap center, where the dipole trap is present, but in the periphery without any influence of the dipole trap. The dipole trap thus does not contribute to enhanced collision rates, which would be the most beneficial effect for the continuous loading scheme. Consequently, a much stronger dipole trap should be implemented to truly collect the atoms in its trap center. 



\section{Microwave sidebands for laser cooling by direct modulation of a tapered amplifier}

\subsection{Compact laser systems}

In atom optics, cooling almost always necessitates the use of several laser sources. For example, the widely used alkaline earth atoms require two lasers for the operation of a magneto-optical trap. The atoms are cooled on a transition from one of the two hyperfine ground states to an excited state. This cooling transition is not completely closed and there is the possibility that the atoms decay from an excited state to the other hyperfine ground state. Here, they would remain without a second laser frequency bringing them back into the cooling cycle. The frequency difference of the two lasers equals the hyperfine splitting of the ground state and is in the range of several gigahertz. For instance, the ground state hyperfine splitting for sodium is $1.77 \mathrm{GHz}$, for rubidium $853.04 \mathrm{GHz}$, for rubidium $876.83 \mathrm{GHz}$ and for cesium $9.19 \mathrm{GHz}[59,112-114]$. The level scheme relevant for the cooling of rubidium 87 can be found in Fig. B.1.

In most cases, this ground state frequency difference is the reason to employ laser systems with two frequency-stabilized laser sources, like external-cavity diode lasers. Often, one of the lasers is stabilized via spectroscopy, the beat signal of the two lasers is then used to stabilize the second laser. To reach the necessary power of the lasers, they are typically amplified by a semiconductor laser amplifier, such as a tapered amplifier (TA).

Such a setup is reliable, flexible, already comparably low in power consumption and commonly used in many atom optics experiments including atom interferometry. This interesting field of research has lead to measurement devices with unprecedented precision (see Chap. 1.1). The measurement of quantities like rotation, acceleration, gravity and time independently of a stationary lab is highly desired and thus portable atom interferometers are developed. This need for mobility puts restrictions on reliability, power consumption, size and weight which are particularly strong for future space missions with applications from fundamental tests of general relativity to geodetic earth observations. In this context, the possibility to remove the need for one of the two cooling lasers is very tempting.

In principle, one of the lasers can be replaced by modulating the light of the 
other. For modulation frequencies up to one gigahertz, this can be well accomplished by standard modulation equipment like acousto- or electro-optical modulators. On the downside, these modulators add complexity and their efficiency drops significantly for large frequency shifts. To reduce the need for resources, it would be beneficial to generate the second frequency in already existing equipment. One possibility would be to directly modulate the current of one laser [115-117]. This modulation produces sidebands symmetrically around the carrier with a frequency offset equal to the modulation frequency. When using a diode laser with an external cavity, even the nearly complete suppression of the carrier frequency is possible by matching the external cavity free spectral range to the modulation frequency [118]. Modulating the laser diode current lets the same source emit two sidebands. This inherently makes the light of the carrier and the sidebands phase coherent and matches the spatial mode. Thus, this source is a convenient solution to generate Raman beams for state manipulation [119].

However, two disadvantages arise from the direct modulation of the master laser current. First, it is difficult to adjust the external cavity to accommodate two frequencies, and second, it is impossible to extract unmodulated light from the same source for manipulation and detection. This can be circumvented by modulating a seeded slave laser [120]. Unfortunately, without further components, the power of this setup is limited to typically $100 \mathrm{~mW}$. The drawbacks of the examples above can be avoided with a different method, the current modulation of a tapered amplifier. Three goals can be achieved by this modulation: Extraction of unmodulated light, few optical components and high output power. Such a modulation setup [121] was implemented, characterized and put to work at the experiment presented in this thesis [122].

\subsection{Modulating a tapered amplifier}

\subsubsection{Tapered amplifier introduction}

High laser power is especially important for the operation of a magneto-optical trap and thus for many atom optics experiments. One broadly used method to gain the necessary power is to amplify low-power light with the desired properties. In principle, a coherent light signal is injected into the active region of a semiconductor laser. It is amplified through induced recombination within the active medium maintaining a population inversion. The use of anti-reflection coating and the design of the active region help that the light basically maintains its spatial and spectral properties [123]. Among the various amplifier systems realized to date, the tapered amplifiers have been especially successful. Among the reasons are its small-signal high-gain amplification and good wave-guiding properties [124]. The basic shape of the active area of a tapered amplifier can be seen in Fig. 4.1. A short and straight active waveguide in the entrance area amplifies the low-power 


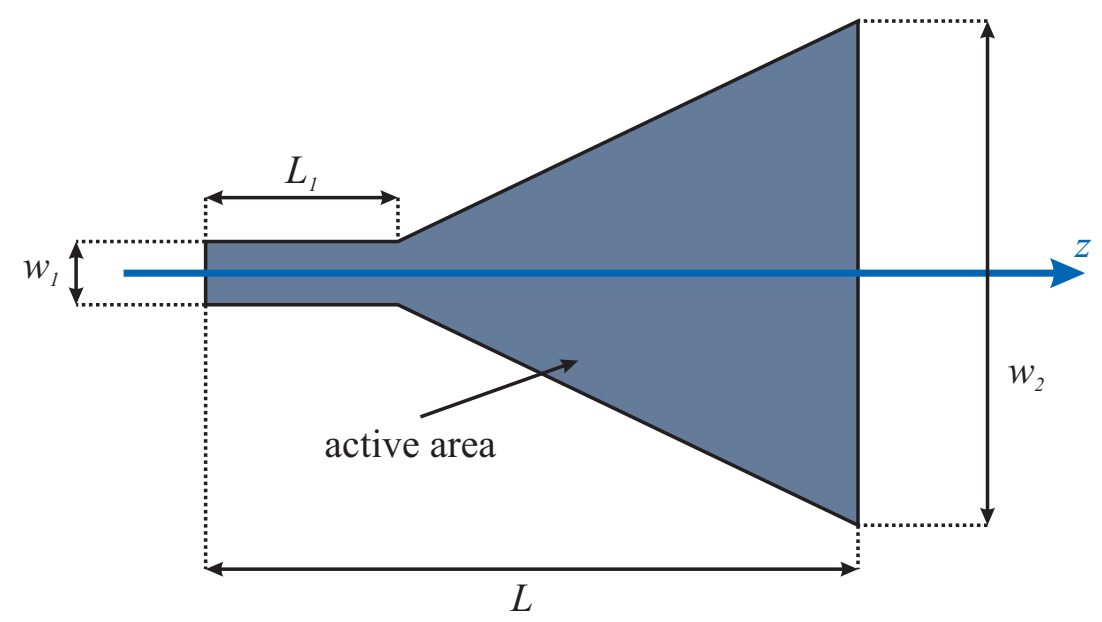

Figure 4.1.: Active area of a tapered amplifier. The light injected into the small waveguide on the left is distributed at the end of $L_{1}$ by diffraction into the tapered area. The large output facet $w_{2}$ enables a high output power at low intensities to avoid thermal damage to the facet.

input. Behind the waveguide, the beam is broadened by diffraction into the active, tapered area so that almost the entire area can be used for amplification. Typically, the length of the initial waveguide $L_{1}$ is a few hundred micrometers and the total length $L$ is around one to three millimeters [124]. The shape with the narrow initial guide as preamplification and the large tapered area enable a large gain from a small initial seed on the order of several ten milliwatts. Also, a high output power of several watts can be generated while low enough intensities ensure that thermal damage to any of the facets is avoided.

\subsubsection{Tapered amplifier setup}

The tapered amplifier used for modulation is fabricated by Eagleyard (EYP-TPA0780-01000-3006-CMT03-0000) and is based on a gallium arsenide semiconductor. It has an input facet width of $w_{1}=3 \mu \mathrm{m}$, an output facet width of $w_{2}=190 \mu \mathrm{m}$ and a total length of the active area of $L=2750 \mu \mathrm{m}$ [125] (compare Fig. 4.1). The output power is $1 \mathrm{~W}$ and it accepts an input power between $10 \mathrm{~mW}$ and $50 \mathrm{~mW}$, resulting in a typical gain of $13 \mathrm{~dB}$ to $20 \mathrm{~dB}$. The supply current can be up to $3 \mathrm{~A}$ and is provided here by a Thorlabs current controller (LDC240C) with a maximum output current of $\pm 4 \mathrm{~A}$.

The standard assembly of the tapered amplifier is described in the following. The tapered amplifier is mounted in a custom-built housing out of solid aluminum (see App. C for schematics). The housing (Fig. C.2) is completed with commercially available windows by Thorlabs (WG10530-B, N-BK7) with an anti-reflection coating from $650 \mathrm{~nm}$ to $1050 \mathrm{~nm}$ placed in a window holder (Fig. C.3) from the 
outside. This protects the tapered amplifier from dust and helps to stabilize the temperature inside the housing. The tapered amplifier chip itself is mounted on a solid copper mount (Fig. C.1) for efficient heat dissipation.

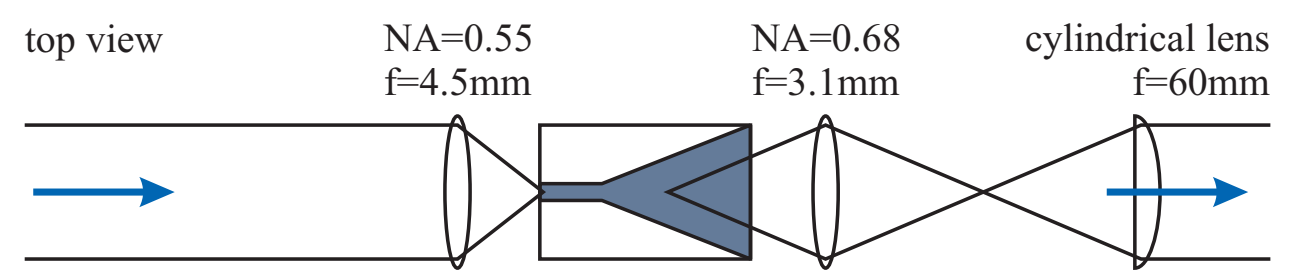

side view

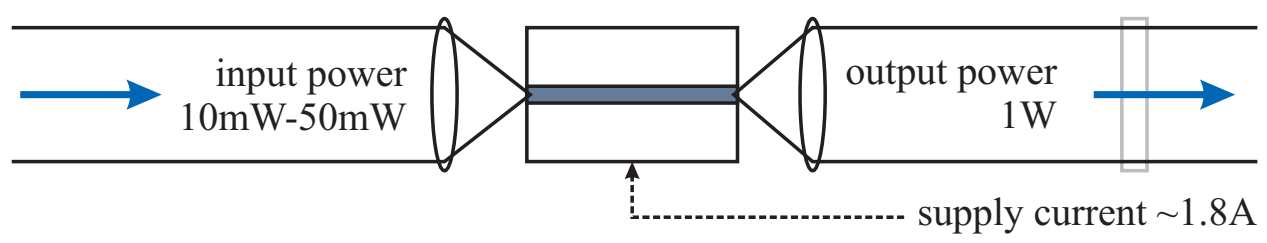

Figure 4.2.: Lens system for tapered amplifier. Top view and side view. The beam diverges horizontally due to the tapered shape. This has to be corrected by a cylindrical lens as seen in top view. The two lenses around the tapered amplifier chip are mounted in the housing, the cylindrical lens is mounted on a post outside. The drawing is based on Ref. [126].

Two small lenses can be positioned on the mount as well. They sit on two short metal rods each for stability and are glued with two-component epoxy. One lens from Thorlabs (C230TM-B) with a focal length of $4.5 \mathrm{~mm}$ is mounted in front of the tapered amplifier to focus the light into the active area. The other lens,also from Thorlabs (C330TM-B) with a focal length of $3.1 \mathrm{~mm}$, is mounted in the back to collimate the amplified beam. Due to the tapered shape of the amplifier, the divergence of the beam has to be corrected in the horizontal plane with an additional cylindrical lens with a focal length of $60 \mathrm{~mm}$ outside of the housing. The entire lens system is sketched in Fig. 4.2. The copper holder with the mounted tapered amplifier and the two lenses sit on a Peltier element to transport the heat to the housing. A thermistor at the copper mount measures the temperature close to the chip and a custom-built PID controller stabilizes the temperature to $20^{\circ} \mathrm{C}$. The electrical connection of the tapered amplifier is made by screwing one lead to the copper piece connected with the anode of the amplifier and soldering the cathode directly. Both lead wires are connected at the housing with banana jacks. The finished assembly can be seen from the top in Fig. 4.3a. 
a)

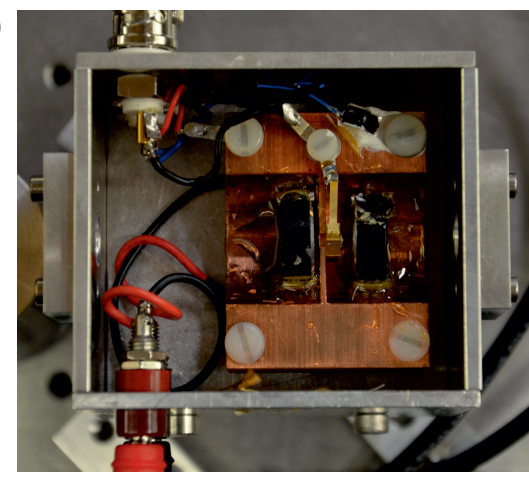

b)

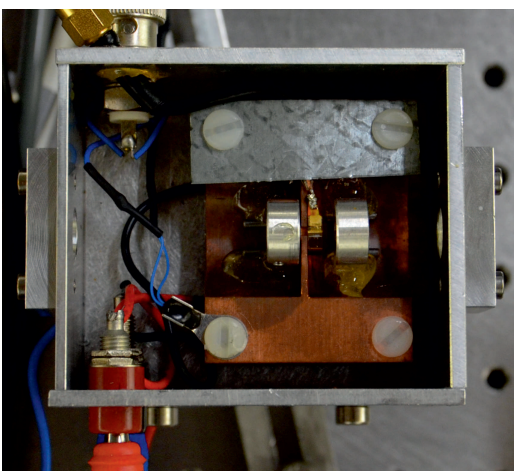

Figure 4.3.: Assembled tapered amplifier in standard and modulation setup. a) Standard assembly. The figure shows the tapered amplifier chip on the copper mount, which also holds the collimation lenses. Both electrical leads can be seen at the upper part of the copper mount, as well as the thermistor at the top right. The Peltier element is located below the mount. b) Modified assembly for the modulation of the supply current. The anode is now connected by pushing the outer lead of a stripped SubMiniature version A (SMA) cable to the mount with a metal sheet. The connector of the SMA cable can be seen just outside the housing. The thermistor moved to the lower left.

\subsubsection{Microwave source for the modulation of the supply current}

This section describes the microwave source for the generation of sidebands, as needed for direct modulation of a tapered amplifier. All frequencies are given for rubidium 87, as it is the atomic species used in the experiment. The frequency needed for modulation is the difference between the cooling and repumping light. The desired modulation frequency needs to be known beforehand to set up the microwave source. The cooling light is defined by the transition frequency $|F=2\rangle \rightarrow\left|F^{\prime}=3\right\rangle$ and the repumping light has the transition frequency $|F=1\rangle \rightarrow\left|F^{\prime}=2\right\rangle$ (see Fig. B.1). The difference between the two transitions [114] is the desired beat frequency needed for modulation:

$$
\Delta \omega=\omega_{F=1 \rightarrow F=2}-\omega_{F^{\prime}=2 \rightarrow F^{\prime}=3}=6834.7 \mathrm{MHz}-266.7 \mathrm{MHz}=6567.5 \mathrm{MHz} .
$$

Depending on the experimental step, a detuning of up to $28 \mathrm{MHz}$ is added, leading to a desired modulation frequency of $6596 \mathrm{MHz}$. This frequency in the microwave regime has to be generated by a suitable source (see Fig. 4.4). Here, a signal synthesizer from Marconi generates a seed signal at $132 \mathrm{MHz}$. This initial 


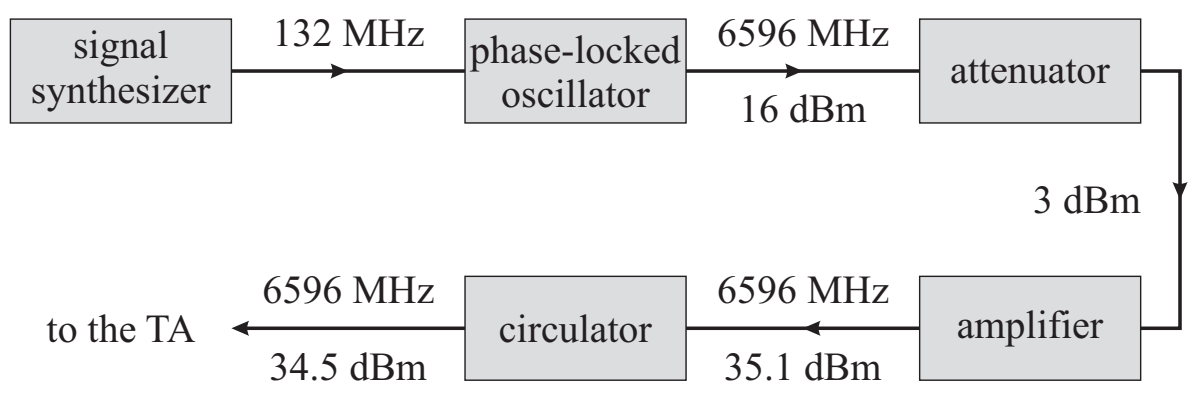

Figure 4.4.: Microwave supply chain. A synthesizer signal at $132 \mathrm{MHz}$ seeds an oscillator which multiplies the frequency by a factor of 50 to $6596 \mathrm{MHz}$. A microwave signal with a power of $34.5 \mathrm{dBm}$ is send to the tapered amplifier after an attenuator-amplifier combination and a circulator.

signal is multiplied by a factor of 50 with a phase-locked oscillator (Miteq BCO130-140-06750-15P) to reach the desired frequency of $6596 \mathrm{MHz}$. The oscillator has an output power of $16 \mathrm{dBm}$. A microwave amplifier (Minicircuits ZVE-3W$83+$ ) enhances the signal to $35.1 \mathrm{dBm}$. A preceding attenuator reduces the signal power by $13 \mathrm{~dB}$ to match the power level to the input specifications of the amplifier. Finally, a circulator (Microwave Technology Corporation H119VFF) with a $50 \Omega$-terminator prevents back reflections and thus damage to the amplifier. The circulator has an insertion loss of $0.6 \mathrm{~dB}$. This microwave source provides the desired microwave output which will be used to modulate the current of the tapered amplifier.

\subsubsection{Modulation setup}

It is essential to couple as much of the provided microwave power as possible into the chip of the tapered amplifier. Additionally, as in unmodulated operation, a constant current has to be applied. The tapered amplifier chip has to be supplied with a current of $1.8 \mathrm{~A}$ to amplify the typical seed with a power of $18 \mathrm{~mW}$ to $1 \mathrm{~W}$. This current is provided by a current driver (Thorlabs LDC240C). Both the microwave signal and the direct current can be combined and coupled into the tapered amplifier with a bias tee (see Fig. 4.5). Simplified, a bias tee is a three-way connector with a capacitor at the microwave input and a coil at the direct current input. The capacitor acts as a high pass filter, which is only transmissive for higher frequencies blocking the direct current part. This protects the microwave source from high currents at the direct current input. The coil at the direct current input is a low pass filter for the supply current. Due to its inductance, it blocks most of the microwave signal. The combination of the two signals is put out at the third port. Thus, the bias tee allows for the combination of high and low frequency 


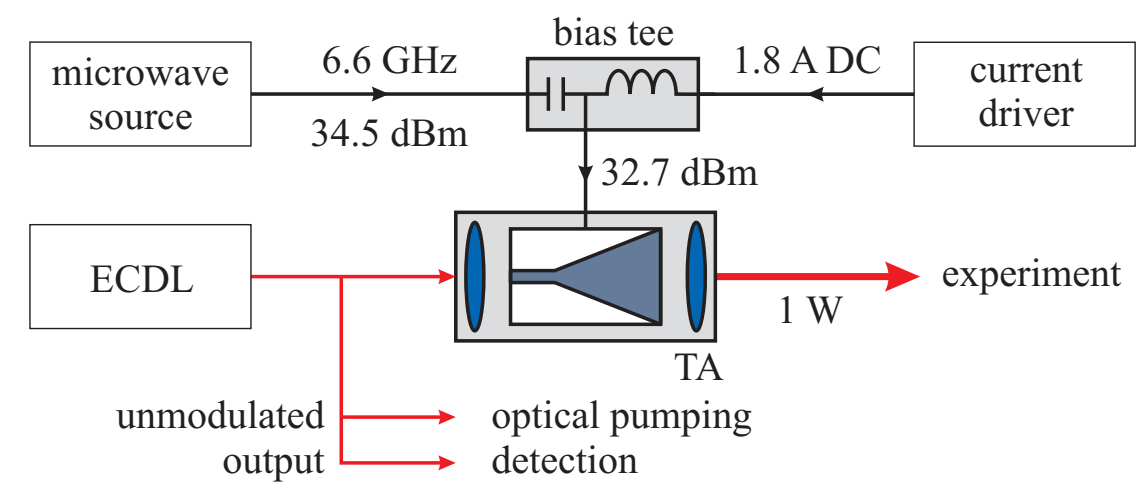

Figure 4.5.: Tapered amplifier modulation setup. The microwave and direct current signal are combined in a bias tee and used to power the tapered amplifier. An external-cavity diode laser delivers the seed signal. The sidebands for repumping are generated in the tapered amplifier, additionally the light is amplified to $1 \mathrm{~W}$. It is send to the experiment chamber with a fiber.

signals without crosstalk between the inputs.

The bias tee (Pasternack PE1615) is especially well suited to operate with high input currents up to $2.5 \mathrm{~A}$. The operation frequency is specified in the datasheet from $10 \mathrm{MHz}$ to $6000 \mathrm{MHz}$. Upon inquiry, the manufacturer assured compliance with the specifications for frequencies up to $7000 \mathrm{MHz}$. The bias tee is specified with an input loss of $1.5 \mathrm{~dB}$ at $7000 \mathrm{MHz}$, verified by a measurement. The power of the microwave signal behind the bias tee is thus $32.7 \mathrm{dBm}$.

The signal is applied to the tapered amplifier chip by soldering the inner lead of an SMA cable directly to the cathode of the chip, as described in the standard setup above. The anode of the chip is manufactured with an electrical connection on its own heat spreader and is connected to the copper mount by a metal screw. The connection from the SMA cable to the mount is made by pressing the stripped outer lead to the mount with a metal sheet (see Fig. 4.3b). It is important to have the SMA cable as short as possible to minimize emissions and losses. Therefore, the cable is connected to the bias tee just outside of the housing. A rudimentary impedance matching was performed by shortening the cable in very small steps and minimizing the reflections in the process. Still, almost all of the power is reflected. The implementation and characterization of this setup for modulation is described below. 
a)

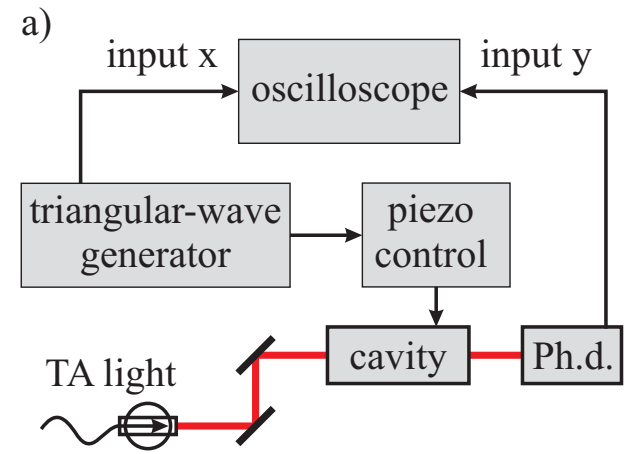

b)

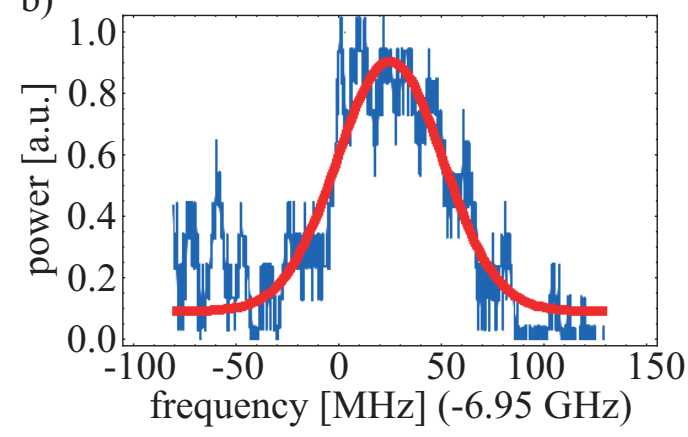

Figure 4.6.: Investigation of sideband creation with an external cavity. a) Cavity setup. A control signal from a triangular-wave generator has to be amplified to $80 \mathrm{~V}$ with a piezo control to drive the piezo mirror of the cavity. The cavity length change results in a voltage dependence of the light frequency transmitted to the photodiode. The photodiode signal combined with the triangular-wave signal are displayed at an oscilloscope in x-y-mode. b) Measurement signal comparison. The difference of the measurement signals from the setup to the left with and without modulation of the tapered amplifier current are plotted (blue) and fitted (red). The difference signal is proportional to the generated sidebands and shows the successful operation.

\subsection{Implementation in an experimental environment}

Now, the modulated supply current is applied to the tapered amplifier to generate the desired sidebands. To test the sideband generation in general, a simple cavity setup [121] is used (see Fig. 4.6a). For this first test, a non-stabilized diode laser is used to seed the tapered amplifier. The amplified and modulated light is coupled into a cavity. The cavity output is measured by a fast photodiode (Hamamatsu G4176-03). The length of the cavity selects which frequency is transmitted and is adjustable with a piezo actuator. By a modulation of the piezo actuator current, the transmitted frequencies can be analyzed. A clear distinction between the signal with and without microwave modulation (see Fig. 4.6b) can be seen. This is the first evidence for the successful generation of sidebands. 
After this first demonstration, a further investigation in the usability of the setup is performed in the experimental environment described in this thesis in Chapter 2. The goal is to operate a three-dimensional magneto-optical trap (3D-MOT) with all repumping light generated by the modulated tapered amplifier. The presently used additional diode laser for the repumping frequency must not be used for the three-dimensional trap, but is still used to operate the two-dimensional magnetooptical trap (2D-MOT). The mirror-MOT (see Chap. 2.8) consists of one horizontal retro-reflected beam, which is always without repumping light. This beam can stay unchanged in the modulation setup. The two $45^{\circ}$-beams however contain repumping light and are replaced by the modulation scheme. The two beams share a common fiber and are split behind it with a polarizing beam splitter. It is thus convenient to insert the modulated tapered amplifier in front of the fiber leading to the 3D-MOT.

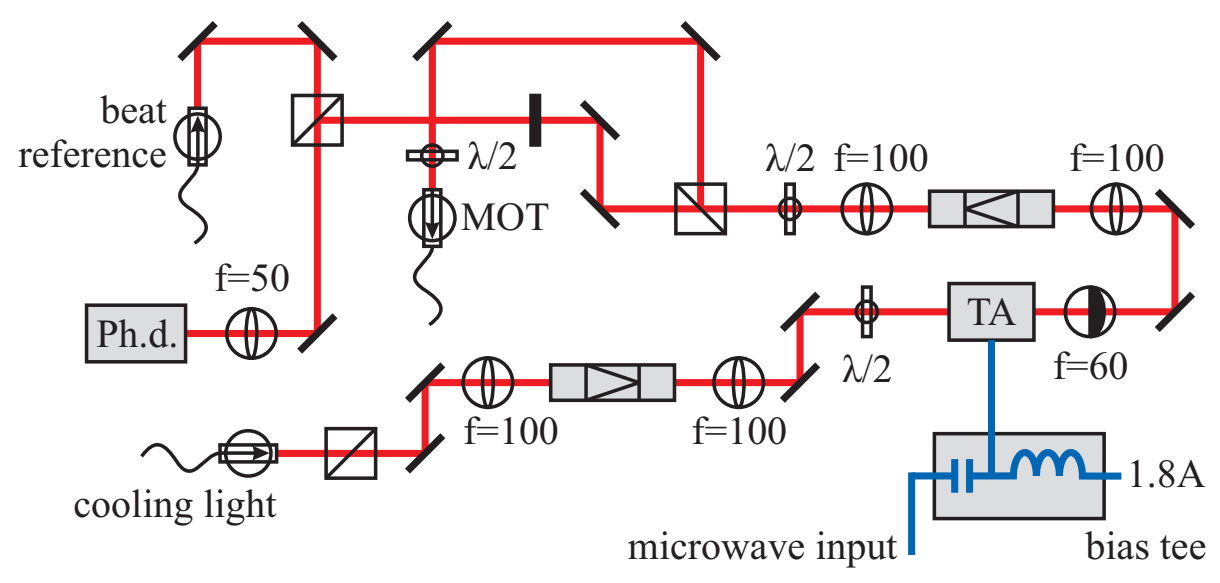

TA tapered amplifier $\odot$ fiber coupler (1) lens

$\square$ optical isolator

Ph.d. photodiode polarizing beam splitter

mirror, attenuator
(1) cylindrical lens

$\$ \lambda / 2$-wave plate

Figure 4.7.: Implementation of the modulated tapered amplifier into the experimental environment. The system to replace the conventional repumping light for the 3D-MOT is shown. Sidebands suitable for repumping are added in the tapered amplifier through modulation of the cooling light without repumping light. The modified light is send to the 3D-MOT in a fiber. A beat measurement is used to evaluate the sideband generation.

The entire modulation setup is compact and easily fits onto a small and movable, 
additional optics table. The optics part of the setup is drawn in Fig. 4.7. The previously used cooling light for the $45^{\circ}$-beams is led to the side table in an optical fiber. This light is usually only cooling light without repumping light, but if desired, can contain repumping light for comparison later on. A polarizing beam splitter is used to ensure a well defined polarization and an optical isolator (Isowave I-80-U2) eliminates back-reflections. A telescope out of two lenses with a focal length $f=100$ around the isolator helps to focus through the small aperture of the isolator and limit the losses to $20 \%$. Tapered amplifiers are sensitive to the polarization of the seed and a $\lambda / 2$-wave plate is used to adjust it. The tapered amplifier is seeded with the light and supplied by the modulated current described earlier. A cylindrical lens collimates the divergent beam from the tapered amplifier. Behind it, an optical isolator (Isowave I80-T4-M) prevents damage to the tapered amplifier from back-reflections. Again, a telescope minimizes losses. A polarizing beam splitter cube combined with a $\lambda / 2$-wave plate is used to adjust the power allocation of the light in two different beam paths. One is leading into the fiber for the $45^{\circ}$-MOT-beams. The other path is used to set up a beat measurement by overlapping additional light and shining the combination onto a high speed photodiode (Hamamatsu G4176-03).

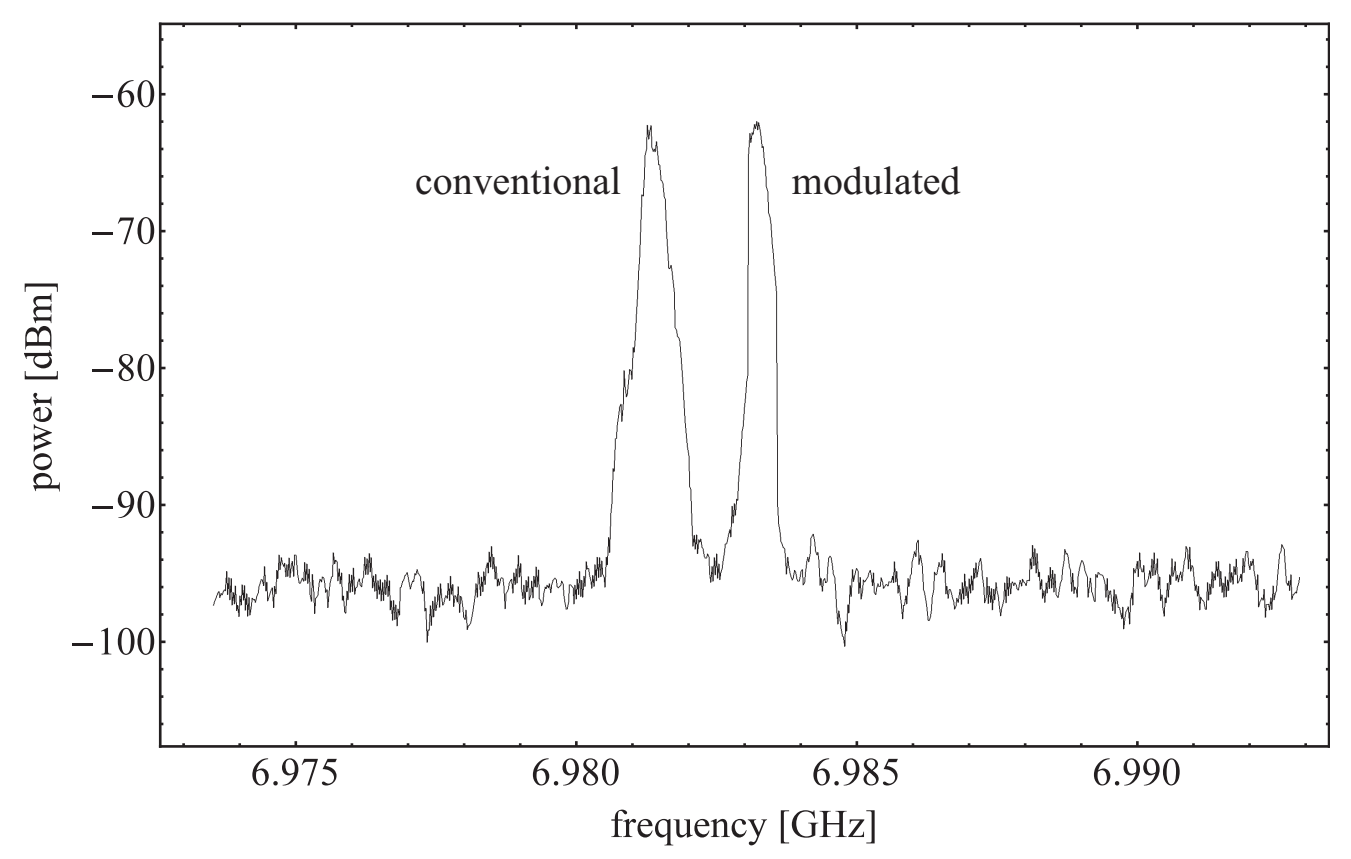

Figure 4.8.: Efficiency of the repumping sideband generation. The beat signal between a reference beam and the conventional but reduced repumping light can be seen on the left. On the right is the beat signal from the same reference laser with the repumping light created as sidebands through modulation of the tapered amplifier current. 
A beat measurement is a valuable tool to measure the relative power of different signals. To make this comparative measurement, two separate signals have to be investigated. As a reference, modulation is turned off and the tapered amplifier is only used to amplify the light. Instead of modulation, repumping light from the second diode laser is used to operate a 3D-MOT. Now, the power of the repumping light is reduced such that the operation of a MOT is still possible (more than $10^{8}$ atoms can be loaded). A fraction of this light is used in a beat measurement mentioned above. To create a beat signal, it is superimposed by cooling light shifted by additional $382 \mathrm{MHz}$. This shift is necessary because parasitic stray electric fields couple into the photodiode. The shift enables the distinction between the beat signal and erroneous signals due to stray fields. Due to this shift, the beat signal is now expected around $6596 \mathrm{MHz}+382 \mathrm{MHz}=6978 \mathrm{MHz}$ and can be seen in Fig. 4.8 as peak on the left. A signal of at least this strength has to be created by modulation to be able to operate a 3D-MOT.

The beat signal from modulation is created in a similar way. First of all, the conventional repumping light is blocked completely. Then, the modulation of the tapered amplifier is used as described above to generate the sidebands. Next, a beat signal is created between this light with the modulated sidebands and the shifted reference light. The beat is again detected with the photodiode and leads to the signal peak on the right in Fig. 4.8. Both beat measurements can be performed simultaneously to create the whole beat signal seen in the mentioned figure. A comparison of the magnitude of the two signals shows an equal power in both peaks. A further comparison of the carrier power and the sideband power yields a rough estimate of the relative power $P_{\text {sidebands }} / P_{\text {carrier }}=0.003$ in each sideband. Hence, the operation of the 3D-MOT with repumping sidebands should be feasible.

\subsection{Characterization of the sidebands using a magneto-optical trap}

The modulation setup is implemented in the experimental apparatus and the operation of a MOT is evaluated. For this purpose, the 3D-MOT light contains repumping light from modulation only, while the conventional repumping light is still used for the 2D-MOT and detection for better comparability. The time dependence of the MOT loading is plotted in Fig. 4.9a. A fit to the experimental data shows that the initial loading rate is $1.6 \times 10^{9}$ atoms $/ \mathrm{s}$ reaching a steady-state number of atoms of $1.1 \times 10^{9}$ after $1.7 \mathrm{~s}$.

The MOT performance is limited by the power of the microwave actually reaching the tapered amplifier chip. The number of atoms in the fully loaded 3D-MOT dependent on the microwave power behind the bias tee is depicted in Fig. 4.9b. The power is reduced step-by-step with a combination of different passive atten- 
a)

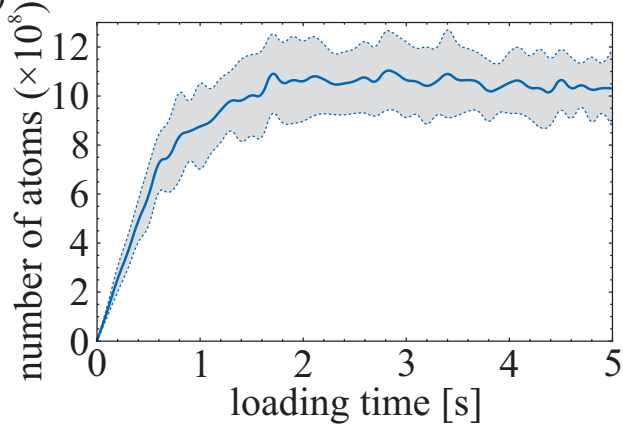

b)

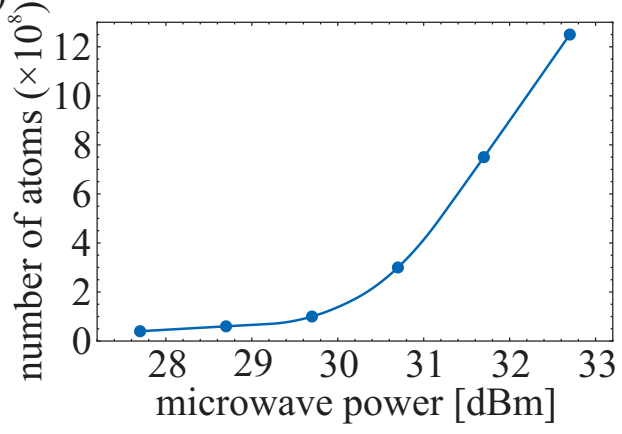

Figure 4.9.: Number of atoms in the 3D-MOT. a) Loading time. Number of atoms in the 3D-MOT as a function of the loading time. The shaded area corresponds to the standard deviation of 6 images. b) Microwave power. Number of atoms in the fully loaded 3D-MOT depending on the microwave power behind the bias tee. The solid line is a guide to the eye.

uators. The graph shows a strong correlation and no saturation yet, implying a further increase in the number of atoms with an increased microwave power. The temperature of the MOT was also evaluated with a series of time-of-flight images to be $121 \mu \mathrm{K}$. A $\sigma^{+}-\sigma^{-}$-optical molasses [127] with a duration of $1.3 \mathrm{~ms}$ could reduce the temperature to $87 \mu \mathrm{K}$, which compares well to the results of the conventional repumper.

The power of the sidebands also influences the lifetime in the 3D-MOT. A lower power would let the atoms stay longer in the $|F=1\rangle$-state, so that they can leave the trap before being brought back into the cooling cycle. This would reduce the lifetime in the trap. The actual lifetime was determined by loading the 3D-MOT and then switching off the 2D-MOT while monitoring the decreasing number of atoms as a function of the holding time (Fig. 4.10a). The resulting $1 / e$-lifetime of $0.84 \mathrm{~s}$ is calculated from a fit.

As a final measurement, the frequency dependence is investigated by tuning the initial modulation frequency at the signal synthesizer. The corresponding change in the number of atoms is detected and plotted in Fig. 4.10b. Apparently, the number of atoms reaches its maximum, when the repumping light is in resonance with the repumping transition $|F=1\rangle \rightarrow\left|F^{\prime}=2\right\rangle$. The transition is asymmetric, declining slower with a red-detuned microwave, because this also realizes the MOTconfiguration for the repumping states. 
a)

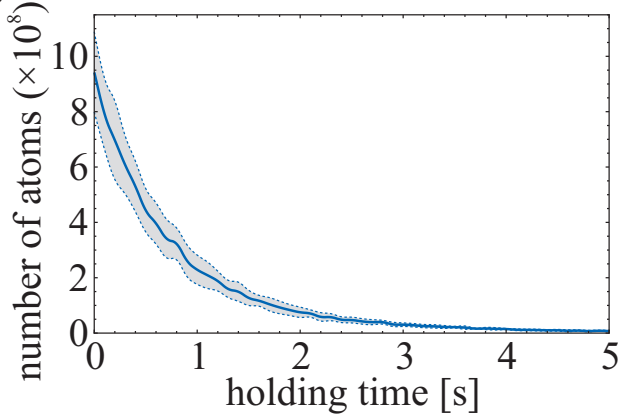

b)

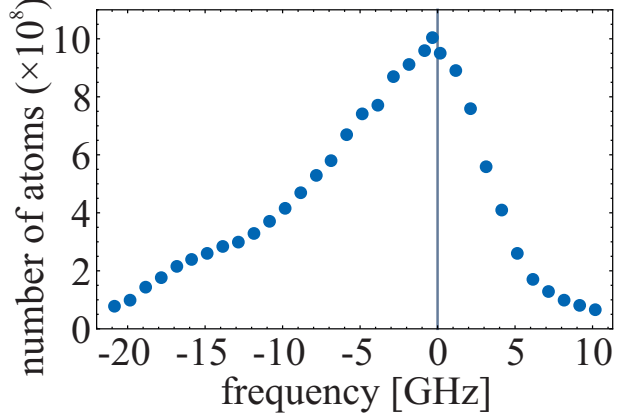

Figure 4.10.: 3D-MOT lifetime and frequency dependence. a) Lifetime. Number of atoms in the 3D-MOT as a function of the holding time after the 2D-MOT is switched off. The loading time is $1 \mathrm{~s}$. The shaded area corresponds to the standard deviation of 6 images. b) Frequency dependence. Number of atoms in the 3D-MOT as a function of the optical sideband detuning with respect to the repumping transition. The solid line indicates the resonance frequency of the $|F=1\rangle \rightarrow\left|F^{\prime}=2\right\rangle$ transition.

\subsection{Conclusion}

The tapered amplifier modulation presented in this chapter can provide repumping and cooling light for the operation of a 3D-MOT with $1.1 \times 10^{9}$ atoms. The number of trappable atoms is limited by the power in the sidebands and thus by the microwave power coupled into the tapered amplifier chip. This coupling efficiency could be increased by a more sophisticated impedance matching or a better suited tapered amplifier model. Even though, the performance is a factor of ten less than the best performance of the apparatus, there are many advantages to the modulation of the tapered amplifier. The setup is compact and simple, making it an ideal modification for mobile or space applications. Additionally, it is very versatile and flexible, as it can produce several frequencies while still keeping the initial seed without modulation for manipulation and detection. The modulation frequency can be adjusted during operation and is tunable [128] over a wide range of up to $7 \mathrm{GHz}$. The carrier and sidebands are emitted from the same source locking their mode, phase and polarization. This enables further applications, including Raman spectroscopy or the cooling of molecules. 



\section{Outlook}

A continuous reservoir of ultracold atoms can benefit many fields of research including sympathetic cooling of atoms, molecules and nanoscopic objects. Such a continuously refilled reservoir in the millikelvin regime was presented for the first time in this thesis. The reservoir is achieved by accumulating atoms from a continuous beam in a static magnetic trap. The trap contains $3.8 \times 10^{7}$ atoms in the steady state at a temperature of $102 \mu \mathrm{K}$. The loading scheme is implemented on a versatile mesoscopic atom chip. The accumulation does not rely on any specific atomic properties and can thus be adopted to any other trappable particle. Currently, this scheme produces the coldest continuous reservoir maintained without laser light.

The proposal [30] suggests, that reaching quantum degeneracy in the continuous reservoir is possible. In the following, several ways to get closer to this goal are presented. One option is to increase the phase-space density in the trap to improve the continuous atom beam loading the trap. Its temperature and flux are currently limited by residual magnetic fields during the molasses phase. These residual fields arise from eddy currents in the copper mount of the mesoscopic atom chip. Even though the currents producing the magnetic fields can be switched off quickly, the magnetic fields still persist for about $20 \mathrm{~ms}$. To avoid eddy currents in the future, an updated version of the mesoscopic atom chip is currently implemented (see Fig. 5.1). A $1 \mathrm{~cm}$ slice of the copper base at the bottom of the atom chip is replaced by the ceramic Shapal, a machinable aluminium nitride ceramic with a large heat conductivity. A test measurement showed a strong suppression of eddy currents with this design. Calculations showed that the molasses phase without eddy currents can improve the transfer efficiency to the guide by a factor of 15 . For this case, the molecular dynamics simulation predicts an increase of the phasespace density by a factor of 80 (see Fig. 5.2).

The atom chip is further enhanced by the update. The connection of the chip wires to the supply lines are strengthened. These connections were the bottleneck in the current chip design, heating up most under constant high currents. The new connectors will lift this restriction and allow for constant currents up to $\pm 320 \mathrm{~A}$ which is the full potential of the current stabilization. The increased currents will improve both the trap depth as well as the trapping frequencies.

Additional perpendicular wires on the atom chip will be used to enhance the trapping potential. The proposal [30] predicts favorable collisional conditions for strongly elongated traps. In the current setup, a ratio of the radial to the lon- 


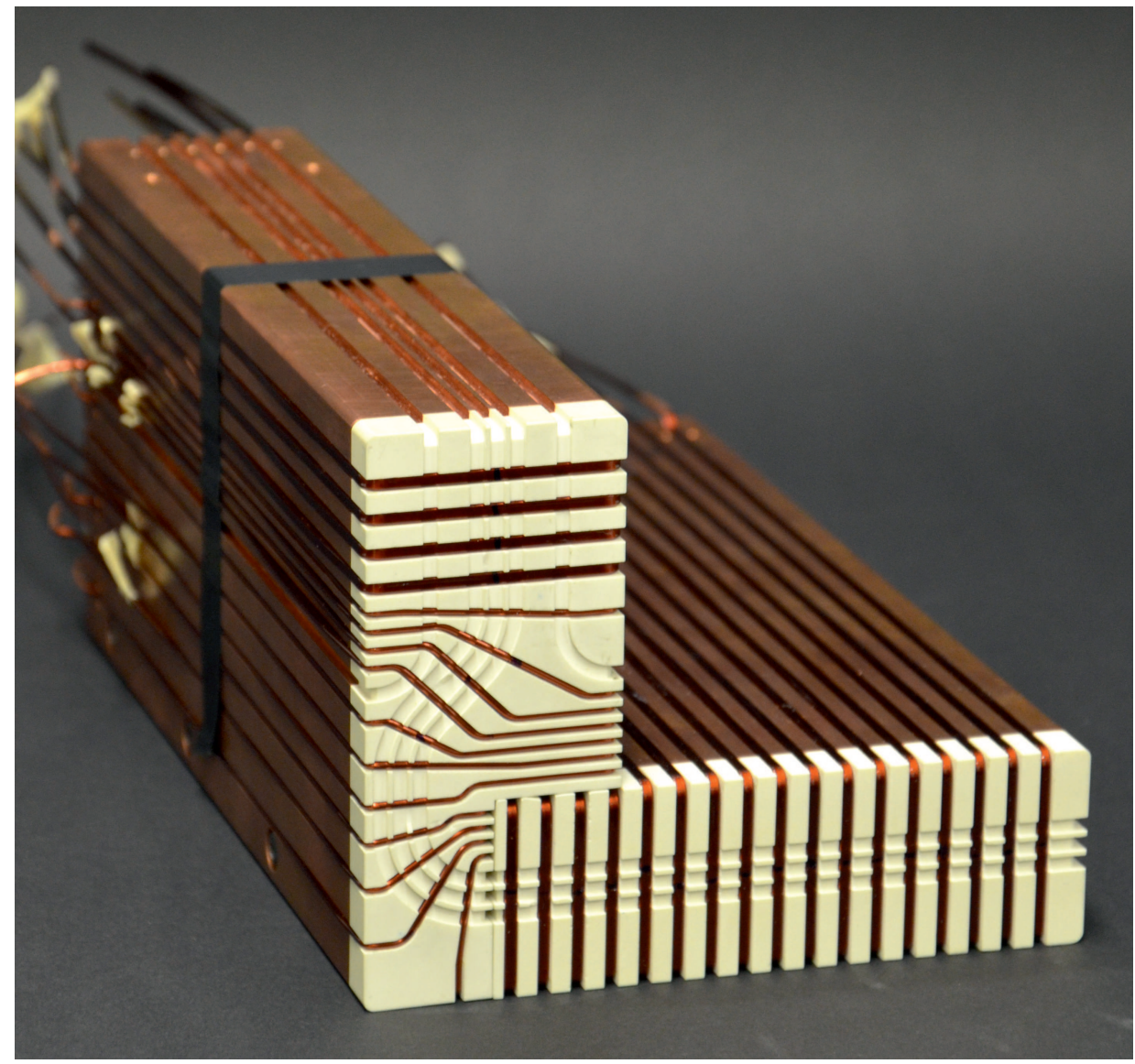

Figure 5.1.: Next generation of the mesoscopic atom chip. Improvements include a ceramic bottom facet to reduce eddy currents, additional perpendicular wires and a better wire connection to the supply lines. The photo shows the atom chip without guide wires and connectors.

gitudinal trap frequencies of 30 is achieved. The use of additional perpendicular wires can elongate the trap further and drastically increase this ratio. Replacing the current optical dipole trap by a model with a power of $100 \mathrm{~W}$ at a wavelength of $1064 \mathrm{~nm}$ can strongly increase the trap frequencies and increase the trap depth from $750 \mu \mathrm{K}$ to $3400 \mu \mathrm{K}$. This trap depth is enough to investigate a resonance in the transverse evaporation threshold lying currently above our trap depth. It is predicted that bringing the evaporation threshold to the resonance will increase the phase space density by a factor of 500 [30]. The combination of these improvements will push the scheme towards the continuous operation of a quantum degenerate sample.

With the new atom chip, another approach towards a continuous quantum degenerate cloud is possible. The added perpendicular wires allow for a controlled 


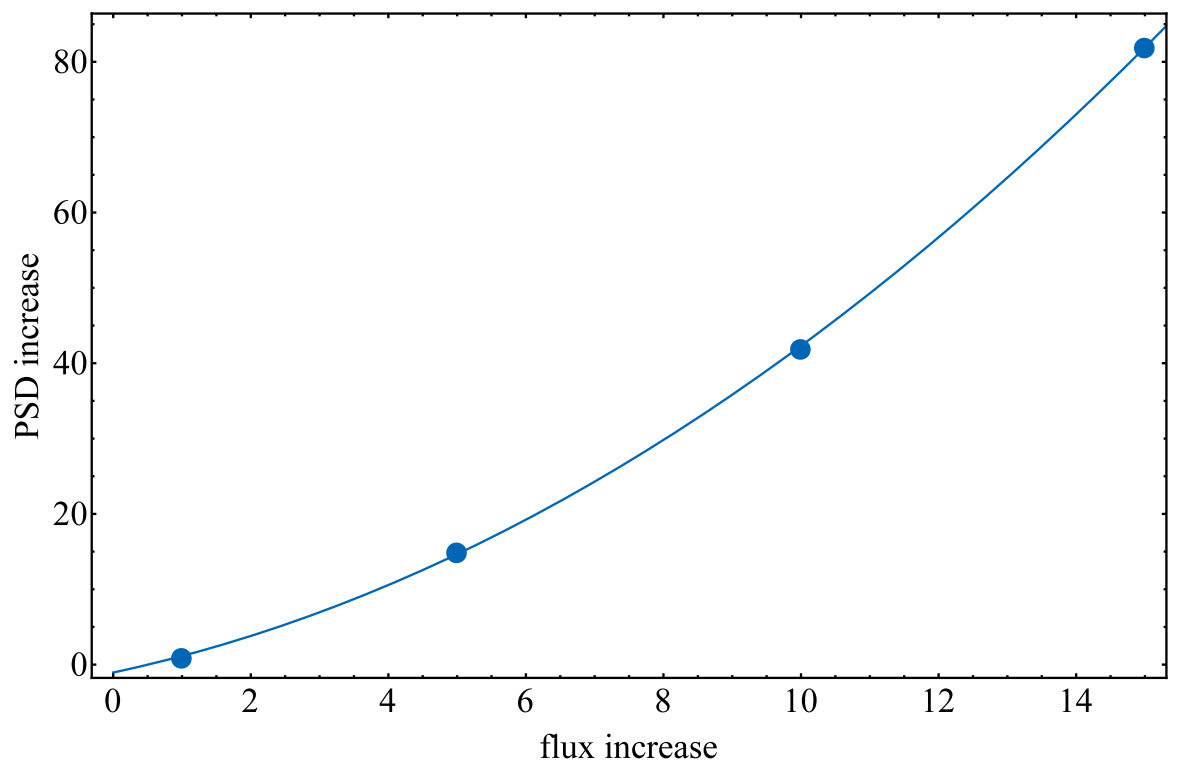

Figure 5.2.: Phase-space density change in the reservoir depending on the change of flux of the incoming beam. The datapoints are a prediction of the phase-space density change by a molecular dynamics simulation.

sequential transport of atomic clouds towards the trapping area. When evaporatively cooled along the way, the clouds can be used to sustain a Bose-Einstein condensate at the end of the guide. The production of a single Bose-Einstein condensate in the retrapping are has been demonstrated previously [102] and was achieved by evaporative cooling with a microwave. This approach is not feasible with several trap sites close to each other, as the microwave would address all equally. Spatial evaporation can be used as a good alternative. For this technique, a surface is brought close to the trap site. The hotter atoms in the trap tend to have a larger distance to the trap center and can thus collide with the surface. It has been shown, that this method is as effective as microwave evaporation [129] and can also be applied to atomic beams [130]. The clouds that are thus cooled during the transport can then be merged at the end of the guide. Such a merging of Bose-Einstein condensates has been demonstrated previously in an optical dipole trap [131]. It is also possible to extract a matter beam from a magnetic trap while replenishing the losses by another [132], thus creating a quasi-continuous atom laser.

The continuously replenished reservoir of ultracold atoms presented in this thesis is a promising tool for the sympathetic cooling of atoms, molecules and nanoscopic objects and as a resource for metrology. When reaching quantum degeneracy, a continuously replenishes reservoir can be used to create the first continuous-wave atom laser [133]. 



\section{A. Vacuum components}

Here, the assembled vacuum chamber is depicted and the mounting of the mesoscopic atom chip in the flange with the L-shaped recess is illustrated.

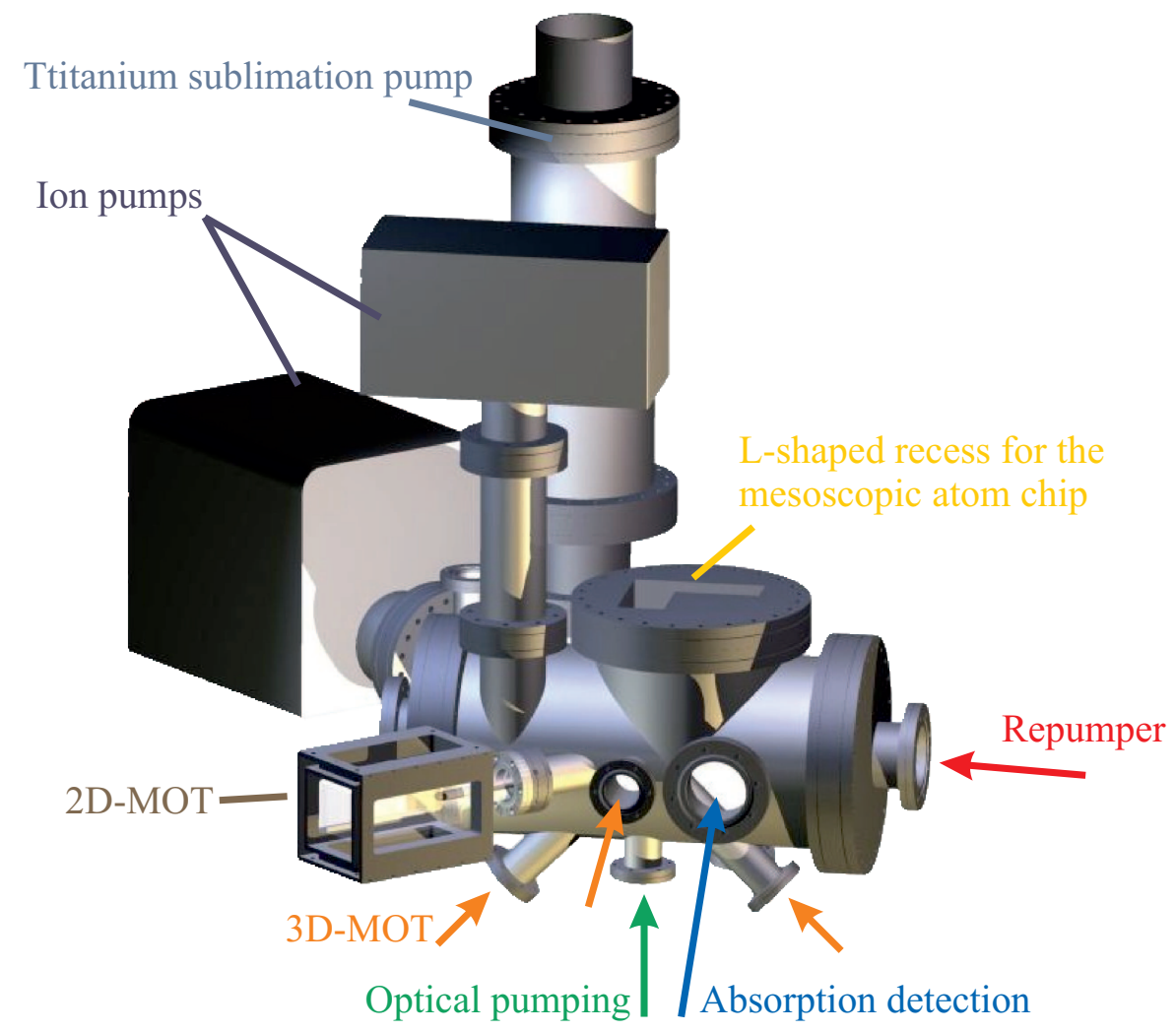

Figure A.1.: The vacuum chamber. Visible are the vacuum pumps, the 2DMOT cell, the view ports for the 3D-MOT, optical pumping, repumper and detection mounted on the chamber. A flange with an L-shaped recess is located on top to house the atom chip. 


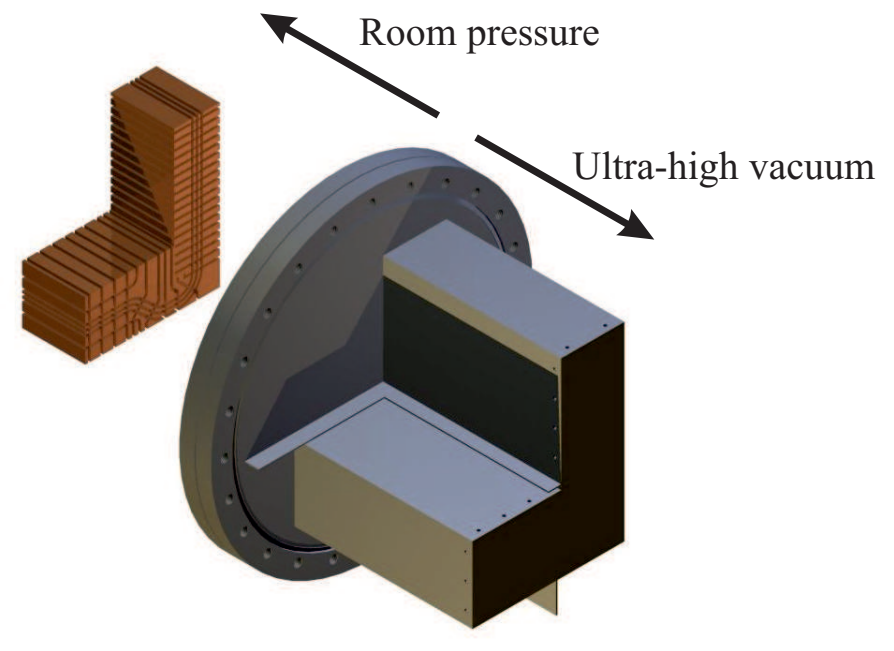

Figure A.2.: Flange with L-shaped recess. The mesoscopic atom chip is lowered into the flange from the outside of the vacuum chamber, thus the atom chip can be exchanges while the apparatus remains evacuated. 


\section{B. Rubidium $87 \mathrm{D}_{2}$ transition hyperfine structure}

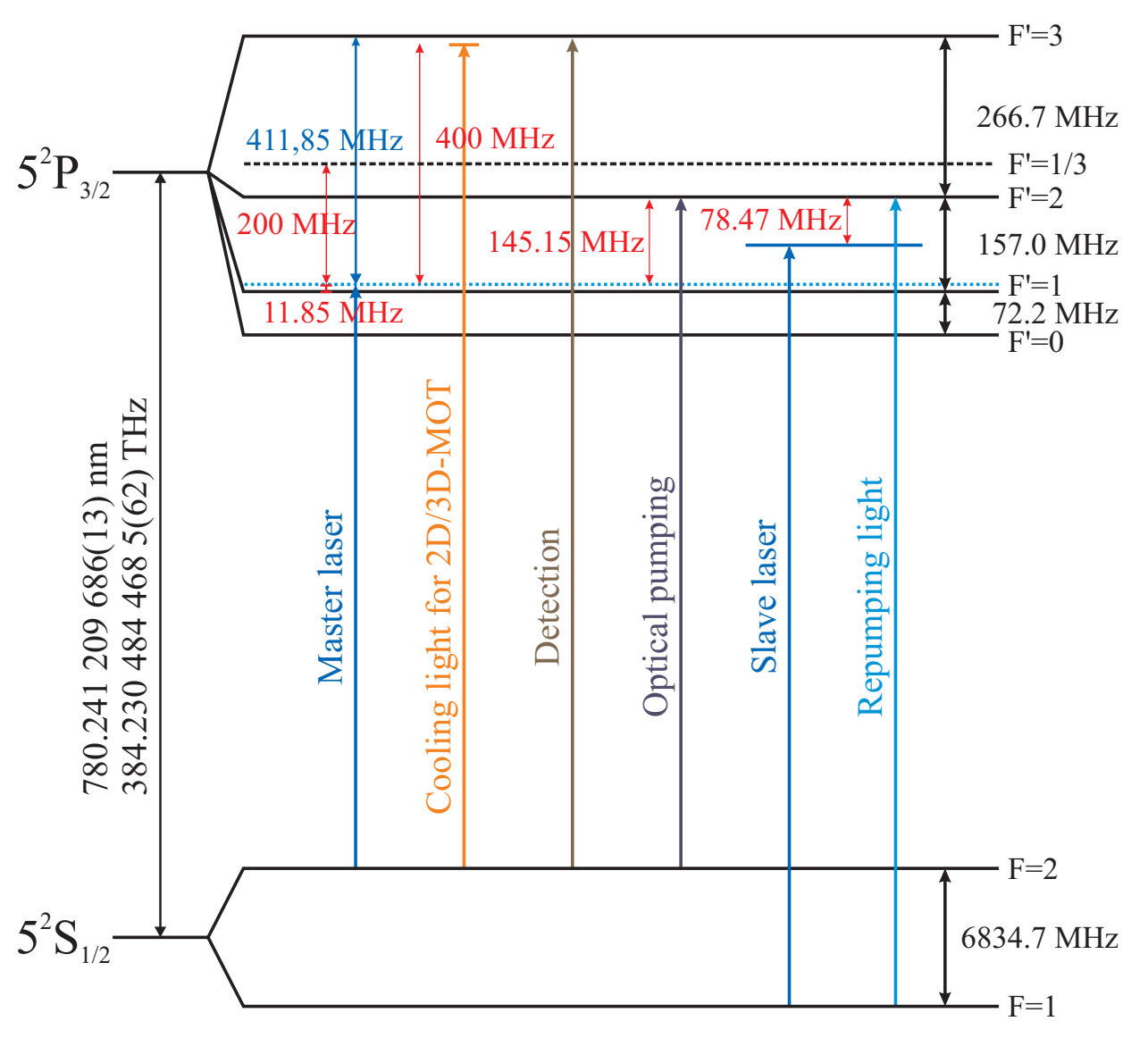

Figure B.1.: Rubidium $87 \mathrm{D}_{2}$ transition hyperfine structure. Hyperfine structure with the relevant optical transitions used in the experiment. The transitions from the $F=2$ state are derived from the master laser through accousto-optical modulation, and the repumping light is derived from the slave laser. The values are from Ref. [114]. 



\section{Tapered amplifier housing}

This section contains the technical drawing for the mechanical parts necessary to assemble a tapered amplifier housing.

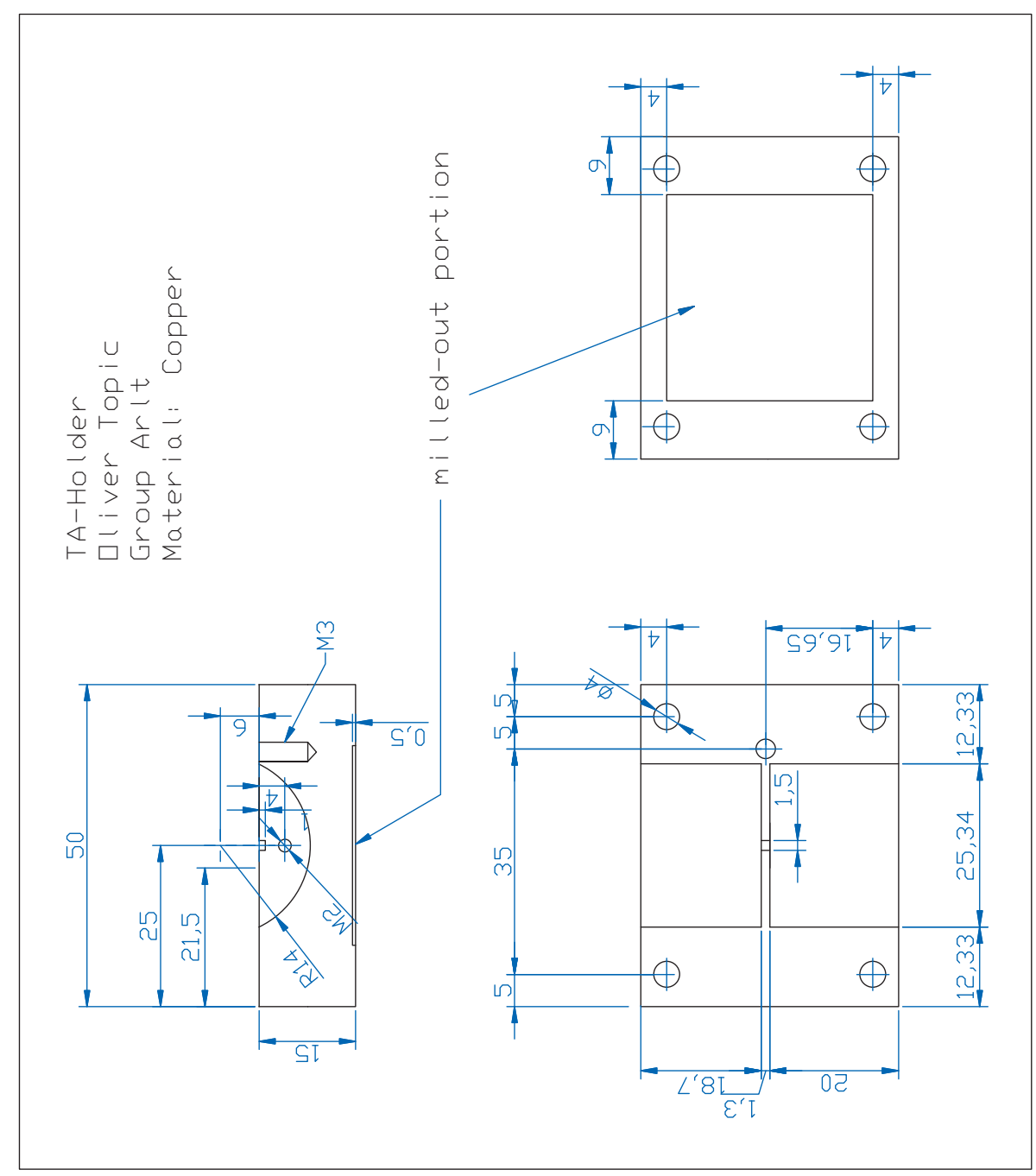

Figure C.1.: Tapered amplifier holder. The tapered amplifier is screwed onto the bridge. The two lenses are glued into the round hollow. Material: Copper. 


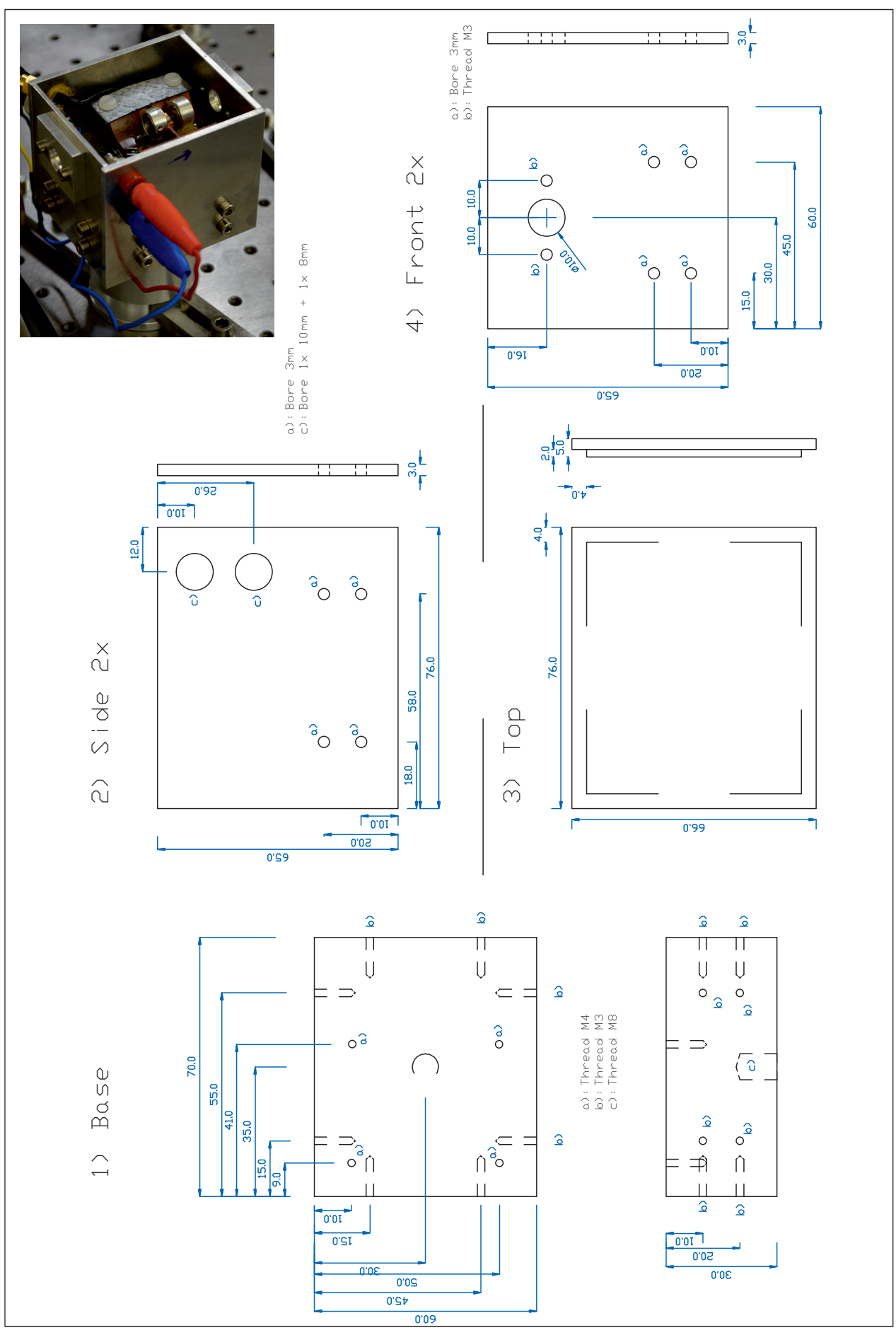

Figure C.2.: Tapered amplifier housing. The holder (Fig. C.1) is screwed on the base at the four points a). Material: Aluminum. The assembled housing is pictured in the inset. 
a): Bore $\varnothing$ 3.2 $\mathrm{mm}$

b): Bore $\varnothing 10.0 \mathrm{~mm}$

$+\varnothing$ 13.0 mm recess, 4.0 mm deep
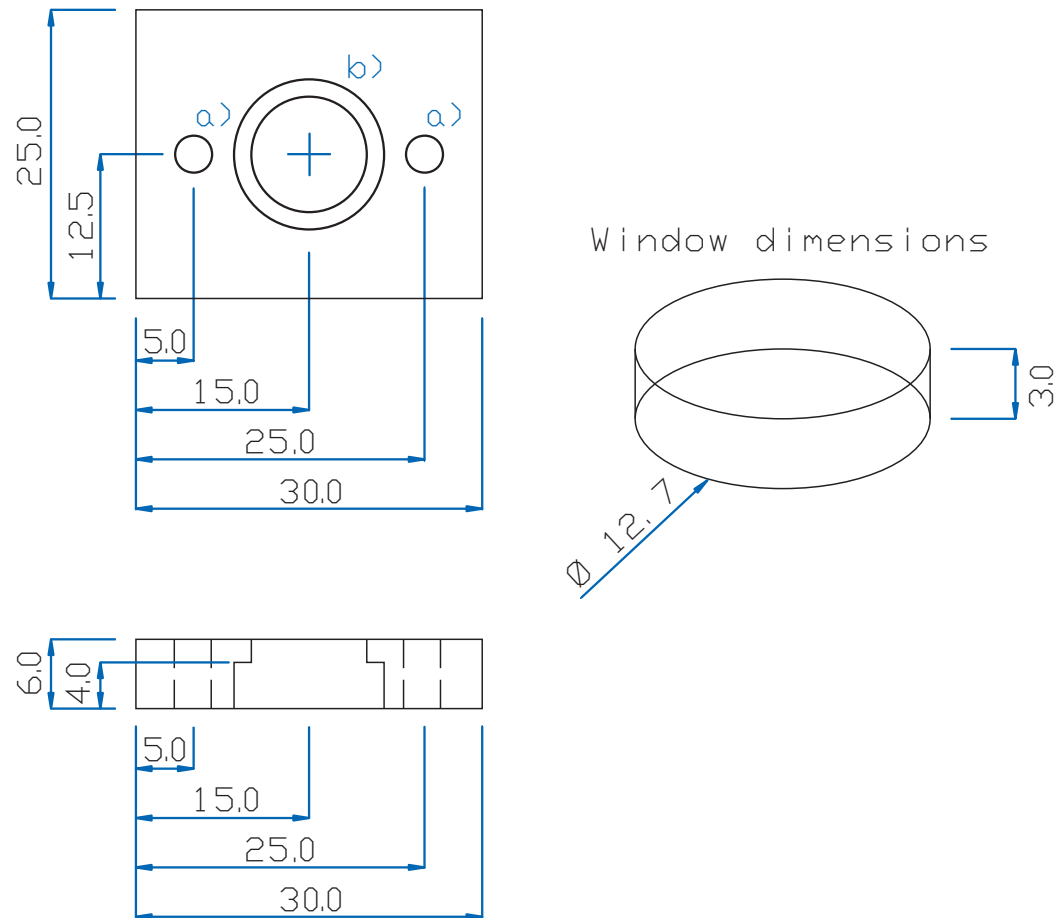

Figure C.3.: Tapered amplifier window holder. Material: Aluminum. The holder is screwed from the outside to the housing (Fig. C.2), enclosing the window in the recess inbetween. The window is manufactured by Thorlabs (WG10530-B, N-BK7) with an anti-reflection coating for $650 \mathrm{~nm}$ to $1050 \mathrm{~nm}$, a diameter of $12.7 \mathrm{~mm}$ and a thickness of $3 \pm 0.3 \mathrm{~mm}$. The recess in the holder is larger than the window thickness to eliminate strain on the window and avoid cracking. 



\section{Bibliography}

[1] W. D. Phillips and H. Metcalf. Laser deceleration of an atomic beam. Phys. Rev. Lett., 48, 596 (1982). (Cited on pages 1, 6, 8 and 9.)

[2] J. V. Prodan, W. D. Phillips, and H. Metcalf. Laser Production of a Very Slow Monoenergetic Atomic Beam. Phys. Rev. Lett., 49, 1149 (1982). (Cited on pages 1 and 6. )

[3] W. Ertmer, R. Blatt, J. L. Hall, and M. Zhu. Laser Manipulation of Atomic Beam Velocities: Demonstration of Stopped Atoms and Velocity Reversal. Phys. Rev. Lett., 54, 996 (1985). (Cited on pages 1 and 6.)

[4] S. Chu. Nobel Lecture: The manipulation of neutral particles. Rev. Mod. Phys., 70, 685 (1998). (Cited on pages 1 and 11.)

[5] C. N. Cohen-Tannoudji. Nobel Lecture: Manipulating atoms with photons. Rev. Mod. Phys., 70, 707 (1998). (Cited on pages 1 and 11.)

[6] W. D. Phillips. Nobel Lecture: Laser cooling and trapping of neutral atoms. Rev. Mod. Phys., 70, 721 (1998). (Cited on pages 1 and 11.)

[7] M. H. Anderson, J. R. Ensher, M. R. Matthews, C. E. Wieman, and E. A. Cornell. Observation of Bose-Einstein Condensation in a Dilute Atomic Vapor. Science, 269, 198 (1995). (Cited on pages 1, 7 and 27.)

[8] K. B. Davis, M. O. Mewes, M. R. Andrews, N. J. van Druten, D. S. Durfee, D. M. Kurn, and W. Ketterle. Bose-Einstein Condensation in a Gas of Sodium Atoms. Phys. Rev. Lett., 75, 3969 (1995). (Cited on pages 1, 7 and 27.)

[9] C. C. Bradley, C. A. Sackett, J. J. Tollett, and R. G. Hulet. Evidence of Bose-Einstein Condensation in an Atomic Gas with Attractive Interactions. Phys. Rev. Lett., 75, 1687 (1995). (Cited on pages 1, 7 and 27.)

[10] J. Rudolph, W. Herr, C. Grzeschik, T. Sternke, A. Grote, M. Popp, D. Becker, H. Müntinga, H. Ahlers, A. Peters, C. Lämmerzahl, K. Sengstock, N. Gaaloul, W. Ertmer, and E. M. Rasel. A high-flux BEC source for mobile atom interferometers. New J. Phys., 17, 065001 (2015). (Cited on page 1.) 
[11] T. Lévèque, A. Gauguet, F. Michaud, F. Pereira Dos Santos, and A. Landragin. Enhancing the Area of a Raman Atom Interferometer Using a Versatile Double-Diffraction Technique. Phys. Rev. Lett., 103, 080405 (2009). (Cited on page 1.)

[12] J. K. Stockton, K. Takase, and M. A. Kasevich. Absolute Geodetic Rotation Measurement Using Atom Interferometry. Phys. Rev. Lett., 107, 133001 (2011). (Cited on page 1.)

[13] G. Tackmann, P. Berg, C. Schubert, S. Abend, M. Gilowski, W. Ertmer, and E. M. Rasel. Self-alignment of a compact large-area atomic Sagnac interferometer. New J. Phys., 14, 015002 (2012). (Cited on page 1.)

[14] M. Kasevich and S. Chu. Atomic interferometry using stimulated Raman transitions. Phys. Rev. Lett., 67, 181 (1991). (Cited on page 1.)

[15] H. J. McGuinness, A. V. Rakholia, and G. W. Biedermann. High data-rate atom interferometer for measuring acceleration. Appl. Phys. Lett., 100, 011106 (2012). (Cited on page 1.)

[16] A. Louchet-Chauvet, S. Merlet, Q. Bodart, A. Landragin, F. Pereira Dos Santos, H. Baumann, G. D'Agostino, and C. Origlia. Comparison of 3 Absolute Gravimeters Based on Different Methods for the e-MASS Project. IEEE Trans. Instrum. Meas., 60, 2527 (2011). (Cited on page 1.)

[17] J. B. Fixler, G. T. Foster, J. M. McGuirk, and M. A. Kasevich. Atom Interferometer Measurement of the Newtonian Constant of Gravity. Science, 315, 74 (2007). (Cited on page 1.)

[18] G. Lamporesi, A. Bertoldi, L. Cacciapuoti, M. Prevedelli, and G. M. Tino. Determination of the Newtonian Gravitational Constant Using Atom Interferometry. Phys. Rev. Lett., 100, 050801 (2008). (Cited on page 1.)

[19] R. Wynands and S. Weyers. Atomic fountain clocks. Metrologia, 42, S64 (2005). (Cited on page 1.)

[20] C. Deutsch, F. Ramirez-Martinez, C. Lacroûte, F. Reinhard, T. Schneider, J. N. Fuchs, F. Piéchon, F. Laloë, J. Reichel, and P. Rosenbusch. Spin SelfRephasing and Very Long Coherence Times in a Trapped Atomic Ensemble. Phys. Rev. Lett., 105, 020401 (2010). (Cited on page 1.)

[21] G. Kleine Büning, J. Will, W. Ertmer, E. Rasel, J. Arlt, C. Klempt, F. Ramirez-Martinez, F. Piéchon, and P. Rosenbusch. Extended Coherence Time on the Clock Transition of Optically Trapped Rubidium. Phys. Rev. Lett., 106, 240801 (2011). (Cited on page 1.) 
[22] S. Dimopoulos, P. W. Graham, J. M. Hogan, M. A. Kasevich, and S. Rajendran. Atomic gravitational wave interferometric sensor. Phys. Rev. D, 78, 122002 (2008). (Cited on page 1.)

[23] P. Delva and E. Rasel. Matter wave interferometry and gravitational waves. J. Mod. Opt., 56, 1999 (2009). (Cited on page 1.)

[24] J. M. Hogan, D. M. S. Johnson, S. Dickerson, T. Kovachy, A. Sugarbaker, S.w. Chiow, P. W. Graham, M. A. Kasevich, B. Saif, S. Rajendran, P. Bouyer, B. D. Seery, L. Feinberg, and R. Keski-Kuha. An atomic gravitational wave interferometric sensor in low earth orbit (AGIS-LEO). Gen. Relativ. Gravit., 43, 1953 (2011). (Cited on page 1.)

[25] M. Hohensee, S.-Y. Lan, R. Houtz, C. Chan, B. Estey, G. Kim, P.-C. Kuan, and H. Müller. Sources and technology for an atomic gravitational wave interferometric sensor. Gen. Relativ. Gravit., 43, 1905 (2011). (Cited on page 1.)

[26] G. Santarelli, C. Audoin, A. Makdissi, P. Laurent, G. J. Dick, and A. Clairon. Frequency Stability Degradation of an Oscillator Slaved to a Periodically Interrogated Atomic Resonator. Ultrason. Ferroelectr. Freq. Control IEEE Trans. On, 45, 887 (1998). (Cited on page 1.)

[27] D. DeMille, S. Sainis, J. Sage, T. Bergeman, S. Kotochigova, and E. Tiesinga. Enhanced Sensitivity to Variation of $\mathrm{m}_{\mathrm{e}} / \mathrm{m}_{\mathrm{p}}$ in Molecular Spectra. Phys. Rev. Lett., 100, 043202 (2008). (Cited on page 1.)

[28] N. Balakrishnan and A. Dalgarno. Chemistry at ultracold temperatures. Chem. Phys. Lett., 341, 652 (2001). (Cited on page 1.)

[29] A. André, D. DeMille, J. M. Doyle, M. D. Lukin, S. E. Maxwell, P. Rabl, R. J. Schoelkopf, and P. Zoller. A coherent all-electrical interface between polar molecules and mesoscopic superconducting resonators. Nat. Phys., 2, 636 (2006). (Cited on page 1.)

[30] C. F. Roos, P. Cren, D. Guéry Odelin, and J. Dalibard. Continuous loading of a non-dissipative atom trap. Europhys. Lett., 61, 187 (2003). (Cited on pages $2,41,46,50,51,69$ and 70.$)$

[31] A. E. Leanhardt, T. A. Pasquini, M. Saba, A. Schirotzek, Y. Shin, D. Kielpinski, D. E. Pritchard, and W. Ketterle. Cooling Bose-Einstein Condensates Below 500 Picokelvin. Science, 301, 1513 (2003). (Cited on page 3.)

[32] C. Salomon, J. Dalibard, W. D. Phillips, A. Clairon, and S. Guellati. Laser cooling of cesium atoms below $3 \mu \mathrm{K}$. Europhys. Lett., 12, 683 (1990). (Cited on page 3.) 
[33] D. J. Fixsen. The Temperature of the Cosmic Microwave Background. Astrophys. J., 707, 916 (2009). (Cited on page 3.)

[34] M. Stix. The Sun an Introduction. Astronomy and Astrophysics Library. Springer Berlin Heidelberg, Berlin, Heidelberg (2002). (Cited on page 3.)

[35] Bureau international des poids et mesures. Le système international d'unités: $(S I)=$ The international systems of units : (SI). Organisation intergouvernementale de la Convention du Mètre, Sèvres (2006). (Cited on page 4.)

[36] W. Demtröder. Experimentalphysik 1 Mechanik und Wärme. Springer, Berlin (2013). (Cited on page 4.)

[37] P. J. Mohr, B. N. Taylor, and D. B. Newell. CODATA recommended values of the fundamental physical constants: 2010. Rev. Mod. Phys., 84, 1527 (2012). (Cited on page 4.)

[38] W. Ketterle, D. S. Durfee, and D. M. Stamper-Kurn. Making, probing and understanding Bose-Einstein condensates. ArXiv Prepr. Cond-Mat9904034 (1999). (Cited on pages 5, 6 and 29.)

[39] A. Einstein. Quantentheorie des einatomigen idealen gases - zweite abhandlung. Sitzungsber. phys.-math. Kl. (1925). (Cited on page 6.)

[40] S. N. Bose. Plancks Gesetz und Lichtquantenhypothese. Z. phys, 26, 178 (1924). (Cited on page 6.)

[41] P. Kapitza. Viscosity of liquid helium below the $\lambda$-point. Nature, 141, 74 (1938). (Cited on page 6.)

[42] J. F. Allen and A. D. Misener. Flow of Liquid Helium II. Nature, 141, 75 (1938). (Cited on page 6.)

[43] F. London. The $\lambda$-Phenomenon of Liquid Helium and the Bose-Einstein Degeneracy. Nature, 141, 643 (1938). (Cited on page 6.)

[44] O. Penrose and L. Onsager. Bose-Einstein Condensation and Liquid Helium. Phys. Rev., 104, 576 (1956). (Cited on page 6.)

[45] A. L. Schawlow and C. H. Townes. Infrared and Optical Masers. Phys. Rev., 112, 1940 (1958). (Cited on page 6.)

[46] T. H. Maiman. Stimulated Optical Radiation in Ruby. Nature, 187, 493 (1960). (Cited on page 6.)

[47] D. E. Pritchard, E. L. Raab, V. Bagnato, C. E. Wieman, and R. N. Watts. Light Traps Using Spontaneous Forces. Phys. Rev. Lett., 57, 310 (1986). (Cited on page 6.) 
[48] E. L. Raab, M. Prentiss, A. Cable, S. Chu, and D. E. Pritchard. Trapping of Neutral Sodium Atoms with Radiation Pressure. Phys. Rev. Lett., 59, 2631 (1987). (Cited on page 6.)

[49] H. F. Hess. Evaporative cooling of magnetically trapped and compressed spin-polarized hydrogen. Phys. Rev. B, 34, 3476 (1986). (Cited on page 6.)

[50] T. Tommila. Cooling of Spin-Polarized Hydrogen Atoms Trapped in Magnetic-Field Minima. Europhys. Lett. EPL, 2, 789 (1986). (Cited on page 6.)

[51] N. Masuhara, J. M. Doyle, J. C. Sandberg, D. Kleppner, T. J. Greytak, H. F. Hess, and G. P. Kochanski. Evaporative Cooling of Spin-Polarized Atomic Hydrogen. Phys. Rev. Lett., 61, 935 (1988). (Cited on page 6.)

[52] N. Bogolubov. On the theory of superfluidity. J. Phys., 11, 23 (1947). (Cited on page 7.)

[53] E. P. Gross. Structure of a quantized vortex in boson systems. Il Nuovo Cimento, 20, 454 (1961). (Cited on page 7.)

[54] L. P. Pitaevskii. Vortex lines in an imperfect Bose gas. Sov. Phys. JETP, 13, 451 (1961). (Cited on page 7.)

[55] M. Falkenau, V. V. Volchkov, J. Rührig, A. Griesmaier, and T. Pfau. Continuous Loading of a Conservative Potential Trap from an Atomic Beam. Phys. Rev. Lett., 106, 163002 (2011). (Cited on page 7.)

[56] J. Doyle, B. Friedrich, J. Kim, and D. Patterson. Buffer-gas loading of atoms and molecules into a magnetic trap. Phys. Rev. A, 52, R2515 (1995). (Cited on page 7.)

[57] S. C. Doret, C. B. Connolly, W. Ketterle, and J. M. Doyle. Buffer-Gas Cooled Bose-Einstein Condensate. Phys. Rev. Lett., 103, 103005 (2009). (Cited on page 7.)

[58] R. Frisch. Experimenteller Nachweis des Einsteinschen Strahlungsrückstoßes. Z. Für Phys. Hadrons Nucl., 86, 42 (1933). (Cited on page 8.)

[59] D. A. Steck. Sodium D Line Data, available online at http://steck.us/alkalidata (revision 2.1.4) (2010). (Cited on pages 9 and 55.)

[60] M. Walhout, H. J. L. Megens, A. Witte, and S. L. Rolston. Magneto-optical trapping of metastable xenon: Isotope-shift measurements. Phys. Rev. A, 48, R879 (1993). (Cited on page 9.) 
[61] M. Okano, H. Hara, M. Muramatsu, K. Doi, S. Uetake, Y. Takasu, and Y. Takahashi. Simultaneous magneto-optical trapping of lithium and ytterbium atoms towards production of ultracold polar molecules. Appl. Phys. B, 98, 691 (2010). (Cited on page 9.)

[62] J. Prodan, A. Migdall, W. D. Phillips, I. So, H. Metcalf, and J. Dalibard. Stopping Atoms with Laser Light. Phys. Rev. Lett., 54, 992 (1985). (Cited on page 9.)

[63] Y. B. Ovchinnikov. A permanent Zeeman slower for Sr atomic clock. Eur. Phys. J. Spec. Top., 163, 95 (2008). (Cited on page 9.)

[64] V. I. Balykin, V. S. Letokhov, and V. I. Mishin. Cooling of sodium atoms by resonant laser emission. Zh Eksp Teor Fiz, 78, 1376 (1980). (Cited on page 9.)

[65] H. L. Bethlem, G. Berden, and G. Meijer. Decelerating neutral dipolar molecules. Phys. Rev. Lett., 83, 1558 (1999). (Cited on page 10.)

[66] H. L. Bethlem, G. Berden, F. M. H. Crompvoets, R. T. Jongma, A. J. A. van Roij, and G. Meijer. Electrostatic trapping of ammonia molecules. Nature, 406, 491 (2000). (Cited on page 11.)

[67] J. R. Bochinski, E. R. Hudson, H. J. Lewandowski, G. Meijer, and J. Ye. Phase Space Manipulation of Cold Free Radical OH Molecules. Phys. Rev. Lett., 91, 243001 (2003). (Cited on page 11.)

[68] S. Y. T. van de Meerakker, P. H. M. Smeets, N. Vanhaecke, R. T. Jongma, and G. Meijer. Deceleration and Electrostatic Trapping of $\mathrm{OH}$ Radicals. Phys. Rev. Lett., 94, 023004 (2005). (Cited on page 11.)

[69] S. Jung, E. Tiemann, and C. Lisdat. Cold atoms and molecules from fragmentation of decelerated $\mathrm{SO}_{2}$. Phys. Rev. A, 74, 040701 (2006). (Cited on page 11.)

[70] A. Marian, H. Haak, P. Geng, and G. Meijer. Slowing polar molecules using a wire Stark decelerator. Eur. Phys. J. D, 59, 179 (2010). (Cited on page 11.)

[71] S. A. Meek, H. L. Bethlem, H. Conrad, and G. Meijer. Trapping Molecules on a Chip in Traveling Potential Wells. Phys. Rev. Lett., 100, 153003 (2008). (Cited on page 11.)

[72] S. A. Meek, H. Conrad, and G. Meijer. Trapping Molecules on a Chip. Science, 324, 1699 (2009). (Cited on page 11.)

[73] S. A. Meek, H. Conrad, and G. Meijer. A Stark decelerator on a chip. New J. Phys., 11, 055024 (2009). (Cited on page 11.) 
[74] A. Aghajani-Talesh, M. Falkenau, V. V. Volchkov, L. E. Trafford, T. Pfau, and A. Griesmaier. Laser cooling of a magnetically guided ultracold atom beam. New J. Phys., 12, 065018 (2010). (Cited on page 12.)

[75] J. M. Sage, S. Sainis, T. Bergeman, and D. DeMille. Optical Production of Ultracold Polar Molecules. Phys. Rev. Lett., 94, 203001 (2005). (Cited on page 12.)

[76] E. R. Hudson. Method for producing ultracold molecular ions. Phys. Rev. A, 79, 032716 (2009). (Cited on page 13.)

[77] W. G. Rellergert, S. T. Sullivan, S. J. Schowalter, S. Kotochigova, K. Chen, and E. R. Hudson. Evidence for sympathetic vibrational cooling of translationally cold molecules. Nature, 495, 490 (2013). (Cited on page 13.)

[78] D. J. Cousins, S. N. Fisher, A. M. Guénault, R. P. Haley, I. E. Miller, G. R. Pickett, G. N. Plenderleith, P. Skyba, P. Y. A. Thibault, and M. G. Ward. An advanced dilution refrigerator designed for the new Lancaster microkelvin facility. J. Low Temp. Phys., 114, 547 (1999). (Cited on page 13.)

[79] F. Pobell. Matter and Methods at Low Temperatures. Springer Berlin Heidelberg, Berlin, Heidelberg (2007). (Cited on page 13.)

[80] S. Chaudhuri, S. Roy, and C. S. Unnikrishnan. Realization of an intense cold $\mathrm{Rb}$ atomic beam based on a two-dimensional magneto-optical trap: Experiments and comparison with simulations. Phys. Rev. A, 74, 023406 (2006). (Cited on pages 17 and 36.)

[81] R. Hodajerdi. Aufbau einer zweidimensionalen magneto-optischen Falle mit hohem Fluss. Diploma Thesis, Gottfried Wilhelm Leibniz Universität Hannover, Hannover (2009). (Cited on page 20.)

[82] S. Jöllenbeck. Eine Quelle Bose-Einstein-kondensierter Ensembles auf Basis eines mesoskopischen Atomchips. PhD Thesis, Gottfried Wilhelm Leibniz Universität Hannover, Hannover (20.07.2012). (Cited on pages 20, 31 and 33.)

[83] M. Gilowski, C. Schubert, M. Zaiser, W. Herr, T. Wübbena, T. Wendrich, T. Müller, E. Rasel, and W. Ertmer. Narrow bandwidth interference filterstabilized diode laser systems for the manipulation of neutral atoms. Opt. Commun., 280, 443 (2007). (Cited on page 20.)

[84] U. Schünemann, H. Engler, R. Grimm, M. Weidemüller, and M. Zielonkowski. Simple scheme for tunable frequency offset locking of two lasers. Rev. Sci. Instrum., 70, 242 (1999). (Cited on page 20.) 
[85] J. Mahnke. Eine magneto-optische Falle auf Basis eines mesoskopischen Atomchips. Diploma Thesis, Gottfried Wilhelm Leibniz Universität Hannover, Hannover (23.06.2010). (Cited on page 25.)

[86] I. Geisel. Präparation eines magnetisch geführten Strahls ultrakalter Atome. Master's Thesis, Gottfried Wilhelm Leibniz Universität Hannover, Hannover (27.07.2011). (Cited on page 25.)

[87] I. Geisel, K. Cordes, J. Mahnke, S. Jöllenbeck, J. Ostermann, J. Arlt, W. Ertmer, and C. Klempt. Evolutionary optimization of an experimental apparatus. Appl. Phys. Lett., 102, 214105 (2013). (Cited on page 26.)

[88] R. Storn and K. Price. Differential evolution - A simple and efficient heuristic for global optimization over continuous spaces. J. Glob. Optim., 11, 341 (1997). (Cited on page 26.)

[89] K. V. Price, R. M. Storn, and J. A. Lampinen. Differential Evolution - A practical approach to global optimization. Natural computing series. Springer, Berlin ; New York (2005). (Cited on page 26.)

[90] K.-J. Kügler, W. Paul, and U. Trinks. A magnetic storage ring for neutrons. Phys. Lett. B, 72, 422 (1978). (Cited on page 27.)

[91] H. J. Metcalf. Laser cooling and trapping. Graduate texts in contemporary physics. Springer, New York (1999). (Cited on page 27.)

[92] J. D. Weinstein and K. G. Libbrecht. Microscopic magnetic traps for neutral atoms. Phys. Rev. A, 52, 4004 (1995). (Cited on page 28.)

[93] R. Folman, P. Krüger, D. Cassettari, B. Hessmo, T. Maier, and J. Schmiedmayer. Controlling Cold Atoms using Nanofabricated Surfaces: Atom Chips. Phys. Rev. Lett., 84, 4749 (2000). (Cited on page 28.)

[94] W. Demtröder. Experimentalphysik 3 Atome, Moleküle und Festkörper. Springer-Lehrbuch. Springer Berlin Heidelberg, Berlin, Heidelberg (2010). (Cited on page 28.)

[95] J. Reichel, W. Hänsel, P. Hommelhoff, and T. Hänsch. Applications of integrated magnetic microtraps. Appl. Phys. B, 72, 81 (2001). (Cited on page 29.)

[96] J. Reichel and V. Vuletić. Atom Chips. Wiley-VCH, Weinheim, Germany (2011). (Cited on page 29.) 
[97] D. Cassettari, A. Chenet, R. Folman, A. Haase, B. Hessmo, P. Krüger, T. Maier, S. Schneider, T. Calarco, and J. Schmiedmayer. Micromanipulation of neutral atoms with nanofabricated structures. Appl. Phys. B, 70, 721 (2000). (Cited on pages 30 and 32.)

[98] J. Fortágh and C. Zimmermann. Magnetic microtraps for ultracold atoms. Rev. Mod. Phys., 79, 235 (2007). (Cited on pages 31 and 32.)

[99] A. Günther, M. Kemmler, S. Kraft, C. J. Vale, C. Zimmermann, and J. Fortágh. Combined chips for atom optics. Phys. Rev. A, 71 (2005). (Cited on page 31.)

[100] S. Wildermuth, P. Krüger, C. Becker, M. Brajdic, S. Haupt, A. Kasper, R. Folman, and J. Schmiedmayer. Optimized magneto-optical trap for experiments with ultracold atoms near surfaces. Phys. Rev. A, 69, 030901(R) (2004). (Cited on page 31.)

[101] M. Hyodo, K. Nakayama, M. Watanabe, and R. Ohmukai. Mirror magnetooptical trap exploiting hexapole-compensated magnetic field. Phys. Rev. A, 76, 013419 (2007). (Cited on page 31.)

[102] S. Jöllenbeck, J. Mahnke, R. Randoll, W. Ertmer, J. Arlt, and C. Klempt. Hexapole-compensated magneto-optical trap on a mesoscopic atom chip. Phys. Rev. A, 83, 043406 (2011). (Cited on pages 31, 33 and 71.)

[103] J. Thywissen, M. Olshanii, G. Zabow, M. Drndić, K. Johnson, R. Westervelt, and M. Prentiss. Microfabricated magnetic waveguides for neutral atoms. Eur. Phys. J. -At. Mol. Opt. Plasma Phys., 7, 361 (1999). (Cited on page 32.)

[104] W. Hänsel, J. Reichel, P. Hommelhoff, and T. W. Hänsch. Magnetic Conveyor Belt for Transporting and Merging Trapped Atom Clouds. Phys. Rev. Lett., 86, 608 (2001). (Cited on page 33.)

[105] M. Prentiss, A. Cable, J. E. Bjorkholm, S. Chu, E. L. Raab, and D. E. Pritchard. Atomic-density-dependent losses in an optical trap. Opt. Lett., 13, 452 (1988). (Cited on page 33.)

[106] E. Majorana. Atomi orientati in campo magnetico variabile. Il Nuovo Cimento, 9, 43 (1932). (Cited on page 34.)

[107] Y. B. Ovchinnikov. Compact magneto-optical sources of slow atoms. Opt. Commun., 249, 473 (2005). (Cited on page 36.)

[108] J. Reichel, W. Hänsel, and T. W. Hänsch. Atomic Micromanipulation with Magnetic Surface Traps. Phys. Rev. Lett., 83, 3398 (1999). (Cited on page 36.) 
[109] W. Petrich, M. H. Anderson, J. R. Ensher, and E. A. Cornell. Behavior of atoms in a compressed magneto-optical trap. J. Opt. Soc. Am. B, 11, 1332 (1994). (Cited on page 37.)

[110] J. Dalibard and C. Cohen-Tannoudji. Laser cooling below the Doppler limit by polarization gradients: simple theoretical models. J. Opt. Soc. Am. B, 6, 2023 (1989). (Cited on page 37.)

[111] J. Mahnke, I. Kruse, A. Hüper, S. Jöllenbeck, W. Ertmer, J. Arlt, and C. Klempt. A continuously pumped reservoir of ultracold atoms. J. Phys. B At. Mol. Opt. Phys., 48, 165301 (2015). (Cited on page 43.)

[112] D. A. Steck. Cesium D Line Data, available online at http://steck.us/alkalidata (revision 2.1.4) (2010). (Cited on page 55.)

[113] D. A. Steck. Rubidium 85 D Line Data, available online at http://steck.us/alkalidata (revision 2.1.6) (2013). (Cited on page 55.)

[114] D. A. Steck. Rubidium 87 D Line Data, available online at http://steck.us/alkalidata (revision 2.1.4) (2010). (Cited on pages 55, 59 and 75.)

[115] S. Ezekiel. Investigation of a cesium Raman time/frequency standard. Technical report, DTIC Document (1991). (Cited on page 56.)

[116] C. J. Myatt, N. R. Newbury, and C. E. Wieman. Simplified atom trap by using direct microwave modulation of a diode laser. Opt. Lett., 18, 649 (1993). (Cited on page 56.)

[117] G. Stern, B. Allard, M. Robert-de Saint-Vincent, J.-P. Brantut, B. Battelier, T. Bourdel, and P. Bouyer. Frequency doubled $1534 \mathrm{~nm}$ laser system for potassium laser cooling. Appl. Opt., 49, 3092 (2010). (Cited on page 56.)

[118] A. Waxman, M. Givon, G. Aviv, D. Groswasser, and R. Folman. Modulation enhancement of a laser diode in an external cavity. Appl. Phys. B, 95, 301 (2009). (Cited on page 56.)

[119] J. Ringot, Y. Lecoq, J. C. Garreau, and P. Szriftgiser. Generation of phasecoherent laser beams for Raman spectroscopy and cooling by direct current modulation of a diode laser. Eur. Phys. J. D, 7, 285 (1999). (Cited on page 56.)

[120] R. Kowalski, S. Root, S. D. Gensemer, and P. L. Gould. A frequencymodulated injection-locked diode laser for two-frequency generation. Rev. Sci. Instrum., 72, 2532 (2001). (Cited on page 56.) 
[121] S. Kulas. Lasersysteme zur Präparation und Manipulation ultrakalter Ensembles für die Atominterferometrie. Master's Thesis, Leibniz Universität Hannover, Hannover (2011). (Cited on pages 56 and 62.)

[122] J. Mahnke, S. Kulas, I. Geisel, S. Jöllenbeck, W. Ertmer, and C. Klempt. Microwave sidebands for laser cooling by direct modulation of a tapered amplifier. Rev. Sci. Instrum., 84, 063110 (2013). (Cited on page 56.)

[123] L. Goldberg, D. Mehuys, M. R. Surette, and D. C. Hall. High-power, neardiffraction-limited large-area traveling-wave semiconductor amplifiers. IEEE J. Quantum Electron., 29, 2028 (1993). (Cited on page 56.)

[124] E. Gehrig and O. Hess. Spatio-Temporal Dynamics and Quantum Fluctuations in Semiconductor Lasers, volume 189 of Springer Tracts in Modern Physics. Springer, Berlin, Heidelberg (2003). (Cited on pages 56 and 57.)

[125] Eagleyard-Homepage. http://www.eagleyard.com/en/products/taperedlaser-amplifier/ (2015). (Cited on page 57.)

[126] J. Arlt. Experiments on Bose-Einstein Condensation. PhD thesis, University of Oxford, Oxford (2000). (Cited on page 58.)

[127] S. Chu, J. E. Bjorkholm, A. Ashkin, and A. Cable. Experimental Observation of Optically Trapped Atoms. Phys. Rev. Lett., 57, 314 (1986). (Cited on page 66.)

[128] J. C. Zappala, K. Bailey, Z.-T. Lu, T. P. O'Connor, and W. Jiang. Note: Efficient generation of optical sidebands at $\mathrm{GHz}$ with a high-power tapered amplifier. Rev. Sci. Instrum., 85, 046104 (2014). (Cited on page 67.)

[129] D. M. Harber, J. M. McGuirk, J. M. Obrecht, and E. A. Cornell. Thermally Induced Losses in Ultra-Cold Atoms Magnetically Trapped Near RoomTemperature Surfaces. J. Low Temp. Phys., 133, 229 (2003). (Cited on page 71.)

[130] G. Reinaudi, T. Lahaye, A. Couvert, Z. Wang, and D. Guéry Odelin. Evaporation of an atomic beam on a material surface. Phys. Rev. A, 73, 035402 (2006). (Cited on page 71.)

[131] A. P. Chikkatur. A Continuous Source of Bose-Einstein Condensed Atoms. Science, 296, 2193 (2002). (Cited on page 71.)

[132] N. P. Robins, C. Figl, M. Jeppesen, G. R. Dennis, and J. D. Close. A pumped atom laser. Nat. Phys., 4, 731 (2008). (Cited on page 71.) 
[133] M.-O. Mewes, M. R. Andrews, D. M. Kurn, D. S. Durfee, C. G. Townsend, and W. Ketterle. Output Coupler for Bose-Einstein Condensed Atoms. Phys. Rev. Lett., 78, 582 (1997). (Cited on page 71.) 


\section{Publications}

[P1] S. Jöllenbeck, J. Mahnke, R. Randoll, W. Ertmer, J. Arlt, and C. Klempt. Hexapole-compensated magneto-optical trap on a mesoscopic atom chip. Phys. Rev. A, 83, 043406 (2011).

[P2] I. Geisel, K. Cordes, J. Mahnke, S. Jöllenbeck, J. Ostermann, J. Arlt, W. Ertmer, and C. Klempt. Evolutionary optimization of an experimental apparatus. Appl. Phys. Lett., 102, 214105 (2013).

[P3] J. Mahnke, S. Kulas, I. Geisel, S. Jöllenbeck, W. Ertmer, and C. Klempt. Microwave sidebands for laser cooling by direct modulation of a tapered amplifier. Rev. Sci. Instrum., 84, 063110 (2013).

[P4] J. Mahnke, I. Kruse, A. Hüper, S. Jöllenbeck, W. Ertmer, J. Arlt, and C. Klempt A continuously pumped reservoir of ultracold atoms. J. Phys. B At. Mol. Opt. Phys., 48, 165301 (2015). 



\section{Lebenslauf}

\section{Persönliche Daten}

Name

Geburtsdatum/-ort

Familienstand

\section{Promotions-Studium}

seit 2010

\section{Studium}

$06 / 2009-06 / 2010$

$2003-2010$
Jan Mahnke

29.05.1984 in Hannover, Deutschland

ledig

Doktorarbeit am Institut für Quantenoptik der Leibniz Universität Hannover

Diplomarbeit am Institut für Quantenoptik der Leibniz Universität Hannover mit dem Thema "Eine magneto-optische Falle auf Basis eines mesoskopischen Atomchips"

Studium der Physik an der Leibniz Universität Hannover

\section{Schulabschluss}

Abitur an der Ricarda-Huch-Schule in Hannover 



\section{Danksagung}

An dieser Stelle möchte ich mich ganz herzlich bei allen Menschen bedanken, die zum Gelingen der Arbeit beigetragen haben, die mich mit Rat und Tat unterstützt haben oder einfach meine Doktorandenzeit bereichert haben!

Herrn Prof. Wolfgang Ertmer danke ich für die Betreuung meiner Arbeit, für das gute Arbeitsklima und die hervorragenden Forschungsbedingungen, die er am Institut geschaffen hat. PD. Dr. Carsten Klempt möchte ich für die kollegiale Leitung des Experiments danken, für die vielen fruchtbaren Diskussionen und für die Hilfe in allen möglichen vorhergesehenen und unvorhergesehenen Situationen. Danken möchte ich auch Prof. Luis Santos für die Leitung der Research Training Group 1729 „Fundamentals and applications of ultra-cold matter“. Diese hat mir viele spannende Tagungen, lehrreiche Vorlesungen sowie einen regen Austausch mit anderen Doktoranden auf dem Gebiet ermöglicht und nicht zuletzt einen Großteil der Finanzierung erbracht. Im Rahmen der RTG konnte ich auch an einem Mentoring Programm teilnehmen, bei dem mir Jun.-Prof. Teimuraz Vekua hilfreich zur Seite stand. Auch hierfür möchte ich mich bedanken.

Besonders das Institut für Quantenoptik lebt von vielen kurzweiligen Mitstreitern und einem sehr familiären und hilfsbereiten Klima, weshalb sich mein Dank auch an die Kollegen richtet. Danken möchte ich Stefan Jöllenbeck, der das Experiment aufgebaut und mich sehr herzlich aufgenommen hat. Ilka Kruse, mit der ich den Großteil meiner Doktorandenzeit am Experiment teilen durfte, möchte ich für den Enthusiasmus und die stetige Unterstützung danken. Ein herzlicher Dank geht auch an Andreas Hüper und Dr. Jiao Geng, die das Team auf vielfältige Weise bereichert haben. Bedanken möchte ich mich auch bei Dr. Bernd Lücke, Dr. Jan Peise und Karsten Lange für die freundschaftliche Atmosphäre in der Gruppe und die vielen, schönen Momente.

Ein Dankeschön geht auch an die „Struktur“ des Instituts: Das SekretärinnenTeam: Gunhild Faber, Elke Hünitzsch, Natalie Koberstein, Katrin Pfennig und Bettina Zander. Ohne sie wäre ich wohl das ein oder andere Mal verloren gewesen. Eine große Hilfe war auch das Werkstatt-Team um Fritz Witzlau, das die Fertigung des kniffligen Atomchips mit Bravour gemeistert hat.

Ein Dank geht sicher auch an meine Freunde. Hannes und Nina für so vieles, aber in letzter Zeit wohl besonders für Care-Pakete und offene Ohren. Dirk, Heiko und Torben danke ich für die lange Freundschaft, das Salz in der Suppe.

Ein besonderer Dank geht natürlich auch an meine Eltern Hanne und Peter für ihre ungebrochene Unterstützung und dafür, dass sie meine Eltern sind. Danke auch an Birte für das Ertragen der entbehrungsreichen Zeiten, die vielen schönen Momente und die Liebe. Danke! 